\title{
Taxonomic revision of the genus Arsipoda Erichson, 1842 (Coleoptera, Chrysomelidae) in New Caledonia
}

\author{
Paola D’ALESSANDRO ${ }^{1, *}$, Allan SAMUELSON ${ }^{2} \&$ Maurizio BIONDI $^{3}$ \\ ${ }^{1,3}$ Department of Health, Life and Environmenal Sciences, University of L'Aquila, \\ 67100 Coppito-L'Aquila, Italy. \\ ${ }^{2}$ J. Linsley Gressitt Center for Research in Entomology, Bishop Museum, \\ 1525 Bernice Street, Honolulu, HI 96817, U.S.A. \\ *Corresponding author: paola.dalessandro@univaq.it \\ ${ }^{2}$ Email: iiwi@hawaii.rr.com \\ ${ }^{3}$ Email: maurizio.biondi@univaq.it \\ ${ }^{1}$ urn:1sid:zoobank.org:author:7B73D5BC-AA91-4D3D-AADF-641DA1DD42DD \\ ${ }^{2}$ urn:1sid:zoobank.org:author:4471B588-416D-4136-B350-9857FB969E3B \\ ${ }^{3}$ urn:lsid:zoobank.org:author:E7B2A93B-540A-4AF4-9645-3318CB154C84
}

\begin{abstract}
A taxonomic revision of the New Caledonian species of Arsipoda Erichson, 1842 is provided. This group includes 21 species, of which 14 are new to science: Arsipoda atra sp. nov., A. communis sp. nov., A. doboszi sp. nov., A. elongata sp. nov., A. gomezzuritai sp. nov., A. gressitti sp. nov., A. longifrons sp. nov., A. montana sp. nov., A. paniensis sp. nov., A. povilaensis sp. nov., A. punctata sp. nov., A. rutai sp. nov., A. transversa sp. nov. and $A$. wanati sp. nov. A key for the identification, with figures of habitus, main diagnostic characters, and genitalia is supplied. The range of host plants for these species is extraordinarily broad, and a significant number of them feed on pollen. Crepicnema Scherer, 1969, close to Arsipoda, is also investigated and maintained as a separate genus, and the following synonymies and combination are proposed: Crepicnema parvula (Jacoby, 1885) comb. nov. $=$ Chaetocnema tenimberensis Jacoby, 1894 syn. nov., = Arsipoda salomonensis Bryant, 1941 syn. nov. The phylogenetic analysis, including also A. bifrons Erichson, 1842 and Crepicnema, confirms some trends in distribution patterns of the endemic New Caledonian fauna, and highlights the necessity of further studies to clarify the relationships between Arsipoda and related genera.
\end{abstract}

Key words. Dichotomous key, new combinations, new species, Pacific Island, pollen feeding.

D'Alessandro P., Samuelson A. \& Biondi M. 2016. Taxonomic revision of the genus Arsipoda Erichson, 1842 (Coleoptera, Chrysomelidae) in New Caledonia. European Journal of Taxonomy 230: 1-61. http://dx.doi. org/10.5852/ejt.2016.230

\section{Introduction}

New Caledonia as the setting for this study is noted as one of Earth's Biodiversity Hotspots (AA.VV. 2011; Kier et al. 2009; Lowry et al. 2004; Mittermeier et al. 2004; Myers et al. 2000; Myers 2003; 
Possingham \& Wilson 2005). This area is characterized as a model for isolated islands, marked by a high degree of faunal disharmony (Gillespie \& Roderick 2002; Gómez-Zurita 2011), with relatively few established genera that have had more opportunities to radiate and fill available niches, generating a high degree of microendemism (D'Alessandro et al. 2014; Gasc et al. 2013; Grandcolas et al. 2008; Nattier et al. 2013). The origin of New Caledonian biota is controversial, because there is evidence for both an ancient Gondwanan origin - implying the persistence of at least some original Gondwanan land masses now forming New Caledonian territory -, and a more recent, late Eocenic or Oligocenic origin that is based on the hypotheses of a total submersion of the territory during the Eocene (Espeland \& Murienne 2011; Jolivet \& Verma 2010; Papadopoulou et al. 2013). The first hypothesis is compatible with the presence of some supposed Gondwanan relicts explained by ancient vicariance events and subsequent diversification; the second one implies only recent dispersal events at the origin of the biota and can justify the disharmonic composition of many New Caledonian taxa compared with the fauna of other Gondwanan landmasses (Gillespie \& Roderick 2002; Gómez-Zurita 2011), as well as the high degree of neoendemism (Nattier et al. 2012, 2013). The New Caledonian Chrysomelidae fauna has been investigated by Jolivet \& Verma $(2008,2009)$. It includes 138 described species in 41 genera with an endemism rate of $83.2 \%$ at the species level (Jolivet \& Verma 2009). However, as also Jolivet \& Verma $(2008,2009)$ pointed out, New Caledonia and the neighbouring south-eastern Pacific islands have been only partly explored. Indeed, other recent papers (Beenen 2008, 2013; Gómez-Zurita \& Cardoso 2013; Gómez-Zurita et al. 2010; Samuelson 2010) increased the number of species for different taxa.

The Chrysomelidae is one of the largest phytophagous insect families and includes approximately 37 000-40 000 species (Biondi \& D'Alessandro 2012). The monophyletic tribe Alticini is closely related to the tribe Galerucini, both contained within in the subfamily Galerucinae (Bouchard et al. 2011). This paper, in particular, deals with Arsipoda Erichson 1842, a genus now recognized as one of the notable examples of chrysomelid radiation in New Caledonia. It is one of the 11 flea beetle genera (Chrysomelidae, Galerucinae, Alticini) occurring in New Caledonia (Jolivet \& Verma 2009). Currently, about 75 species are included in this genus, which is centered in Australia and extended to New Caledonia, New Guinea with associated smaller islands, and the Solomon Islands (Heikertinger \& Csiki 1940; Gómez-Zurita et al. 2010; Mohamedsaid 2004; Samuelson 1973; Scherer 1969; Seeno \& Wilcox 1982). The diagnostic characters and phylogenetic affinities of Arsipoda are not stable in the literature (see Gómez-Zurita et al. 2010 for a summary); the examination of type material attributed to this genus and kept at the British Museum of Natural History (London) and Bishop Museum (Honolulu) actually revealed that many species differ greatly from each other and from the type species A. bifrons Erichson, 1842, thus, in our opinion, some of them should be placed in different genera. Anyway, a complete revision of this genus is beyond of the aim of this paper that, instead, wants to focus on the group of species occurring in New Caledonia that we consider here as belonging to Arsipoda. The New Caledonian Arsipoda were studied for the first time by one of us (Samuelson 1973), who described A. agalma Samuelson, 1973, A. isola Samuelson, 1973, A. shirleyae Samuelson, 1973, A. yiambiae Samuelson, 1973 and A. evax Samuelson, 1973. Later, Gómez-Zurita et al. (2010) added two new species, A. geographica GómezZurita, 2010 and $A$. rostrata Gómez-Zurita, 2010. All of them are from the main island of Grande Terre (only A. evax has been collected on Belep Island), none is recorded either from the Loyalty Islands or the Isle of Pines. In this paper, we provide new distributional data on the known species, the description of 14 new species from the Grande Terre, and a dichotomous key to all the species. We also report ecological information derived from label data, and investigate about pollen consumption by looking for the presence of pollen grains in the gut of the examined specimens. We recognize the monobasic Oriental genus Crepicnema Scherer, 1969 as being closely allied to certain species of Arsipoda. Seeno and Wilcox (1982) also put the two genera close to each other, and some authors even recently referred to Crepicnema tenimberensis (Jacoby, 1894) as Arsipoda tenimberensis (Jacoby, 1894) (Kimoto et al. 1984; Gómez-Zurita et al. 2010). Thus we analysed the taxonomic position of Crepicnema and include its results in our analysis below. 


\section{Material and methods}

Material examined consisted of more than 1550 dried and pinned specimens preserved in the institutions listed below. Specimens were examined, measured and dissected using Wild MZ12.5 and Leica M205C binocular microscopes. The dissections were aimed at the extraction of the median lobe of aedeagus and the spermatheca, and at the recovery of pollen grains inside the gut. Photomicrographs were taken with a Leica DFC500 camera and the Auto-Montage Pro 2006 software (license number: 15224*syn2459*153a2112_maurizio_266836). Scanning electron micrographs were taken with a HITACHI TM-1000. Geographical coordinates of the localities were reported in the format used in the respective labels; coordinates and geographical information included in square brackets and reported in decimal degrees (DD-WGS84 format) were added by the authors using the website of Google Earth. The terminology used follows Döberl (1986), Furth \& Suzuki (1994) and Suzuki (1988) for the spermatheca (Fig. 11E); Furth \& Suzuki (1998) for the metafemoral spring (Fig. 6F); for the median lobe of aedeagus see Fig. 10E. The cladistic analysis of the morphological dataset was performed with aid of the program TNT v1.1 licensed to the Willi Hennig Society using the traditional search algorithm ('Wagner trees'), non additive characters and implied weighting method. According to Goloboff et al. (2008a, 2008b) and Goloboff (2013), character weighting by implied weighting method was used in our analysis because jackknife frequencies and other resampling measures are, for morphological data, clearly improved when weighting against homoplasy. The $\mathrm{K}$ value (constant of concavity) was chosen by us with the aid of the script 'Implied_w' proposed by Mirande (2009). The morphological dataset used for the analysis includes 34 characters, of which 33 bi-state and 1 tri-state. Eighteen characters were derived from the external anatomy and 16 from the genitalic structures (median lobe of aedeagus and spermatheca). We analyzed 23 taxa, i.e., the 21 New Caledonian Arsipoda species, with Arsipoda bifrons Erichson, 1842 and Crepicnema parvula (Jacoby, 1885), treated as outgroups.

\section{Abbreviations}

\section{Morphology}

LA = numerical sequence proportional to length of each antennomere

LAED $=$ length of median lobe of aedeagus

LAN $=$ length of antennae

LB = total length of body

LE $\quad=$ length of elytra

$\mathrm{LP} \quad=$ length of pronotum

LSPc $=$ length of spermathecal capsule

und. = undetectable (damaged body parts were not measured and are reported as undetectable)

$\mathrm{WE} \quad=$ width of elytra

WP $\quad$ width of pronotum

\section{Collections and depositories}

BAQ = collection of M. Biondi, Department of Life, Health and Environmental Sciences, University of L'Aquila, Italy

$\mathrm{BMNH}=$ The Natural History Museum, London, U.K.

BPBM = Bernice Pauahi Bishop Museum, Honolulu, Hawaii, U.S.A.

$\mathrm{MNHN}=$ Museum national d'Histoire naturelle, Paris, France

MNHW $=$ Museum of Natural History, University of Wroclaw, Poland

MSNG $=$ Museo Civico di Storia Naturale 'Giacomo Doria', Genova, Italy

NZAC $=$ NZ Landcare Research, Auckland, New Zealand

USMB $=$ Upper Silesian Museum, Bytom, Poland 


\title{
Results
}

Taxonomic hierarchy (based on Bouchard et al. 2011; Slipinski et al. 2011 and Zhang 2011).

\author{
Class Hexapoda Latreille, 1825 \\ Order Coleoptera Linnaeus, 1758 \\ Suborder Polyphaga Emery, 1886 \\ Superfamily Chrysomeloidea Latreille, 1802 \\ Family Chrysomelidae Latreille, 1802 \\ Subfamily Galerucinae Latreille, 1802 \\ Tribe Alticini Newman, 1834
}

Genus Arsipoda Erichson, 1842

Arsipoda Erichson, 1842: 235.

Eratosthenes Clark, 1864: 261. (synonymized by Chapuis 1875)

\section{Key to New Caledonian species of Arsipoda}

1. Frontal grooves very deeply impressed and elongate, generally continued to postantennal region (Fig. 4A). Pronotal punctuation on disc formed by very large and strongly impressed punctures, but almost absent behind antebasal sulcus (Fig. 4A). Pronotum slightly convex. Median lobe of aedeagus in Figs 8A-B. Spermatheca as in Fig. 11A... Arsipoda agalma Samuelson, 1973

- Frontal grooves from weakly to strongly impressed but not clearly continued to postantennal region (Figs 4B-F, 5A-F). Pronotal punctuation from very weakly to distinctly impressed, but formed by smaller punctures, generally including base of pronotum behind antebasal sulcus (Figs 4B-F, 5A-F). Pronotum distinctly convex

2. Antennae clearly bicolored with antennomeres 1-4 yellow and 5-11 dark brown (Fig. 3A). Maxillary and labial palpi blackish, distinctly darker than other mouth parts. Body yellowish. Pronotal punctuation very weakly impressed (Fig. 5E). Median lobe of aedeagus in ventral view laterally parallel and apically dominantly and regularly rounded (Fig. 10B). Spermatheca as in Fig. 11Q.

A. wanati sp. nov.

- Antennae largely yellowish or brownish, often gradually and slightly darkened towards distal part (Figs 1B-I, 2A-I, 3B-C). Maxillary and labial palpi similar in color to other mouth parts. Body from yellowish to blackish. Pronotal punctuation more distinctly impressed (Figs 4B-F, 5A-D, 5F). Median lobe of aedeagus in ventral view laterally sinuate or tapered, rarely subparallel; apically acute, subtriangular or with an evident median small tooth (only in A. povilaensis sp. nov. broadly rounded, but different in lateral view) (Figs 8C-I, 9A-I, 10A, C-D).

3. Pronotal punctuation sparsely and unevenly distributed (Fig. 4C). Median lobe of aedeagus in ventral view distinctly enlarged at basal fourth and clearly tapered from base to apex; in lateral view dorsally bisinuate (Fig. 8E). Spermatheca with elongate basal part and short distal appendix (Fig. 11D).

A. doboszi sp. nov.

- Pronotal punctuation densely and evenly distributed (Figs 4B, D-F, 5A-D, F). Median lobe of aedeagus in ventral view not enlarged at basal fourth and dorsally not bisinuate in lateral view (Figs 8C-D, F-I, 9A-I, 10A, C-D). Spermatheca generally with basal part subglobose, otherwise distal appendix distinctly more elongate (Fig. 11B-C, E-P, R-S).

4. Elytra with wide and flat interstriae, and punctuation formed by small punctures (Fig. 6A). Dorsal integuments black without metallic reflection (Fig. 1B). Median lobe of aedeagus in ventral view apically with a narrow, elongate and flat extension (Fig. 8C). Spermatheca with single-coiled ductus (Fig. 11B)..... A. atra sp. nov. 
- Elytra with narrower and subconvex interstriae, and punctuation generally formed by larger punctures (Fig. 6B-C). Dorsal integuments yellowish, bronze, dark green or blackish, generally with distinct metallic reflection (Figs 1C, E-I, 2A-I, 3B-C). Median lobe of aedeagus (Figs 8D, F-I, 9A-I, $10 \mathrm{~A}, \mathrm{C}-\mathrm{D})$ in ventral view apically without elongate and flat extension. Spermatheca with uncoiled ductus (Fig. 11C, E-P, R-S) .

5. Body smaller (LB: $\widehat{\partial} \leq 1.90$ and $q \leq 1.84 \mathrm{~mm}$ ). Dorsal integuments blackish, with evident greenish metallic reflection (Fig. 1F). Elytra comparatively shorter (WE/LE: $\delta \geq 0.70$ and $q$ generally $\geq$ 0.69). Median lobe of aedeagus shorter (LAED $\leq 0.71 \mathrm{~mm}$ ) (Fig. 8G). Spermatheca with thick ductus (Fig. 11F). A. evax Samuelson, 1973

- Body larger (LB: ô generally $>1.90$ and $\uparrow>1.84 \mathrm{~mm}$ ). Dorsal integuments variable in color from yellowish to blackish, generally with metallic reflection (Figs 1C, E, G-I, 2A-I, 3B-C). Elytra comparatively longer (WE/LE: $\lambda<0.70$ and $q$ generally $<0.69$ ). Median lobe of aedeagus always longer (LAED $>0.71 \mathrm{~mm}$ ). Spermatheca with thinner ductus (with the exception of $A$. povilaensis sp. nov. and A. yiambiae; but these species are clearly different in size, sculpture and color from $A$. evax) (Fig. 11C, E, G-P, R-S) .

6. Pronotal punctuation with large and strongly impressed punctures, in some cases differently sized (Figs 4D, 5B-C).

- Pronotal punctuation with more weakly impressed and always evenly sized punctures (Figs 4B, E-F, $5 \mathrm{~A}, \mathrm{D}, \mathrm{F})$.....

7. Dorsal integuments blackish with evident green metallic reflection (Fig. 2G). Median lobe of aedeagus in ventral view more slender, laterally tapered towards apex (Fig. 9H); ventral sulcus narrow and moderately but distinctly impressed from base to apex. Spermatheca as in Fig. 11N .....

A. rutai sp. nov.

- Dorsal integuments yellowish, slightly metallic or light brown with evident bronze reflection (Figs 1I, 2E). Median lobe of aedeagus in ventral view more thickset, laterally parallel or tapered toward base (Fig. 9A, F).

8. Pronotum distinctly transverse (WP/LP: $\widehat{O} \geq 1.64$ ), with evenly sized punctuation (Fig. 5B). Dorsal integuments yellowish, slightly metallic (Fig. 2E). Median lobe of aedeagus (Fig. 9F) short (LAED $\leq$ $0.79 \mathrm{~mm}$; LE/LAED $\geq 1.88$ ) in ventral view laterally tapered toward base; ventral sulcus complete, shallow, narrowed at basal third. Female unknown.....

A. punctata sp. nov.

- Pronotum weakly transverse (WP/LP: $\hat{\delta}<1.64$ ) with unevenly sized punctuation (Fig. 4D). Dorsal integuments light brown with evident bronze reflection (Fig. 1I). Median lobe of aedeagus (Fig. 9A) more elongate (LAED $>0.79 \mathrm{~mm}$; LE/LAED $<1.88$ ) in ventral view, laterally subparallel; ventral sulcus incomplete, wide and open in apical part, then partially closed. Spermatheca as in Fig. 11I. A. isola Samuelson, 1973

9. Antennae distinctly elongate (LAN/LB: $\widehat{\AA} \geq 0.70$, $q$ generally $\geq 0.60$ ) (Figs $1 \mathrm{E}, 2 \mathrm{~A}, \mathrm{~F}, 3 \mathrm{C}) \ldots \ldots . .10$

- Antennae shorter (LAN/LB: $\hat{\delta}<0.70,9<0.60)$ (Figs 1C, G-H, 2B-D, H-I, 3B) ....................... 13

10. Elytra more elongate (WE/LE: $\delta$ and $q \leq 0.60$ ) (Fig. 1E). Median lobe of aedeagus slender (Fig. 8F). Basal part of spermatheca globose (Fig. 11E)

A. elongata sp. nov.

- Elytra shorter (WE/LE: $\delta$ and $q>0.60$ ). Median lobe of aedeagus more thickset (Figs 9B, 9G, 10D). Basal part of spermatheca more elongate or olive shaped (Fig. 11J, M, S) .

11. Median lobe of aedeagus (Fig. 10D) in ventral view laterally narrower in middle part; apical part widely lanceolate; ventral sulcus wide and deeply impressed from base to subapical part; in lateral view, median lobe rather straight. Spermathecal capsule (Fig. 11S) slender and generally curved 
- Median lobe of aedeagus (Fig. 9B, G) in ventral view laterally subparallel or tapered but not medially narrow; apical part not lanceolate in distal half; ventral sulcus obliterate or narrower and more weakly impressed in basal half; in lateral view, median lobe always more or less curved. Spermathecal capsule thickset and straight (Fig. 11J, M)

12. Median lobe of aedeagus (Fig. 9G) in ventral view fusiform, laterally clearly tapered from base to apex; ventral sulcus more clearly impressed in distal half. Spermatheca (Fig. 11M) with clearly globose basal part

A. rostrata Gómez-Zurita, 2010

- Median lobe of aedeagus (Fig. 9B) in ventral view thickset, laterally subparallel but distinctly narrowed at apical fourth; ventral sulcus obliterate. Spermatheca (Fig. 11J) with more elongate basal part, olive-shaped.

A. longifrons sp. nov.

13. Pronotum strongly transverse (WP/LP $\geq 1.83$ ) (Fig. 5D). Dorsal integuments reddish, sometimes with darker head and pronotum (Fig. 2I). Median lobe of aedeagus with triangular, apically rounded distal part (Fig. 10A). Spermatheca as in Fig. 11P.

A. transversa sp. nov.

- Pronotum less transverse (WP/LP < 1.83). Dorsal integuments bronze, dark green or blackish, with metallic lustre, or yellowish, at least on elytral disc, with weak metallic reflection (Figs 1C, G-H, 2B-D, H, 3B). Median lobe of aedeagus distally subrounded with or without median tooth, or triangular but with acute apex (Figs 8D, H-I, 9C-E, I, 10C)

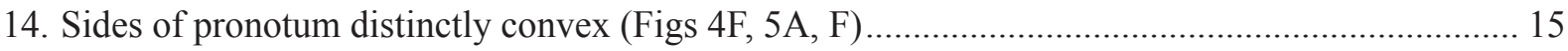

- Sides of pronotum straight or slightly concave (Fig. 4B, E) ........................................................ 17

15. Dorsal integuments blackish with metallic lustre (Fig. 2C). Median lobe of aedeagus in lateral view evenly curved from base to apex (Fig. 9D). Spermatheca as in Fig. 11K

A. paniensis sp. nov.

- Dorsal integuments yellowish (Figs 2D, 3B). Median lobe of aedeagus in lateral view sinuate or straight (Figs 9E, 10C).

16. Sides of pronotum clearly convex, with smaller and blunt anterior angles (Fig. 5F). Median lobe of aedeagus slender, laterally straight above the basal part (Fig. 10C). Collum of spermatheca thickset (Fig. 11R).

A. yiambiae Samuelson, 1973

- Sides of pronotum slightly convex, with wider and pointed anterior angles (Fig. 5A). Median lobe of aedeagus thicker, laterally sinuate (Fig. 9E). Collum of spermatheca thin (Fig. 11L) ......

A. povilaensis sp. nov.

17. Dorsal integuments evenly bronze or dark bronze with strongly metallic lustre. Hind femora generally darkened in distal half (Figs 1C, 2B). Median lobe of aedeagus slender in ventral view, and with fusiform dorsal ligula (Figs 8D, 9C).

- Dorsal integuments yellowish (in A. geographica sometimes yellowish on elytral disc only) with weakly metallic lustre. Hind femora never darkened $(1 \mathrm{G}-\mathrm{H}, 2 \mathrm{H})$. Median lobe of aedeagus thicker in ventral view, and with dorsal ligula basally wide (Figs 8H-I, 9I)

18. Body smaller (LB: $\widehat{O} \leq 2.56 \mathrm{~mm}$ and $q \leq 2.75 \mathrm{~mm}$ ). Pronotal transverse sulcus thin (Fig. 4B). Median lobe of aedeagus in ventral view laterally parallel; in lateral view, apical part strongly curved dorsally (Fig. 8D). Spermatheca as in Fig. 11C.

A. communis sp. nov.

- Body larger (LB $\delta^{\lambda}>2.56 \mathrm{~mm}$ ). Pronotal transverse sulcus large (Fig. 4E). Median lobe of aedeagus in ventral view laterally sinuate; in lateral view, apical part straight (Fig. 9C). Female unknown

A. montana sp. nov. 
19. Elytral interstriae distinctly convex (Fig. 6B). Pronotal antebasal sulcus deeply impressed (Fig. 6B). Elytral suture and lateral margin often darkened (Fig. 1G). Median lobe of aedeagus (Fig. 8H) in ventral view apically widely rounded with a very thin median small tooth; in lateral view median lobe with ventrally bent apical part. Spermatheca (Fig. 11G) with globose basal part and thickset collum

A. geographica Gómez-Zurita, 2010

- Elytral interstriae subconvex or flat (Fig. 6C). Pronotal antebasal sulcus weakly or moderately impressed (Fig. 6C). Elytra evenly yellowish (Figs 1H, 2H). Median lobe of aedeagus (Figs 8I, 9I) in ventral view apically subtriangular or rounded but with larger median tooth. Spermatheca (Fig. 11H, O) with pear-shaped or olive-shaped basal part and slender collum 20

20. Pronotum comparatively shorter (generally WP/LP: $\delta$ and $q \leq 1.55$ ). Median lobe of aedeagus (Fig. 9I) in ventral view distinctly thickset, laterally sub-parallel, clearly enlarged at apical fourth; ventral sulcus V-shaped, clearly impressed. Spermatheca (Fig. 11O) with pear-shaped basal part; collum about as long as basal part

A. shirleyae Samuelson, 1973

- Pronotum comparatively longer (generally WP/LP: $\delta$ and $q>1.55$ ). Median lobe of aedeagus (Fig. 8I) in ventral view more slender, fusiform, but narrower in pre-apical part; ventral sulcus wide and moderately impressed in basal half. Spermatheca (Fig. 11H) with olive-shaped basal part; collum clearly shorter than basal part.

A. gressitti sp. nov.

\title{
Annotated list of the Arsipoda species from New Caledonia
}

\author{
Arsipoda agalma Samuelson, 1973 \\ Figs 1A, 4A, 8A-B, 11A, 14A
}

Arsipoda agalma Samuelson, 1973: 128.

Arsipoda agalma - Gómez-Zurita et al. 2010: 2560.

\section{Type material examined}

NEW CALEDONIA: holotype đ̃, Mt Panié, [-20,58860 164,77020], 500-1000 m, 11 Oct. 1967, J. \& M. Sedlacek leg. (BPBM).

\section{New material}

NEW CALEDONIA: 1 specimen, Col d'Amieu (3.0 km to gate), -21.58536 165.79319, $500 \mathrm{~m}, 16$ Nov. 2008, M. Wanat (MNHW); 2 specimens, Parc des Grandes Fougères, N of Pic Vincent, -21.5962 165.77650, 650 m, at night, 5 Nov. 2010, M. Wanat \& R. Ruta leg. (MNHW); 1 specimen, Haute Rivière Bleue, $22^{\circ} 05^{\prime}$ S $166^{\circ} 38^{\prime}$ E, 180-330 m, track to La Tranchée, 22-23 Jan. 2004, M. Wanat leg. (MNHW); 1 specimen, ditto, La Tranchée-Sentier des Kaoris, $22^{\circ} 05^{\prime} \mathrm{S} 166^{\circ} 38^{\prime}$ E, 280-330 m, humid forest, 28 Jan. 2004, M. Wanat leg. (MNHW); 1 specimen, Riviere Bleue Parc, 1 km E of scient. Refuge, $22^{\circ} 05.9^{\prime} \mathrm{S} 166^{\circ} 39.2^{\prime} \mathrm{E}, 185 \mathrm{~m}$, humid forest, 22 Dec. 2006, R. Dobosz \& M. Wanat leg. (USMB); 1 specimen, Mt Panié, E trail, -20.5886 164.7702, 1350-1629 m, rainforest, 24 Nov. 2010, M. Wanat \& R. Ruta leg. (MNHW).

\section{Taxonomic notes}

Arsipoda agalma is quite different from the other species, mainly for the deep and elongate frontal grooves, generally continued to post-antennal region, and the weakly convex pronotum, with very large and strongly impressed punctures on disc (Fig. 4A). Median lobe of aedeagus variable (Figs 8A-B): in ventral view laterally generally parallel, or slightly narrower in apical third, with a thin median groove; apex from triangular to strongly pointed; in lateral view median lobe from thin to rather thickset, moderately curved; dorsal ligula elongate - from basal fourth to almost the apex of median lobe of aedeagus - wide, but thinner in apical part. Spermatheca (Fig. 11A) with rounded basal part; collum elongate, about twice the apical part; ductus thin, with straight and elongate proximal part. 


\title{
Biometry
}

Male ( $\mathrm{n}=6$; mean and standard deviation; range): $\mathrm{LE}=2.17 \pm 0.22 \mathrm{~mm}(1.95 \leq \mathrm{LE} \leq 2.55 \mathrm{~mm})$; $\mathrm{WE}=$ $1.41 \pm 0.14 \mathrm{~mm}(1.28 \leq \mathrm{WE} \leq 1.65 \mathrm{~mm}) ; \mathrm{LP}=0.60 \pm 0.05 \mathrm{~mm}(0.53 \leq \mathrm{LP} \leq 0.68 \mathrm{~mm}) ; \mathrm{WP}=1.06 \pm$ $0.10 \mathrm{~mm}(0.98 \leq \mathrm{WP} \leq 1.24 \mathrm{~mm}) ; \mathrm{LAN}=2.08 \pm 0.18 \mathrm{~mm}(1.80 \leq \mathrm{LAN} \leq 2.28 \mathrm{~mm}) ; \mathrm{LAED}=1.16 \pm$ $0.08 \mathrm{~mm}(1.07 \leq \mathrm{LAED} \leq 1.27 \mathrm{~mm}) ; \mathrm{LB}=3.02 \pm 0.29 \mathrm{~mm}(2.75 \leq \mathrm{LB} \leq 3.50 \mathrm{~mm}) ; \mathrm{LE} / \mathrm{LP}=3.60 \pm 0.16$ $(3.39 \leq \mathrm{LE} / \mathrm{LP} \leq 3.78) ; \mathrm{WE} / \mathrm{WP}=1.32 \pm 0.01(1.31 \leq \mathrm{WE} / \mathrm{WP} \leq 1.34) ; \mathrm{WP} / \mathrm{LP}=1.77 \pm 0.08(1.67 \leq \mathrm{WP} /$ $\mathrm{LP} \leq 1.86) ; \mathrm{WE} / \mathrm{LE}=0.65 \pm 0.01(0.63 \leq \mathrm{WE} / \mathrm{LE} \leq 0.66) ; \mathrm{LAN} / \mathrm{LB}=0.68 \pm 0.06(0.61 \leq \mathrm{LAN} / \mathrm{LB} \leq$ $0.75) ; \mathrm{LE} / \mathrm{LAED}=1.89 \pm 0.09(1.79 \leq \mathrm{LE} / \mathrm{LAED} \leq 2.01)$.

Female ( $\mathrm{n}=5$; mean and standard deviation; range): $\mathrm{LE}=2.34 \pm 0.19 \mathrm{~mm}(2.18 \leq \mathrm{LE} \leq 2.65 \mathrm{~mm})$; WE $=$ $1.57 \pm 0.16 \mathrm{~mm}(1.45 \leq \mathrm{WE} \leq 1.83 \mathrm{~mm}) ; \mathrm{LP}=0.63 \pm 0.04 \mathrm{~mm}(0.60 \leq \mathrm{LP} \leq 0.70 \mathrm{~mm}) ; \mathrm{WP}=1.15 \pm$ $0.11 \mathrm{~mm}(1.05 \leq \mathrm{WP} \leq 1.33 \mathrm{~mm}) ; \mathrm{LAN}=1.83 \pm 0.28 \mathrm{~mm}(1.53 \leq \mathrm{LAN} \leq 2.23 \mathrm{~mm}) ; \mathrm{LSPc}=0.21 \pm$ $0.02 \mathrm{~mm}(0.20 \leq \mathrm{LSPc} \leq 0.23 \mathrm{~mm}) ; \mathrm{LB}=3.16 \pm 0.30 \mathrm{~mm}(2.88 \leq \mathrm{LB} \leq 3.59 \mathrm{~mm}) ; \mathrm{LE} / \mathrm{LP}=3.72 \pm 0.09$ $(3.62 \leq \mathrm{LE} / \mathrm{LP} \leq 3.80) ; \mathrm{WE} / \mathrm{WP}=1.36 \pm 0.02(1.33 \leq \mathrm{WE} / \mathrm{WP} \leq 1.38) ; \mathrm{WP} / \mathrm{LP}=1.82 \pm 0.09(1.72 \leq \mathrm{WP} /$ $\mathrm{LP} \leq 1.92) ; \mathrm{WE} / \mathrm{LE}=0.67 \pm 0.02(0.64 \leq \mathrm{WE} / \mathrm{LE} \leq 0.69) ; \mathrm{LAN} / \mathrm{LB}=0.58 \pm 0.04(0.52 \leq \mathrm{LAN} / \mathrm{LB} \leq$ $0.62) ; \mathrm{LE} / \mathrm{LSPc}=10.93 \pm 0.61(10.24 \leq \mathrm{LE} / \mathrm{LSPc} \leq 11.62)$.

\section{Distribution}

Northern, Central and Southern Grande Terre (Fig. 14A).

\section{Ecological notes}

Collected in humid forest and rainforest, from $180 \mathrm{~m}$ to over 1350 a.s.1. No information is available about host plants. Pollen found in the gut of some specimens.

\author{
Arsipoda atra sp. nov. \\ urn:Isid:zoobank.org:act:7BDC873B-2A3E-453B-8DF8-CD3CB0806018
}

Figs 1B, 6A, 8C, 11B, 14B

\section{Diagnosis}

Arsipoda atra sp. nov. shares a thickset and ovoid body with A. evax, A. povilaensis sp. nov. and $A$. transversa sp. nov. (Figs 1B, F, 2D, I). However, the new species can easily be distinguished from other species by its black, non-metallic integuments, and wide and flat elytral interstriae. Genitalia are also highly diagnostic (Figs 8C, G, 9E, 10A, 11B, F, L, P).

\section{Etymology}

The specific epithet is a Latin adjective that means black, dark.

\section{Type material}

\section{Holotype}

NEW CALEDONIA (N): §̂, Aoupinié, near Goipin rd jct [= road junction], -21.1739 165.3116, 700 800 m, 20 Nov. 2010, M. Wanat \& R. Ruta leg. (MNHN).

\section{Paratypes}

NEW CALEDONIA: 1 , same data as the holotype (MNHW); 1 q, Aoupinié, -21.18151 165.30048, 790-830 m, 27 Nov. 2010, M. Wanat leg. (MNHW).

\section{Description of the holotype $(ふ)$}

Body thickset, moderately convex (Fig. 1B); LB $=2.25 \mathrm{~mm}$; maximum pronotal width at base $(\mathrm{WP}=0.90 \mathrm{~mm})$; maximum elytral width at basal third $(\mathrm{WE}=1.18 \mathrm{~mm})$. Dorsum blackish, non- 

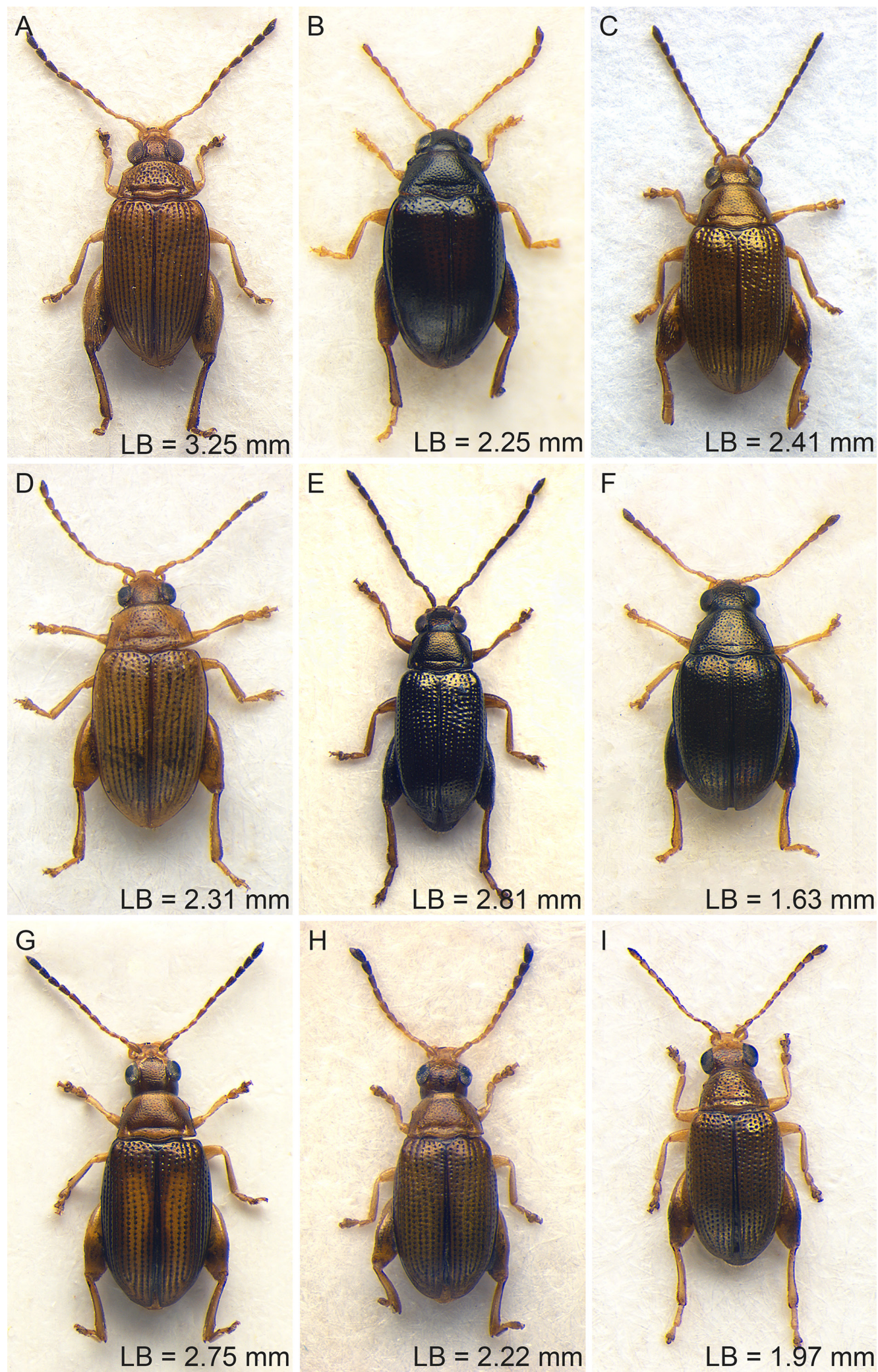

Fig. 1. Habitus. A. Arsipoda agalma Samuelson, 1973, Mt Panié. B. A. atra sp. nov, Aoupinié. C. A. communis sp. nov, Mt Do. D. A. doboszi sp. nov, Haute Rivière Bleue. E. A. elongata sp. nov, Mt Humboldt. F. A. evax Samuelson, 1973, Koghi Mts. G. A. geographica Gómez-Zurita, 2010, Haute Rivière Bleue. H. A. gressitti sp. nov., Farino. I. A. isola Samuelson, 1973, Bois du Sud. 
metallic, paler on elytral disc and anterior part of pronotum. Frons and vertex finely microreticulate and finely punctate; head grooves moderately impressed, continued to postantennal region; genae and frontal carina short; antennae slightlty longer than $1 / 2$ body length (LAN $=1.35 \mathrm{~mm}$; LAN/ $\mathrm{LB}=0.60$ ), yellowish, gradually darker towards apex; LA: 100:77:64:64:91:82:91:91:91:91:132. Pronotum trapezoidal, distinctly transverse $(\mathrm{LP}=0.50 \mathrm{~mm}$; WP $/ \mathrm{LP}=1.80)$, laterally straight; anterior angles distinctly prominent; antebasal transverse sulcus weakly impressed, weakly sinuate, without clearly distinguishable lateral fovea; pronotal surface microreticulate, opaque, with small, dense punctures, distinctly impressed. Elytra slightly elongate $(\mathrm{LE}=1.73 \mathrm{~mm} ; \mathrm{WE} / \mathrm{LE}=0.68)$, laterally clearly arcuate (Fig. 6A); punctuation slightly larger than on pronotum; surface finely microreticulate; interstriae regularly punctulate. Anterior and middle legs yellowish; hind legs brown; first pro- and mesotarsomeres moderately dilated; first metatarsomere in male distally slightly expanded on inner side; adhesive setae present on ventral side of first pro-, meso- and, partially, metatarsomeres. Venter brown; last abdominal ventrite laterally clearly incised, without special preapical impressions. Median lobe of aedeagus $(\mathrm{LAED}=1.03 \mathrm{~mm}$; LE/LAED $=1.67)($ Fig. $8 \mathrm{C})$ in ventral view laterally parallel, slightly narrower at basal $2 / 5 \mathrm{~S}$ and toward apex; apex flat, elongate, with rounded margin; ventral surface with shallow median groove and two thin lateral carinae; in lateral view, median lobe strongly bent at basal fourth, with sinuate apical part; dorsal ligula quite short - from apical $2 / 5$ s to pre-apical part of aedeagus - , thin, tapered towards apex.

\section{Biometry}

Male $(\mathrm{n}=1): \mathrm{LE}=1.73 \mathrm{~mm} ; \mathrm{WE}=1.18 \mathrm{~mm} ; \mathrm{LP}=0.50 \mathrm{~mm} ; \mathrm{WP}=0.90 \mathrm{~mm} ; \mathrm{LAN}=1.35 \mathrm{~mm} ; \mathrm{LAED}=$ $1.03 \mathrm{~mm} ; \mathrm{LB}=2.25 \mathrm{~mm} ; \mathrm{LE} / \mathrm{LP}=3.45 ; \mathrm{WE} / \mathrm{WP}=1.31 ; \mathrm{WP} / \mathrm{LP}=1.80 ; \mathrm{WE} / \mathrm{LE}=0.68 ; \mathrm{LAN} / \mathrm{LB}=$ $0.60 ; \mathrm{LE} / \mathrm{LAED}=1.67$.

Female $(\mathrm{n}=2): \mathrm{LE}=1.95,1.73 \mathrm{~mm} ; \mathrm{WE}=1.33,1.20 \mathrm{~mm} ; \mathrm{LP}=0.58,0.50 \mathrm{~mm}$; $\mathrm{WP}=1.01,0.89 \mathrm{~mm}$; $\mathrm{LAN}=1.35,1.25 \mathrm{~mm} ; \mathrm{LSPc}=0.16,0.16 \mathrm{~mm} ; \mathrm{LB}=2.59,2.25 \mathrm{~mm} ; \mathrm{LE} / \mathrm{LP}=3.39,3.45 ; \mathrm{WE} / \mathrm{WP}=$ $1.31,1.35 ; \mathrm{WP} / \mathrm{LP}=1.76,1.78 ; \mathrm{WE} / \mathrm{LE}=0.68,0.70 ; \mathrm{LAN} / \mathrm{LB}=0.52,0.56 ; \mathrm{LE} / \mathrm{LSPc}=12.50,10.78$.

Paratypes (females) very similar in shape and sculpture to the holotype. Dorsum evenly black. Antennae slightly shorter. Spermatheca (Fig. 11B) with small, subrounded basal part; collum thickened, tapered towards apical part, elongate; ductus thin, elongate, preapically inserted on dorsal side, single-coiled.

\section{Distribution}

Endemic to the Aoupinié area, Central Grande Terre (Fig. 14B).

\section{Ecological notes}

No ecological notes are available for this species, but collecting localities are located in a dense, evergreen humid forest area between 700 and $830 \mathrm{~m}$ a.s.1. Pollen found in the gut of some specimens.

$$
\begin{aligned}
& \text { Arsipoda communis sp. nov. } \\
& \text { urn:1sid:zoobank.org:act:9163B82F-1FBB-4E76-9609-071C64294EEF }
\end{aligned}
$$

Figs 1C, 4B, 6E(1), F, 8D, 11C, T, 14B

\section{Diagnosis}

Arsipoda communis sp. nov. is a bronze, strongly metallic species quite similar in sculpture and color to $A$. montana sp. nov. It can be distinguishable by the less transverse pronotum and the more weakly impressed pronotal transverse sulcus (Fig. 4B, E). Median lobe of aedeagus is also useful to discriminate between the two species (Figs 8D, 9C). 
D’ALESSANDRO P. et al., Arsipoda Erichson, 1842 from New Caledonia

\section{Etymology}

The specific epithet is a Latin adjective referring to its relatively wide distribution in New Caledonia.

\section{Type material}

\section{Holotype}

NEW CALEDONIA: Oૈ, Mt Do, -21.75706 165.99894, 920 m, at light, 6 Nov. 2008, M. Wanat leg. (MNHN).

\section{Paratypes}

NEW CALEDONIA: 2 ๙ึ, 6 우, Mt Do, -21.75585 166.00099, 900-1025 m, maquis and forest edge, 6 Nov. 2008, M. Wanat leg. (MNHW); 17 $\widehat{\jmath}, 17$ 우, ditto, 900-1000 m, roadside, night beating (MNHW); 1 \&, ditto, -21.75440 165.99950, 1025 m, summit maquis, 4 Nov. 2010, M. Wanat and R.

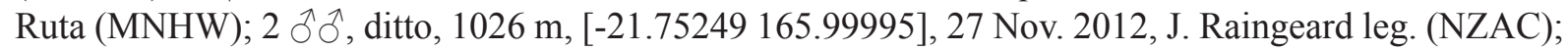
2 ふึ, 5 우우, ditto, [-21.75706 165.99894], $1000 \mathrm{~m}$, beating at night, 31 Oct. 1978, J.C. Watt leg. (BPBM, NZAC); 1 9, ditto, -21.75740 166.00150, 850-950 m, day beating, 3 Nov. 2010 (MNHW);

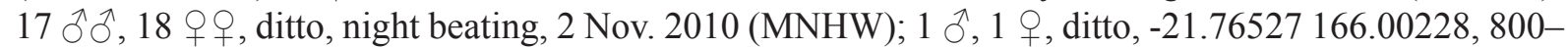

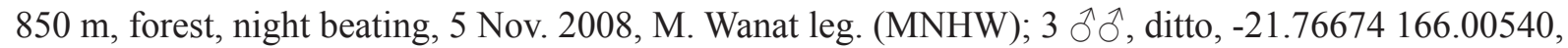

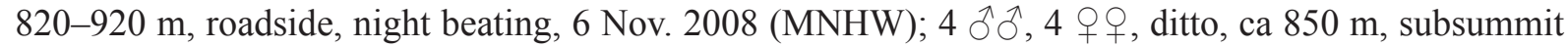
forest, night, 5 Nov. 2008 (MNHW); 2 우, ditto, -21,76060, 165,99960, 850 m, subsummit forest, at

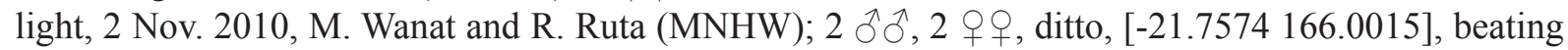
Araucaria laubenfelsii Corbasson branches, 21 Nov. 2002, R. Mecke leg., coll. Christian Mille (BAQ); 1 ô, 3 우, Boulouparis/Mont Do, -21.75394 161.99994 [165.99994], 1029 m, battage, collecté sur Araucaria laubenfelsii, 6 Oct. 2004, S. Cazères and C. Mille leg., coll. Christian Mille (BAQ); 1 q,

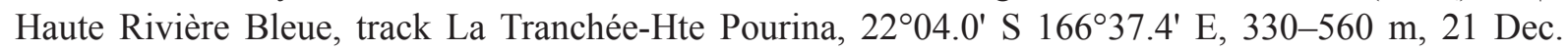
2006, M. Wanat leg. (MNHW); 1 क, Tontouta River, -21.94861 166.28834, $30 \mathrm{~m}$, at light, 1 Nov. 2008, M. Wanat leg. (MNHW); 3 우, Plaine du Champ de Bataille, $22^{\circ} 18.1^{\prime} \mathrm{S} 166^{\circ} 44.7^{\prime} \mathrm{E}, 40 \mathrm{~m}$, maquis, 17 Dec. 2006, M. Wanat, R. Dobosz and P. Krzyzynski leg. (MNHW); 1 §, 1 , , Dzumac road,

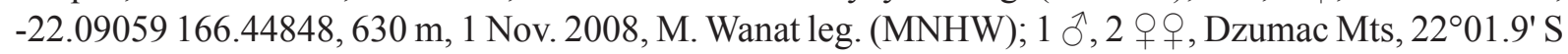
$166^{\circ} 28.0^{\prime} \mathrm{E}, 900 \mathrm{~m}$, netting, 28 Dec. 2006, R. Dobosz leg. (USMB); $1 \mathrm{o}^{\Uparrow}$, ditto, road from jet to old mine

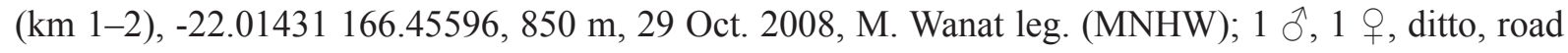
from jet to old mine ( $\mathrm{km} \mathrm{0-1),} 22.02051$ 166.46606, 850-910 m, 29 Oct. 2008 (MNHW); 1 , ditto, Mt Ouin road junction, -22.03188 166.46738, 900 m, 31 Oct. 2008, M. Wanat leg. (MNHW); 1 ô, 1 \%,

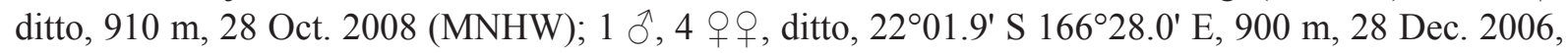

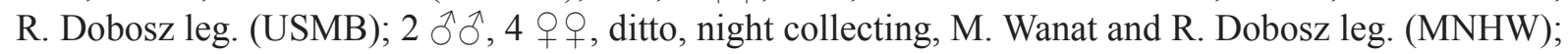

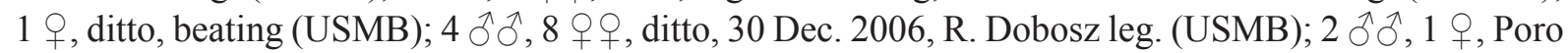
Plateau, -21.34832 165.69322, 620 m, forest, night, 27 Nov. 2010, M. Wanat and R. Ruta leg. (MNHW);

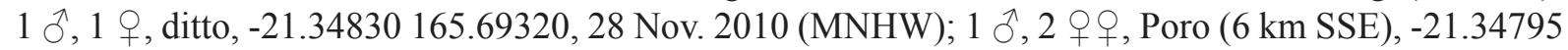
165.69385, $620 \mathrm{~m}$, plateau above mine, forest, 30 Nov. 2008, M. Wanat leg. (MNHW); 1 , Mt Koghi, $22^{\circ} 10.7^{\prime}$ S $166^{\circ} 30.4^{\prime}$ E, 450-500 m, rainforest, netting, 16 Dec. 2006, R. Dobosz leg. (USMB); 1 ð, ditto, [-22.17236 166.51129], 400-600 m, Jan.1969, N.L.H. Krauss leg. (BPBM); 1 , Mountain stream up Boulari River [-22.23333 166,56667], light trap, 3 Nov. 1958, C.R. Joyce leg. (BPBM); 1 $\Uparrow, 3$ 우우, Valle de Thy, [-22.18330 166.53300], 250-500 m, 28-29 Mar. 1981, J.L. Gressitt and G.M. McPherson leg. (BPBM); 1 옹 Plateau de Dogny, [-21.60987 165.88907], 1000 m, on Metrosideros operculata Labill., 11 Apr. 1973, J.L. Gressitt leg. (BPBM); 13 đ̊̊, 10 우, Pau Bâ Moneo, [-21.18386 165.51701],

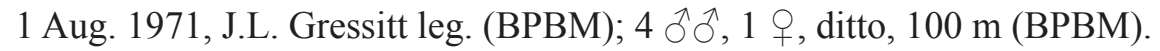

\section{Description of the holotype $(\precsim)$}

Body moderately elongate and convex (Fig. 1C); $\mathrm{LB}=2.25 \mathrm{~mm}$; maximum pronotal width at base (WP = $0.78 \mathrm{~mm})$; maximum elytral width at basal third $(\mathrm{WE}=1.06 \mathrm{~mm})$. Dorsum bronze, metallic. Frons 
and vertex microreticulate and densely punctate; frontal grooves very weakly impressed, not continued to postantennal region; genae and frontal carina short; antennae slightly longer than $1 / 2$ body length $(\mathrm{LAN}=1.44 \mathrm{~mm} ; \mathrm{LAN} / \mathrm{LB}=0.64)$, brownish, slightly paler basally; LA: 100:67:58:58:83:83:92:92:9 2:92:125. Pronotum trapezoidal, distinctly transverse $(\mathrm{LP}=0.48 \mathrm{~mm} ; \mathrm{WP} / \mathrm{LP}=1.63)$, laterally straight (Fig. 4B); anterior angles slightly prominent; antebasal transverse sulcus thin but clearly impressed, straight; lateral fovea distinctly impressed; pronotal punctuation small and shallow, similar to head punctures, dense, evenly distributed on the densely microreticulate surface. Elytra moderately elongate $(\mathrm{LE}=1.67 \mathrm{~mm} ; \mathrm{WE} / \mathrm{LE}=0.63)$, laterally moderately arcuate; punctuation strongly impressed, clearly larger than on pronotum; interstriae microreticulate and micropunctuate. Legs yellowish; hind femora distally darkened; first pro- and mesotarsomeres distinctly dilated; adhesive setae present on ventral side of first pro-, meso- and, partially, metatarsomeres. Venter dark brown; last abdominal ventrite laterally clearly incised, without special preapical impressions. Median lobe of aedeagus (LAED $=1.10 \mathrm{~mm}$; LE/LAED = 1.52) (Fig. 8D) in ventral view laterally parallel, apically rounded, with a wide, rounded median tooth; ventral surface with a deep median groove with sinuate lateral margins; in lateral view median lobe strongly sinuate, with dorsally bent apical part; dorsal ligula fusiform, narrow, from half length to apical part of median lobe of aedeagus.

\section{Biometry}

Male ( $\mathrm{n}=10$; mean and standard deviation; range): $\mathrm{LE}=1.72 \pm 0.09 \mathrm{~mm}(1.58 \leq \mathrm{LE} \leq 1.88 \mathrm{~mm})$; $\mathrm{WE}=$ $1.11 \pm 0.06 \mathrm{~mm}(1.03 \leq \mathrm{WE} \leq 1.18 \mathrm{~mm}) ; \mathrm{LP}=0.49 \pm 0.02 \mathrm{~mm}(0.45 \leq \mathrm{LP} \leq 0.51 \mathrm{~mm}) ; \mathrm{WP}=0.80 \pm$ $0.04 \mathrm{~mm}(0.73 \leq \mathrm{WP} \leq 0.85 \mathrm{~mm}) ; \mathrm{LAN}=1.49 \pm 0.07 \mathrm{~mm}(1.38 \leq \mathrm{LAN} \leq 1.58 \mathrm{~mm}) ; \mathrm{LAED}=1.09 \pm$ $0.04 \mathrm{~mm}(1.03 \leq \mathrm{LAED} \leq 1.13 \mathrm{~mm}) ; \mathrm{LB}=2.38 \pm 0.14 \mathrm{~mm}(2.19 \leq \mathrm{LB} \leq 2.56 \mathrm{~mm}) ; \mathrm{LE} / \mathrm{LP}=3.52 \pm 0.08$ $(3.44 \leq \mathrm{LE} / \mathrm{LP} \leq 3.70) ; \mathrm{WE} / \mathrm{WP}=1.39 \pm 0.03(1.34 \leq \mathrm{WE} / \mathrm{WP} \leq 1.43) ; \mathrm{WP} / \mathrm{LP}=1.63 \pm 0.04(1.58 \leq \mathrm{WP} /$ $\mathrm{LP} \leq 1.70) ; \mathrm{WE} / \mathrm{LE}=0.64 \pm 0.01(0.62 \leq \mathrm{WE} / \mathrm{LE} \leq 0.67) ; \mathrm{LAN} / \mathrm{LB}=0.63 \pm 0.02(0.59 \leq \mathrm{LAN} / \mathrm{LB} \leq$ $0.66) ; \mathrm{LE} / \mathrm{LAED}=1.58 \pm 0.05(1.52 \leq \mathrm{LE} / \mathrm{LAED} \leq 1.69)$.

Female ( $\mathrm{n}=10$; mean and standard deviation; range): $\mathrm{LE}=1.86 \pm 0.14 \mathrm{~mm}(1.63 \leq \mathrm{LE} \leq 2.04 \mathrm{~mm})$; $\mathrm{WE}=1.22 \pm 0.11 \mathrm{~mm}(1.03 \leq \mathrm{WE} \leq 1.35 \mathrm{~mm}) ; \mathrm{LP}=0.51 \pm 0.05 \mathrm{~mm}(0.43 \leq \mathrm{LP} \leq 0.58 \mathrm{~mm}) ; \mathrm{WP}=$ $0.86 \pm 0.07 \mathrm{~mm}(0.73 \leq \mathrm{WP} \leq 0.95 \mathrm{~mm}) ; \mathrm{LAN}=1.39 \pm 0.11 \mathrm{~mm}(1.15 \leq \mathrm{LAN} \leq 1.50 \mathrm{~mm}) ; \mathrm{LSPc}=$ $0.20 \pm 0.01 \mathrm{~mm}(0.18 \leq \mathrm{LSPc} \leq 0.21 \mathrm{~mm}) ; \mathrm{LB}=2.52 \pm 0.19 \mathrm{~mm}(2.13 \leq \mathrm{LB} \leq 2.75 \mathrm{~mm}) ; \mathrm{LE} / \mathrm{LP}=$ $3.62 \pm 0.11(3.49 \leq \mathrm{LE} / \mathrm{LP} \leq 3.82) ; \mathrm{WE} / \mathrm{WP}=1.42 \pm 0.04(1.34 \leq \mathrm{WE} / \mathrm{WP} \leq 1.46) ; \mathrm{WP} / \mathrm{LP}=1.67 \pm 0.04$ $(1.60 \leq \mathrm{WP} / \mathrm{LP} \leq 1.75) ; \mathrm{WE} / \mathrm{LE}=0.65 \pm 0.01(0.63 \leq \mathrm{WE} / \mathrm{LE} \leq 0.67) ; \mathrm{LAN} / \mathrm{LB}=0.55 \pm 0.01(0.53 \leq$ $\mathrm{LAN} / \mathrm{LB} \leq 0.58) ; \mathrm{LE} / \mathrm{LSPc}=9.41 \pm 0.47(8.83 \leq \mathrm{LE} / \mathrm{LSPc} \leq 10.42)$.

Paratypes very similar in shape and sculpture to the holotype; some specimens slightly paler or darker. Antennae slightly shorter in female (LAN/LB in male $=0.63 \pm 0.02$; in female $=0.55 \pm 0.01$ ). Spermatheca (Fig. 11C) with large, ovate basal part; collum moderately elongate; apical part shorter than collum; ductus moderately elongate, straight in proximal part.

\section{Distribution}

Central and Southern Grande Terre (Fig. 14B).

\section{Ecological notes}

Collected in forest, forest edges and maquis between 100 and $1025 \mathrm{~m}$ a.s.1. Found on Araucaria laubenfelsii (Auracariaceae), Neoguillauminia sp. (Euphorbiaceae), Metrosideros operculata (Myrtaceae). Pollen found in the gut of some specimens. 


\section{Arsipoda doboszi sp. nov. urn:1sid:zoobank.org:act:5DC99F7F-5C6E-4AC1-B474-86275A16601C}

Figs 1D, 4C, 8E, 11D, 15B

\section{Diagnosis}

Yellow species, easily distinguishable by the sparsely and unevenly distributed pronotal punctuation (Fig. 4C), and the smaller size compared to the other yellowish species.

\section{Etymology}

Male patronym. Named after Dr. Roland Dobosz (Upper Silesian Museum, Bytom, Poland), one of its collectors.

\section{Type material}

\section{Holotype}

NEW CALEDONIA(S): ${ }^{\lambda}$, Mt Dzumac Road ( $\left.7^{\text {th }} \mathrm{km}\right), 22^{\circ} 05^{\prime} \mathrm{S} 166^{\circ} 27^{\prime} \mathrm{E}, 700 \mathrm{~m}$, maquis, ex Hibbertia lucens Brongn. \& Gris, 9 Feb. 2004, M. Wanat leg. (MNHN).

\section{Paratypes}

NEW CALEDONIA: 1 § , Dzumac Mts, -22.02051 166.46606, 850-910 m, road from jct to old mine (km

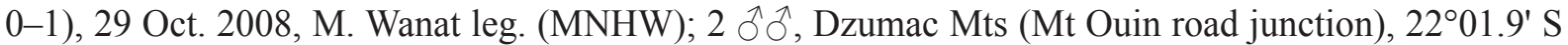

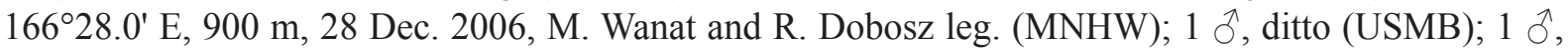

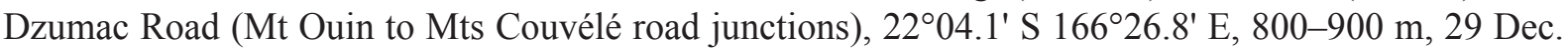
2006, M. Wanat leg. (MNHW); 1 ô, Dzumac Road, S of Mts Couvélé rd jct [= road junction], -2.06913 166.44666, 870-670 m, beating, 31 Oct. 2008, M. Wanat leg. (MNHW); 1 +, Dzumac road, -22.08783 166.44643, 650-700 m, 1 Nov. 2008, M. Wanat leg. (MNHW); 1 ô, Mt Ouin Rd, km 0-0.5 N of Dzumac jct, -22.03180 166.46740, 900 m, 4 Dec. 2010, R. Ruta and M. Wanat leg. (MNHW); 1 , Mt Do, -21.76527 166.00228, 800-850 m, forest, night beating, 5 Nov. 2008, M. Wanat leg. (MNHW); 1 गे, 1 ․ Mt Do, -21.7574 166.0015, 850-950 m, night beating, 2 Nov. 2010, M. Wanat and R. Ruta leg. (MNHW); 2 ふぇ, Mt Do, -21.76060 165.99960, $850 \mathrm{~m}$, subsummit forest, at light, 2 Nov. 2010, M. Wanat and R. Ruta leg. (MNHW); 2 $\widehat{\partial}, 1$, , Haute Rivière Bleue, track La Tranchée- $\mathrm{H}^{\text {te }}$ Pourina, $22^{\circ} 04.0^{\prime} \mathrm{S} 166^{\circ} 37.4^{\prime} \mathrm{E}, 330-560$ m, 21 Dec. 2006, M. Wanat leg. (MNHW); 1 ㅇ, Haute Rivière Bleue, La Tranchée - Sentier des Kaoris, 22 $2^{\circ} 05^{\prime}$ S $166^{\circ} 38^{\prime}$ E, 280-330 m, humid forest, 26 Jan. 2004, M. Wanat

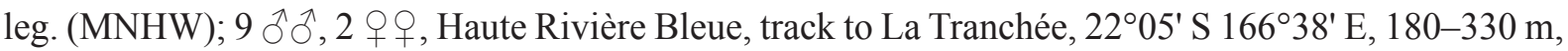
22-23 Jan. 2004, M. Wanat leg. (MNHW); 1 đ, 1 क , ditto, 190-330 m, 20 Dec. 2006, M. Wanat and R. Dobosz leg. (MNHW); 1 q , ditto, 21 Dec. 2006, R. Dobosz leg. (USMB); 1 ${ }^{2}, 1$ q, Rivière Bleue, N of La Tranchée, $22^{\circ} 05^{\prime} \mathrm{S} 166^{\circ} 38^{\prime} \mathrm{E}, 280-330 \mathrm{~m}, 20$ Dec. 2006, R. Dobosz and M. Wanat leg. (USMB); 2 우, S Humboldt, trail to Col du Vulcain, -21.90430 166.38100, 800-950 m, maquis, 11 Nov. 2010, M. Wanat and R. Ruta leg. (MNHW); 1 o, Koghi Mts, -22.17631 166.50138, 340 m, roadside, 25 Oct.

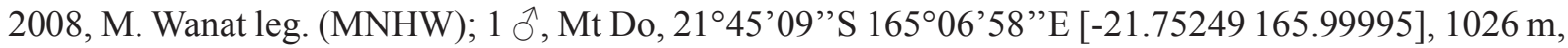
27 Nov. 2012, J. Raingeard leg. (NZAC).

\section{Description of the holotype (ð)}

Body moderately elongate, weakly convex (Fig. $1 \mathrm{D}$ ); $\mathrm{LB}=2.31 \mathrm{~mm}$; maximum pronotal width at base $(\mathrm{WP}=0.81 \mathrm{~mm})$; maximum elytral width at basal third $(\mathrm{WE}=1.08 \mathrm{~mm})$. Dorsum yellowish, not metallic, with scutellum, elytral suture and elytral punctuation slightly darkened. Frons and vertex microreticulate, with small, shallow punctures; head grooves distinctly impressed, not continued to postantennal region (Fig. 4C); genae and frontal carina short; antennae about as long as $1 / 2$ body length $(\mathrm{LAN}=1.28 \mathrm{~mm} ; \mathrm{LAN} / \mathrm{LB}=0.55)$, basally yellow, gradually and slightly darker towards apex; LA: 100:64:54:64:82:89:91:100:100:100:118. Pronotum trapezoidal, transverse $(\mathrm{LP}=0.48 \mathrm{~mm} ; \mathrm{WP} / \mathrm{LP}=$ 
1.71), laterally straight (Fig. 4C); anterior angles distinctly prominent; antebasal transverse sulcus moderately impressed, slightly sinuate, with small lateral fovea; pronotal surface microreticulate, with clear, unevenly distributed punctuation. Elytra moderately elongate $(\mathrm{LE}=1.68 \mathrm{~mm}$; WE/LE $=0.64)$, laterally slightly arcuate; punctuation slightly larger than on pronotum and clearly impressed; interstriae very finely microreticulate and sparsely micropunctuate.

Legs yellow; first pro- and mesotarsomeres distinctly dilated; adhesive setae present on ventral side of first pro-, meso- and, partially, metatarsomeres. Venter light brown; last abdominal ventrite laterally clearly incised, without special preapical impressions. Median lobe of aedeagus (LAED $=0.89 \mathrm{~mm}$; LE/ $\mathrm{LAED}=1.88)($ Fig. $8 \mathrm{E}$ ) in ventral view tapered from basal third toward apex; basal part narrower; apex subtriangular, slightly rounded, with a median rounded small tooth; ventral surface with median sulcus basally wide and two prelateral carinae; in lateral view, median lobe thin, strongly bisinuate; dorsal ligula from half length to apical part of median lobe of aedeagus, wide, tapered toward apex.

\section{Biometry}

Male ( $\mathrm{n}=10$; mean and standard deviation; range): $\mathrm{LE}=1.63 \pm 0.07 \mathrm{~mm}(1.55 \leq \mathrm{LE} \leq 1.78 \mathrm{~mm}) ; \mathrm{WE}=$ $1.06 \pm 0.05 \mathrm{~mm}(1.00 \leq \mathrm{WE} \leq 1.15 \mathrm{~mm}) ; \mathrm{LP}=0.47 \pm 0.02 \mathrm{~mm}(0.43 \leq \mathrm{LP} \leq 0.50 \mathrm{~mm}) ; \mathrm{WP}=0.79 \pm$ $0.04 \mathrm{~mm}(0.73 \leq \mathrm{WP} \leq 0.85 \mathrm{~mm}) ; \mathrm{LAN}=1.29 \pm 0.03 \mathrm{~mm}(1.23 \leq \mathrm{LAN} \leq 1.35 \mathrm{~mm}) ; \mathrm{LAED}=0.89 \pm$ $0.02 \mathrm{~mm}(0.86 \leq \mathrm{LAED} \leq 0.90 \mathrm{~mm}) ; \mathrm{LB}=2.24 \pm 0.13 \mathrm{~mm}(2.09 \leq \mathrm{LB} \leq 2.47 \mathrm{~mm}) ; \mathrm{LE} / \mathrm{LP}=3.50 \pm 0.08$ $(3.37 \leq \mathrm{LE} / \mathrm{LP} \leq 3.65) ; \mathrm{WE} / \mathrm{WP}=1.35 \pm 0.02(1.32 \leq \mathrm{WE} / \mathrm{WP} \leq 1.38) ; \mathrm{WP} / \mathrm{LP}=1.69 \pm 0.03(1.63 \leq \mathrm{WP} /$ $\mathrm{LP} \leq 1.74) ; \mathrm{WE} / \mathrm{LE}=0.65 \pm 0.01(0.63 \leq \mathrm{WE} / \mathrm{LE} \leq 0.68) ; \mathrm{LAN} / \mathrm{LB}=0.58 \pm 0.03(0.54 \leq \mathrm{LAN} / \mathrm{LB} \leq$ $0.62) ; \mathrm{LE} / \mathrm{LAED}=1.84 \pm 0.06(1.77 \leq \mathrm{LE} / \mathrm{LAED} \leq 1.98)$.

Female ( $\mathrm{n}=10$; mean and standard deviation; range): $\mathrm{LE}=1.75 \pm 0.10 \mathrm{~mm}(1.60 \leq \mathrm{LE} \leq 1.90 \mathrm{~mm})$; $\mathrm{WE}=1.11 \pm 0.07 \mathrm{~mm}(1.00 \leq \mathrm{WE} \leq 1.25 \mathrm{~mm}) ; \mathrm{LP}=0.48 \pm 0.03 \mathrm{~mm}(0.43 \leq \mathrm{LP} \leq 0.51 \mathrm{~mm}) ; \mathrm{WP}=$ $0.81 \pm 0.05 \mathrm{~mm}(0.74 \leq \mathrm{WP} \leq 0.89 \mathrm{~mm}) ; \mathrm{LAN}=1.27 \pm 0.10 \mathrm{~mm}(1.08 \leq \mathrm{LAN} \leq 1.40 \mathrm{~mm}) ; \mathrm{LSPc}=$ $0.23 \pm 0.01 \mathrm{~mm}(0.22 \leq \mathrm{LSPc} \leq 0.24 \mathrm{~mm}) ; \mathrm{LB}=2.40 \pm 0.15 \mathrm{~mm}(2.22 \leq \mathrm{LB} \leq 2.59 \mathrm{~mm}) ; \mathrm{LE} / \mathrm{LP}=$ $3.69 \pm 0.10(3.53 \leq \mathrm{LE} / \mathrm{LP} \leq 3.80) ; \mathrm{WE} / \mathrm{WP}=1.37 \pm 0.02(1.33 \leq \mathrm{WE} / \mathrm{WP} \leq 1.41) ; \mathrm{WP} / \mathrm{LP}=1.71 \pm 0.05$ $(1.63 \leq \mathrm{WP} / \mathrm{LP} \leq 1.74) ; \mathrm{WE} / \mathrm{LE}=0.63 \pm 0.02(0.62 \leq \mathrm{WE} / \mathrm{LE} \leq 0.67) ; \mathrm{LAN} / \mathrm{LB}=0.53 \pm 0.02(0.48 \leq$ $\mathrm{LAN} / \mathrm{LB} \leq 0.56) ; \mathrm{LE} / \mathrm{LSPc}=7.72 \pm 0.39(7.16 \leq \mathrm{LE} / \mathrm{LSPc} \leq 8.37)$.

Paratypes very similar in shape, sculpture and color to the holotype. Spermatheca (Fig. 11D) with elongate, slightly reniform basal part; collum wrinkled, elongate, slightly longer than apical part; ductus short, apically inserted.

\section{Distribution}

Southern Grande Terre (Fig. 15B).

\section{Ecological notes}

Collected in subsummit forest, humid forest and maquis between 180 and $950 \mathrm{~m}$ a.s.l. Found on Hibbertia lucens (Dilleniaceae). Pollen found in the gut of some specimens.

Arsipoda elongata sp. nov. urn:lsid:zoobank.org:act:97EB34B7-866E-4D3F-ACBE-5341B904489E

Figs 1E, 8F, 11E, 15A

\section{Diagnosis}

Arsipoda elongata sp. nov. shares the elongate antennae with A. gomezzuritai sp. nov., A. longifrons sp. nov. and $A$. rostrata (LAN/LB: $\widehat{\delta} \geq 0.70$, + generally $\geq 0.60$ ) (Figs 1E, 2A, F, 3C). However, it can easily be distinguished from them by the elongate, laterally parallel elytra, and by the green metallic color. 


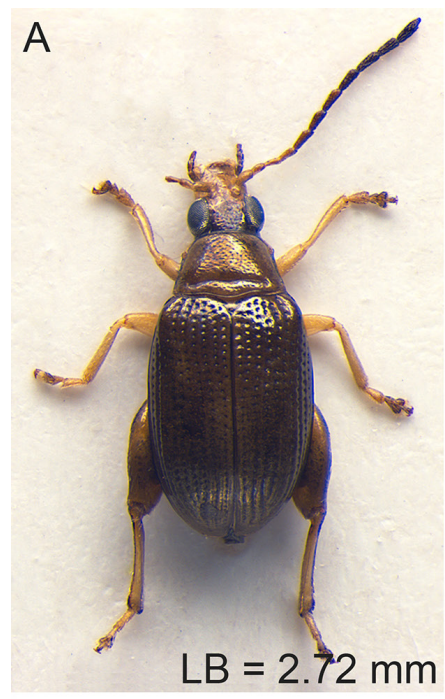

B

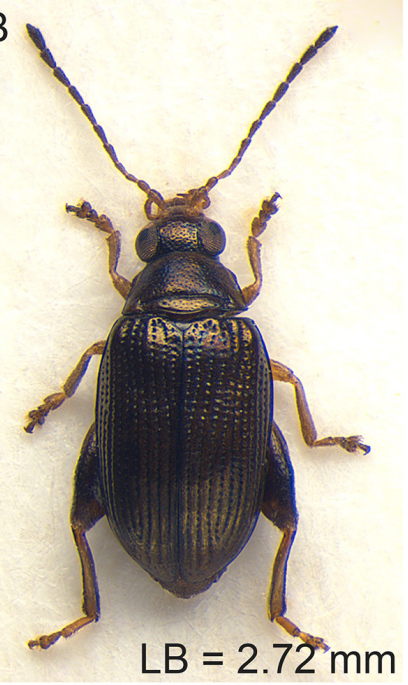

D

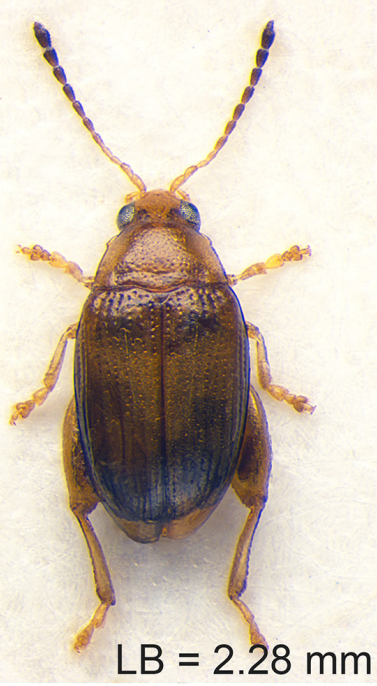

E

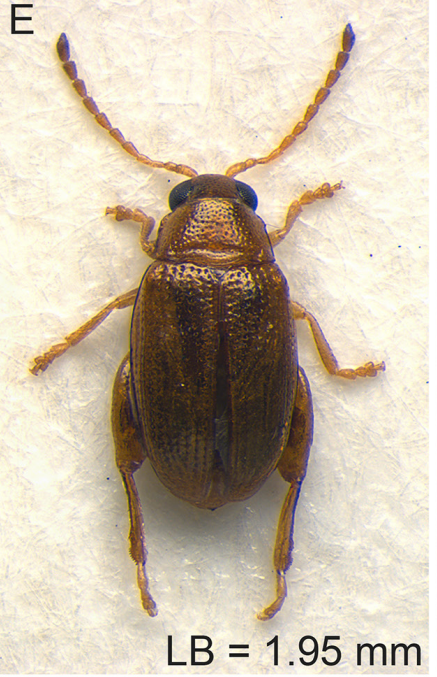

C

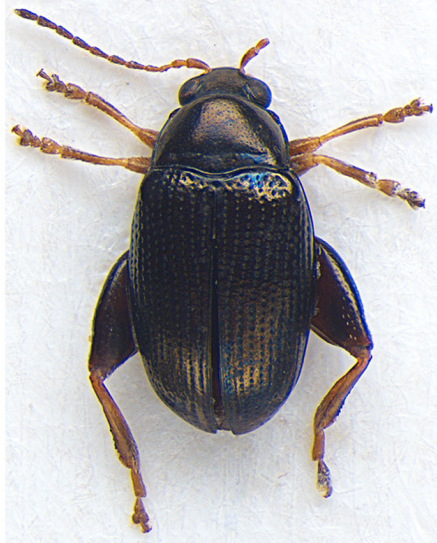

$\mathrm{LB}=2.53 \mathrm{~mm}$

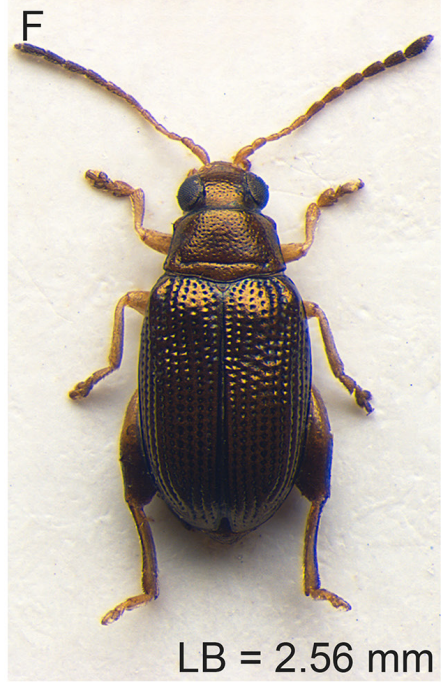

G
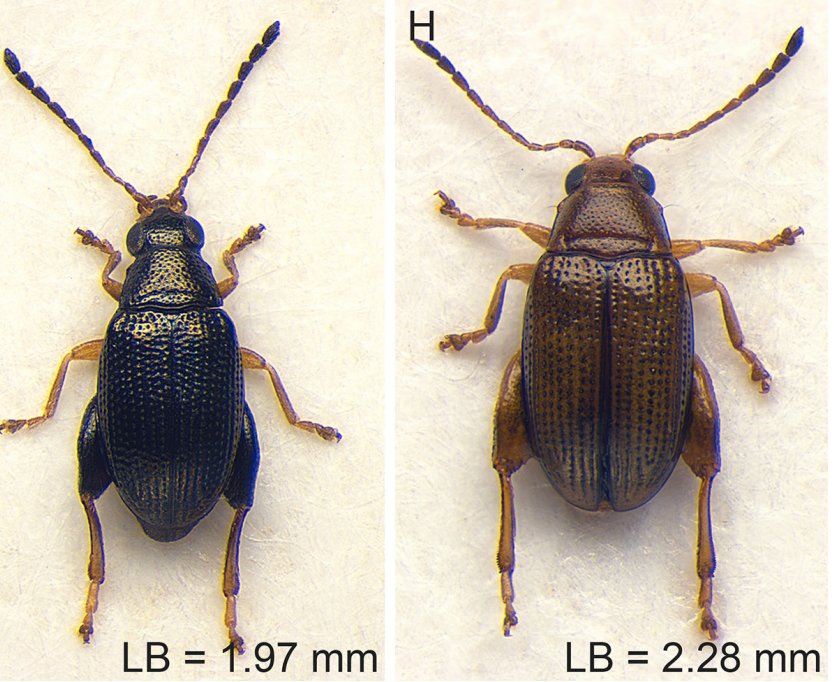

1

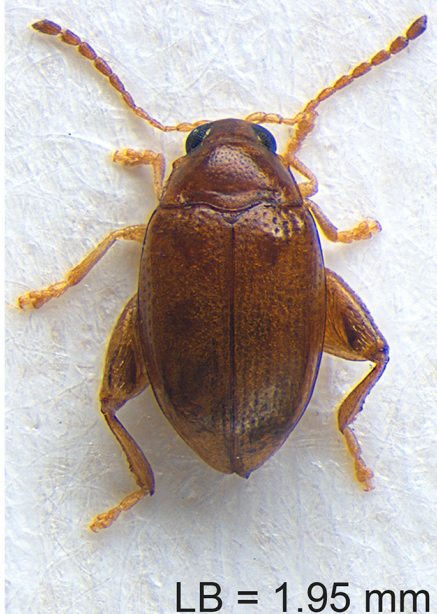

Fig. 2. Habitus. A. Arsipoda longifrons sp. nov, Humboldt S track. B. A. montana sp. nov, Mt Humboldt. C. A. paniensis sp. nov, Mt Panié. D. A. povilaensis sp. nov, Pic d'Amoa. E. A. punctata sp. nov., Mueo. F. A. rostrata Gómez-Zurita, 2010, Koghi Mts. G. A. rutai sp. nov, Poro Plateau. H. A. shirleyae Samuelson, 1973, S Humboldt. I. A. transversa sp. nov., Mt Koghi. 


\section{Etymology}

The specific name is a Latinized adjective referring to the elongate elytra of this species.

\section{Type material}

\section{Holotype}

NEW CALEDONIA (S): đ̊ Mt Humboldt, -21.8793 166.4232, ca 1400 m, montane maquis, 13 Nov. 2010, R. Ruta \& M. Wanat leg. (MNHN).

\section{Paratypes}

NEW CALEDONIA: $2 \widehat{\jmath}, 5$ $q+$, same data as the holotype (MNHW).

\section{Description of the holotype $(ð)$}

Body elongate, rather flat (Fig. $1 \mathrm{E}) ; \mathrm{LB}=2.81 \mathrm{~mm}$; maximum pronotal width at base $(\mathrm{WP}=0.85 \mathrm{~mm})$; maximum elytral width at basal third $(\mathrm{WE}=1.18 \mathrm{~mm})$. Dorsum dark metallic green. Frons and vertex wrinkled and densely punctate; frontal grooves moderately impressed, not continued to postantennal region; genae and frontal carina moderately elongate; antennae clearly longer than $1 / 2$ body length $(\mathrm{LAN}=2.18 \mathrm{~mm} ; \mathrm{LAN} / \mathrm{LB}=0.77)$, brown; LA: 100:61:69:69:92:100:108:108:108:100:131. Pronotum slightly trapezoidal, moderately transverse $(\mathrm{LP}=0.54 \mathrm{~mm}$; WP/LP $=1.58)$, laterally straight; anterior angles moderately prominent, obliquely beveled; antebasal transverse sulcus clearly impressed, almost straight; lateral fovea clearly distinguishable; pronotal punctuation small, dense, distinctly impressed, evenly distributed on the microreticulate surface. Elytra distinctly elongate $(\mathrm{LE}=2.03 \mathrm{~mm}$; WE/LE $=$ 0.58), laterally slightly arcuate; punctuation clearly larger than on pronotum, strongly impressed; interstriae smooth, very finely microreticulate and micropunctuate. Legs light brown, with dark femora; first pro- and mesotarsomeres distinctly dilated; adhesive setae on ventral side of first pro-, meso- and, partially, metatarsomeres. Venter black; last abdominal ventrite laterally clearly incised, without special preapical impressions. Median lobe of aedeagus ( $\mathrm{LAED}=1.40 \mathrm{~mm} ; \mathrm{LE} / \mathrm{LAED}=1.45)$ (Fig. 8F) slender, in ventral view laterally parallel, slightly narrower in apical part; apex triangular, with a small rounded median tooth; ventral surface with a wide, moderately deep ventral sulcus along central third; in lateral view, median lobe clearly and evenly curved; dorsal ligula as long as half of median lobe, wide but gradually narrower toward apex.

\section{Biometry}

Male ( $\mathrm{n}=3$; range): $2.03 \leq \mathrm{LE} \leq 2.20 \mathrm{~mm} ; 1.18 \leq \mathrm{WE} \leq 1.33 \mathrm{~mm} ; 0.54 \leq \mathrm{LP} \leq 0.64 \mathrm{~mm} ; 0.85 \leq \mathrm{WP} \leq$ $0.93 \mathrm{~mm} ; 2.18 \leq \mathrm{LAN} \leq 2.30 \mathrm{~mm} ; 1.36 \leq \mathrm{LAED} \leq 1.44 \mathrm{~mm} ; 2.81 \leq \mathrm{LB} \leq 3.13 \mathrm{~mm} ; 3.45 \leq \mathrm{LE} / \mathrm{LP} \leq$ $3.77 ; 1.38 \leq \mathrm{WE} / \mathrm{WP} \leq 1.43 ; 1.45 \leq \mathrm{WP} / \mathrm{LP} \leq 1.58 ; 0.58 \leq \mathrm{WE} / \mathrm{LE} \leq 0.60 ; 0.73 \leq \mathrm{LAN} / \mathrm{LB} \leq 0.77 ; 1.45 \leq$ $\mathrm{LE} / \mathrm{LAED} \leq 1.58$.

Female ( $\mathrm{n}=5$; mean and standard deviation; range): $\mathrm{LE}=2.24 \pm 0.07 \mathrm{~mm}(2.15 \leq \mathrm{LE} \leq 2.35 \mathrm{~mm}) ; \mathrm{WE}=$ $1.32 \pm 0.05 \mathrm{~mm}(1.25 \leq \mathrm{WE} \leq 1.38 \mathrm{~mm}) ; \mathrm{LP}=0.62 \pm 0.02 \mathrm{~mm}(0.60 \leq \mathrm{LP} \leq 0.65 \mathrm{~mm}) ; \mathrm{WP}=0.93 \pm$ $0.04 \mathrm{~mm}(0.88 \leq \mathrm{WP} \leq 0.98 \mathrm{~mm}) ; \mathrm{LAN}=1.86 \pm 0.06 \mathrm{~mm}(1.78 \leq \mathrm{LAN} \leq 1.93 \mathrm{~mm}) ; \mathrm{LSPc}=0.20 \pm$ $0.01 \mathrm{~mm}(0.18 \leq \mathrm{LSPc} \leq 0.21 \mathrm{~mm}) ; \mathrm{LB}=3.17 \pm 0.13 \mathrm{~mm}(3.00 \leq \mathrm{LB} \leq 3.34 \mathrm{~mm}) ; \mathrm{LE} / \mathrm{LP}=3.61 \pm 0.04$ $(3.58 \leq \mathrm{LE} / \mathrm{LP} \leq 3.67) ; \mathrm{WE} / \mathrm{WP}=1.41 \pm 0.04(1.36 \leq \mathrm{WE} / \mathrm{WP} \leq 1.46) ; \mathrm{WP} / \mathrm{LP}=1.50 \pm 0.03(1.46 \leq \mathrm{WP} /$ $\mathrm{LP} \leq 1.53) ; \mathrm{WE} / \mathrm{LE}=0.59 \pm 0.01(0.58 \leq \mathrm{WE} / \mathrm{LE} \leq 0.60) ; \mathrm{LAN} / \mathrm{LB}=0.59 \pm 0.01(0.57 \leq \mathrm{LAN} / \mathrm{LB} \leq$ $0.61) ; \mathrm{LE} / \mathrm{LSPc}=11.30 \pm 0.70(10.75 \leq \mathrm{LE} / \mathrm{LSPc} \leq 12.50)$.

Paratypes very similar in shape, sculpture and color to the holotype. Females with shorter antennae. Spermathecal capsule (Fig. 11E) with globose basal part; collum about twice as long as apical part; ductus thin, short, apically inserted. 


\section{Distribution}

Endemic to Mt Humboldt, Southern Grande Terre (Fig. 15A).

\section{Ecological notes}

Collected in montane maquis at $1400 \mathrm{~m}$ a.s.l. No information is available about host plants.

Arsipoda evax Samuelson, 1973

Figs 1F, 8G, 11F, 14A

Arsipoda evax Samuelson, 1973: 128.

Arsipoda species D - Samuelson 1989: 410.

Arsipoda sp. - Samuelson 1994: 181.

\section{Type material examined}

\section{Holotype}

NEW CALEDONIA: §’, Thio, [-21,60682 166,21460], Mar. 1959, N.L.H. Krauss leg. (BPBM);

\section{Paratypes}

NEW CALEDONIA: $1 \hat{\jmath}, 1$, same data as the holotype (BPBM); 1 q, Mokoue to Dothio, $[-21,57642$ 166,13031], 150-200 m, 20-22 Mar. 1968, J.L. Gressitt leg. (BMNH). .

\section{New material}

NEW CALEDONIA: 1 specimen, Koghi Mts, -22.18015 166.51131, 600 m (La Roussette) sifting litter, 27 Oct. 2004, M. Wanat leg. (MNHW); 22 specimens, Chagrin (1 km N of), 20²8.6' S 164 $15.6^{\prime} \mathrm{E}$, $80 \mathrm{~m}$, maquis, 9 Jan. 2007, M. Wanat and R. Dobosz leg. (MNHW); 1 specimen, Baie de Port Bouquet, $21^{\circ} 41.4^{\prime}$ S $166^{\circ} 21.6^{\prime}$ E, 5 m, seashore forest, 2 Jan. 2007, M. Wanat and R. Dobosz leg. (MNHW); 5 specimens, Pic d'Amoa, 2056.1' S 165'17.7, 220-350 m, maquis, 15 Jan. 2007, M. Wanat and R. Dobosz leg. (MNHW); 1 specimen, ditto, 160-200 m, 14 Jan. 2007 (MNHW); 2 specimens, ditto, R. Dobosz and M. Wanat leg. (USMB); 1 specimen, ditto (Povila), -20.93580 165.29520, 220-300 m, maquis, 19 Nov. 2010, M. Wanat and R. Ruta leg. (MNHW); 13 specimens, 8 km SE Nakety, [-21.60889 166.01146], 400 m, on flowers of Mangifera indica L., 22 Sep. 1979, G.A. Samuelson leg. (BPBM); 1 specimen, Col d'Amieu, [-21.61667 165.80000], 500-600 m, 28 Dec. 1976 (BPBM); 3 specimens, Wara, [-21.26667 165.60000], 100 m, on Acacia, 6 Apr. 1981, J.L. Gressitt leg. (BPBM).

\section{Taxonomic notes}

Arsipoda evax shares a thickset, ovoid body with A. atra sp. nov., A. povilaensis sp. nov. and A. transversa sp. nov. (Figs 1B, F, 2D, I). It can be easily distinguished by the smaller size and the black, metallic integuments, (A. atra sp. nov. is black, but not metallic; A. povilaensis sp. nov. is yellowish; A. transversa sp. nov. is reddish). Genitalia are also clearly distinguishable (Figs 8C, G, 9E, 10A, 11B, F, L, P).

Median lobe of aedeagus (Fig. 8G) in ventral view laterally parallel; apex subtriangular, slightly rounded, with generally elongate median tooth; ventral surface with a shallow median depression at apical third; in lateral view median lobe clearly and regularly curved in basal $3 / 4 \mathrm{~S}$ and straight in apical 1/4; dorsal ligula short - from apical third to apical part of median lobe of aedeagus -, wide, apically thinner. Spermatheca (Fig. 11F) with pyriform basal part; collum moderately elongate, about twice as long as apical part; ductus elongate, thickset, apically inserted. 


\section{Biometry}

Male ( $\mathrm{n}=10$; mean and standard deviation; range): $\mathrm{LE}=1.20 \pm 0.09 \mathrm{~mm}(1.10 \leq \mathrm{LE} \leq 1.35 \mathrm{~mm})$; $\mathrm{WE}=$ $0.86 \pm 0.07 \mathrm{~mm}(0.78 \leq \mathrm{WE} \leq 0.98 \mathrm{~mm}) ; \mathrm{LP}=0.38 \pm 0.03 \mathrm{~mm}(0.34 \leq \mathrm{LP} \leq 0.44 \mathrm{~mm}) ; \mathrm{WP}=0.64 \pm$ $0.06 \mathrm{~mm}(0.56 \leq \mathrm{WP} \leq 0.73 \mathrm{~mm}) ; \mathrm{LAN}=0.88 \pm 0.11 \mathrm{~mm}(0.75 \leq \mathrm{LAN} \leq 1.08 \mathrm{~mm}) ; \mathrm{LAED}=0.65 \pm$ $0.04 \mathrm{~mm}(0.60 \leq \mathrm{LAED} \leq 0.71 \mathrm{~mm}) ; \mathrm{LB}=1.63 \pm 0.15 \mathrm{~mm}(1.44 \leq \mathrm{LB} \leq 1.91 \mathrm{~mm}) ; \mathrm{LE} / \mathrm{LP}=3.19 \pm 0.13$ $(3.00 \leq \mathrm{LE} / \mathrm{LP} \leq 3.38) ; \mathrm{WE} / \mathrm{WP}=1.35 \pm 0.03(1.31 \leq \mathrm{WE} / \mathrm{WP} \leq 1.40) ; \mathrm{WP} / \mathrm{LP}=1.69 \pm 0.06(1.61 \leq \mathrm{WP} /$ $\mathrm{LP} \leq 1.81) ; \mathrm{WE} / \mathrm{LE}=0.72 \pm 0.02(0.70 \leq \mathrm{WE} / \mathrm{LE} \leq 0.75) ; \mathrm{LAN} / \mathrm{LB}=0.54 \pm 0.04(0.49 \leq \mathrm{LAN} / \mathrm{LB} \leq 0.60)$; $\mathrm{LE} / \mathrm{LAED}=1.84 \pm 0.05(1.77 \leq \mathrm{LE} / \mathrm{LAED} \leq 1.94)$.

Female ( $\mathrm{n}=10$; mean and standard deviation; range): $\mathrm{LE}=1.28 \pm 0.11 \mathrm{~mm}(1.13 \leq \mathrm{LE} \leq 1.43 \mathrm{~mm})$; $\mathrm{WE}=$ $0.90 \pm 0.10 \mathrm{~mm}(0.75 \leq \mathrm{WE} \leq 1.05 \mathrm{~mm}) ; \mathrm{LP}=0.37 \pm 0.04 \mathrm{~mm}(0.33 \leq \mathrm{LP} \leq 0.41 \mathrm{~mm}) ; \mathrm{WP}=0.65 \pm$ $0.05 \mathrm{~mm}(0.56 \leq \mathrm{WP} \leq 0.73 \mathrm{~mm}) ; \mathrm{LAN}=0.86 \pm 0.09 \mathrm{~mm}(0.73 \leq \mathrm{LAN} \leq 0.98 \mathrm{~mm}) ; \mathrm{LSPc}=0.14 \pm$ $0.02 \mathrm{~mm}(0.10 \leq \mathrm{LSPc} \leq 0.17 \mathrm{~mm}) ; \mathrm{LB}=1.70 \pm 0.14 \mathrm{~mm}(1.53 \leq \mathrm{LB} \leq 1.84 \mathrm{~mm}) ; \mathrm{LE} / \mathrm{LP}=3.45 \pm 0.08$ $(3.25 \leq \mathrm{LE} / \mathrm{LP} \leq 3.54) ; \mathrm{WE} / \mathrm{WP}=1.38 \pm 0.05(1.25 \leq \mathrm{WE} / \mathrm{WP} \leq 1.45) ; \mathrm{WP} / \mathrm{LP}=1.75 \pm 0.06(1.63 \leq \mathrm{WP} /$ $\mathrm{LP} \leq 1.86) ; \mathrm{WE} / \mathrm{LE}=0.70 \pm 0.02(0.65 \leq \mathrm{WE} / \mathrm{LE} \leq 0.74) ; \mathrm{LAN} / \mathrm{LB}=0.51 \pm 0.02(0.47 \leq \mathrm{LAN} / \mathrm{LB} \leq 0.53) ;$ $\mathrm{LE} / \mathrm{LSPc}=9.22 \pm 0.86(8.28 \leq \mathrm{LE} / \mathrm{LSPc} \leq 11.06)$.

\section{Distribution}

Widespread in Northern, Central and Southern Grande Terre (Fig. 14A).

\section{Ecological notes}

Collected in seashore forest and maquis from the coast to $600 \mathrm{~m}$ a.s.1. Found on flowers of Mangifera indica (Anacardiaceae), Acacia sp. (Fabaceae), Styphelia sp. (Ericaceae), Grevillea gillivrayi Hook. (Proteaceae). Pollen found in the gut of some specimens.
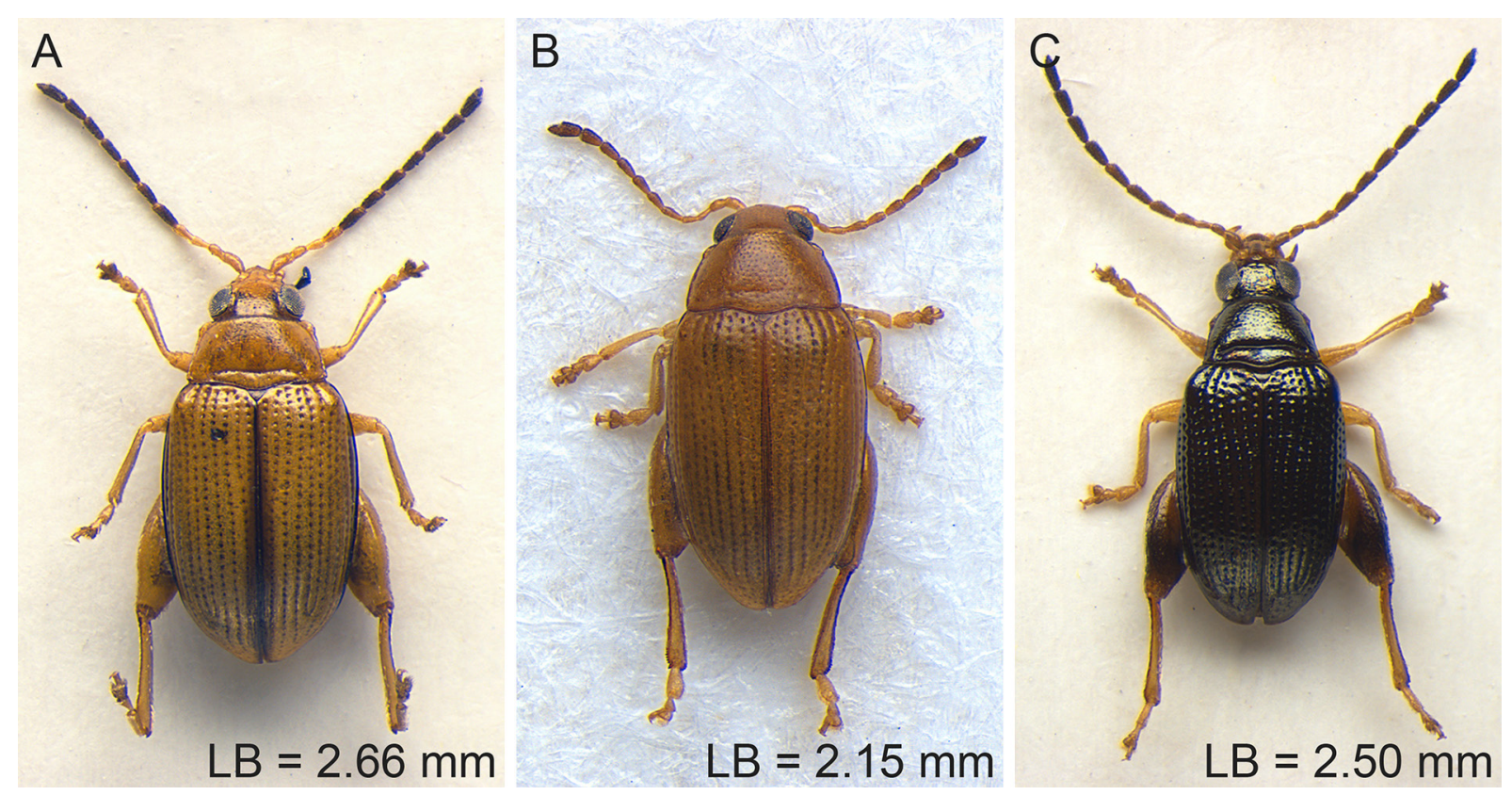

Fig. 3. Habitus. A. Arsipoda wanati sp. nov, Mt Humboldt. B. A. yiambiae Samuelson, 1973, Yiambi. C. A. gomezzuritai sp. nov., Dzumac Mts. 
Arsipoda geographica Gómez-Zurita, 2010

Figs $1 \mathrm{G}, 6 \mathrm{~B}, 8 \mathrm{H}, 11 \mathrm{G}, 14 \mathrm{~A}$

Arsipoda geographica Gómez-Zurita in Gómez-Zurita et al., 2010: 2564.

Arsipoda species A - Samuelson 1989: 409.

Arsipoda sp. - Samuelson 1994: 181.

\section{New material}

NEW CALEDONIA: 1 specimen, Mt Humboldt, -21.87930 166.42320, 1500-1618 m, montane maquis, 13 Nov. 2010, M. Wanat and R. Ruta leg. (MNHW); 11 specimens, ditto, a $1400 \mathrm{~m}$ (MNHW); 1 specimen, ditto, -21.88120 166.41770, 1400-1500 m (MNHW); 2 specimens, ditto, -21.88210 166.41290, $1400 \mathrm{~m}$, night beating, mont. maquis (MNHW); 4 specimens, ditto (S track), Botanical Reserve, -21.88267 166.39968, 1130-1280 m, moss forest, 10 Nov. 2008, M. Wanat leg. (MNHW); 1 specimen, Haute Rivière Bleue, track La Tranchée- $\mathrm{H}^{\text {te }}$ Pourina, $22^{\circ} 04.0^{\prime} \mathrm{S} 166^{\circ} 37.4^{\prime} \mathrm{E}, 330-560 \mathrm{~m}$, 21 Dec. 2006, M. Wanat leg. (MNHW); 1 specimen, Dzumac Mts (Mt Ouin road junction), 22 $01.9^{\prime}$ S 166²8.0' E, 900 m, beating, 28 Dec. 2006, R. Dobosz and M. Wanat leg. (USMB); 1 specimen, ditto (base), $\mathrm{km}$ 1.5-3 E of Ouin rd jct [= road junction], -22.03710 166.49570, $800 \mathrm{~m}$, rainforest, 6 Dec. 2010, R. Ruta and M. Wanat leg. (USMB); 2 specimens, Mt Ouin Rd, km 0-0.5 N of Dzumac jct, -22.03180 166.46740, 900 m, 4 Dec. 2010, R. Ruta and M. Wanat leg. (USMB); 5 specimens, Montagne des Sources [-22.12227 166.59613], 650-700 m, gallery forest, on Zygogynum bicolor Van Tiegh. flowers, 24 Nov. 1976, L.B. Thien leg. (BPBM).

\section{Taxonomic notes}

Arsipoda geographica is, along with $A$. gressitti sp. nov. and A. shirleyae, one of the yellowish species lacking external morphological characters that allow a reliable identification, even though $A$. geographica generally displays elytral interstriae slightly more convex (Fig. 6B); pronotal antebasal sulcus more deeply impressed (Fig. 6B); elytral suture and lateral margin often darkened (Fig. 1G). These species can be distinguished by relying on the morphology of genitalia (Figs 8H-I, 9I, 11G-H, O).

Median lobe of aedeagus (Fig. 8H) in ventral view thickset, laterally parallel, apically widely rounded, with a thin median tooth laterally parallel and apically truncate; ventral surface with a deep median groove in the middle third; in lateral view median lobe distinctly curved; dorsal ligula short, from half length to pre-apical part of median lobe of aedeagus, triangular, with wide base. Spermatheca (Fig. 11G) with large, pyriform basal part; collum elongate, thickset and wrinkled; apical part distinctly shorter than collum; ductus short, thin, apically inserted.

\section{Biometry}

Male ( $\mathrm{n}=10$; mean and standard deviation; range): $\mathrm{LE}=1.96 \pm 0.09 \mathrm{~mm}(1.85 \leq \mathrm{LE} \leq 2.10 \mathrm{~mm}) ; \mathrm{WE}=$ $1.28 \pm 0.07 \mathrm{~mm}(1.18 \leq \mathrm{WE} \leq 1.40 \mathrm{~mm}) ; \mathrm{LP}=0.58 \pm 0.04 \mathrm{~mm}(0.54 \leq \mathrm{LP} \leq 0.65 \mathrm{~mm}) ; \mathrm{WP}=0.93 \pm$ $0.05 \mathrm{~mm}(0.85 \leq \mathrm{WP} \leq 1.03 \mathrm{~mm}) ; \mathrm{LAN}=1.71 \pm 0.08 \mathrm{~mm}(1.60 \leq \mathrm{LAN} \leq 1.83 \mathrm{~mm}) ; \mathrm{LAED}=1.03 \pm$ $0.03 \mathrm{~mm}(0.99 \leq \mathrm{LAED} \leq 1.09 \mathrm{~mm}) ; \mathrm{LB}=2.77 \pm 0.13 \mathrm{~mm}(2.56 \leq \mathrm{LB} \leq 2.94 \mathrm{~mm}) ; \mathrm{LE} / \mathrm{LP}=3.38 \pm 0.15$ $(3.13 \leq \mathrm{LE} / \mathrm{LP} \leq 3.68) ; \mathrm{WE} / \mathrm{WP}=1.38 \pm 0.03(1.34 \leq \mathrm{WE} / \mathrm{WP} \leq 1.45) ; \mathrm{WP} / \mathrm{LP}=1.60 \pm 0.08(1.46 \leq \mathrm{WP} /$ $\mathrm{LP} \leq 1.75) ; \mathrm{WE} / \mathrm{LE}=0.65 \pm 0.02(0.63 \leq \mathrm{WE} / \mathrm{LE} \leq 0.67) ; \mathrm{LAN} / \mathrm{LB}=0.62 \pm 0.02(0.58 \leq \mathrm{LAN} / \mathrm{LB} \leq$ $0.64) ; \mathrm{LE} / \mathrm{LAED}=1.91 \pm 0.05(1.84 \leq \mathrm{LE} / \mathrm{LAED} \leq 1.99)$.

Female ( $\mathrm{n}=10$; mean and standard deviation; range): $\mathrm{LE}=2.02 \pm 0.06 \mathrm{~mm}(1.95 \leq \mathrm{LE} \leq 2.15 \mathrm{~mm})$; $\mathrm{WE}=1.33 \pm 0.07 \mathrm{~mm}(1.28 \leq \mathrm{WE} \leq 1.48 \mathrm{~mm}) ; \mathrm{LP}=0.59 \pm 0.04 \mathrm{~mm}(0.55 \leq \mathrm{LP} \leq 0.68 \mathrm{~mm}) ; \mathrm{WP}=$ $0.95 \pm 0.04 \mathrm{~mm}(0.90 \leq \mathrm{WP} \leq 1.05 \mathrm{~mm}) ; \mathrm{LAN}=1.54 \pm 0.06 \mathrm{~mm}(1.48 \leq \mathrm{LAN} \leq 1.68 \mathrm{~mm}) ; \mathrm{LSPc}=$ $0.20 \pm 0.01 \mathrm{~mm}(0.19 \leq \mathrm{LSPc} \leq 0.22 \mathrm{~mm}) ; \mathrm{LB}=2.82 \pm 0.15 \mathrm{~mm}(2.69 \leq \mathrm{LB} \leq 3.16 \mathrm{~mm}) ; \mathrm{LE} / \mathrm{LP}=$ 
$3.42 \pm 0.12(3.19 \leq \mathrm{LE} / \mathrm{LP} \leq 3.59) ; \mathrm{WE} / \mathrm{WP}=1.39 \pm 0.03(1.32 \leq \mathrm{WE} / \mathrm{WP} \leq 1.44) ; \mathrm{WP} / \mathrm{LP}=1.62 \pm 0.06$ $(1.56 \leq \mathrm{WP} / \mathrm{LP} \leq 1.71) ; \mathrm{WE} / \mathrm{LE}=0.66 \pm 0.02(0.64 \leq \mathrm{WE} / \mathrm{LE} \leq 0.69) ; \mathrm{LAN} / \mathrm{LB}=0.55 \pm 0.02(0.53 \leq$ $\mathrm{LAN} / \mathrm{LB} \leq 0.59) ; \mathrm{LE} / \mathrm{LSPc}=9.93 \pm 0.38(9.32 \leq \mathrm{LE} / \mathrm{LSPc} \leq 10.61)$.

\section{Distribution}

Southern Grande Terre (Fig. 14A).

\section{Ecological notes}

Collected in montane maquis, moss forest, rainforest, and gallery forest, mostly from 800 to $1400 \mathrm{~m}$ a.s.l. Found on flowers of Zygogynum bicolor (Winteraceae). Gómez-Zurita et al. (2010) report molecular evidence associating this species with the genus Ardisia (Myrsinaceae; following the APG III Classification, this family is now included within the Primulaceae). Pollen found in the gut of some specimens.

Arsipoda gomezzuritai sp. nov.

urn:1sid:zoobank.org:act:51AFB32A-32D2-4117-98E2-5E9EFBF6B926

Figs $3 \mathrm{C}, 10 \mathrm{D}, 11 \mathrm{~S}, 15 \mathrm{~B}$

\section{Diagnosis}

This species is similar in shape, sculpture and often color to A. longifrons sp. nov. and A. rostrata (Figs 2A, F, 3C). They all are distinguishable from the other Arsipoda species by the elongate antennae (LAN/LB: $\delta \geq 0.70$, q generally $\geq 0.60$ ) and the elongate frons with narrow frontal carina. The three species can be reliably distinguished by the shape of their genitalia (Figs 9B, G, 10D, 11J, M, S).

\section{Etymology}

Male patronym. Named after Dr. Jesús Gómez-Zurita (Institut de Biologia Evolutiva, Barcelona, Spain) for his contribution to the knowledge of Arsipoda in New Caledonia.

\section{Type material}

\section{Holotype}

NEW CALEDONIA (S): ð̊, Col des Deux Tétons, -22.2059 166.6797, 220-250 m, humid forest, 9 Dec. 2010, M. Wanat \& R. Ruta leg. (MNHN).

\section{Paratypes}

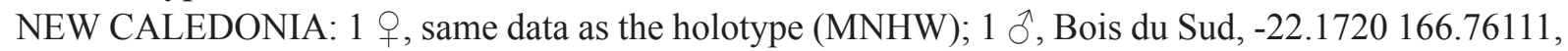
220 m, maquis, 4 Dec. 2008, M. Wanat leg. (MNHW); 1 o, ditto, $22^{\circ} 10.5^{\prime} \mathrm{S} 166^{\circ} 45.8^{\prime} \mathrm{E}, 160 \mathrm{~m}$, maquis, night coll. (lamp and beating), 23 Dec. 2006, R. Dobosz and M. Wanat leg. (USMB); 3 $\widehat{\delta}$, 5 우오, Dzumac Mts (Mt Ouin road junction), 22 $2^{\circ} 01.9^{\prime} \mathrm{S} 166^{\circ} 28.0^{\prime} \mathrm{E}, 900 \mathrm{~m}$, night collecting, 28 Dec. 2006, M. Wanat and R. Dobosz leg. (MNHW); 1 $\hat{\text { }}$, ditto, beating (MNHW); 3 q $q$, ditto (USMB); 1 +, Dzumac Road, $22^{\circ} 06.4^{\prime} \mathrm{S} 166^{\circ} 27.4^{\prime} \mathrm{E}, 470 \mathrm{~m}$, maquis, 30 Dec. 2006, R. Dobosz and M. Wanat leg. (USMB); 2 우, ditto (Mt Ouin to Mts Couvélé road junctions), $22^{\circ} 04.1^{\prime} \mathrm{S} 166^{\circ} 26.8^{\prime} \mathrm{E}, 800$

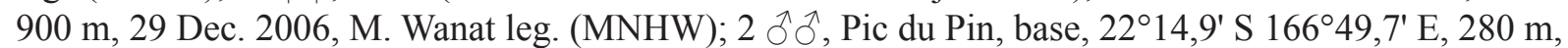
forest and plantation, 25 Dec. 2006, M. Wanat and R. Dobosz leg. (MNHW); 1 đ, 1 q, ditto, -22.24843 166.82883, $280 \mathrm{~m}$, beating, forest edge, 2 Dec. 2008, M. Wanat leg. (MNHW); $2 \hat{\partial} \sigma^{\lambda}$, Haute Rivière

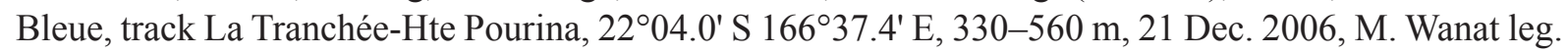
(MNHW); 1 đ̊, 3 우, ditto, track to La Tranchée, $22^{\circ} 05^{\prime} \mathrm{S} 166^{\circ} 38^{\prime} \mathrm{E}, 190-330 \mathrm{~m}, 20 \mathrm{Dec} .2006, \mathrm{M}$. Wanat and R. Dobosz leg. (MNHW); 3 우, Mt Ouin Rd, $400 \mathrm{~m} \mathrm{~N}$ of Dzumac jct, -22.0288 166.4698, $900 \mathrm{~m}$, at light, 6 Dec. 2010, M. Wanat and R. Ruta leg. (MNHW); $2 \widehat{\jmath} \widehat{\partial}, 4$ $\phi$ , ditto, km 0-0.5 N of Dzumac jct, -22.03180 166.46740, 900 m, 4 Dec. 2010, R. Ruta and M. Wanat leg. (MNHW); 2 §ో, ditto, night beating (MNHW). 


\section{Description of the holotype $(\stackrel{\Im}{)})$}

Body moderately elongate and convex; $\mathrm{LB}=2.50 \mathrm{~mm}$; maximum pronotal width at base (WP = $0.83 \mathrm{~mm}$ ); maximum elytral width at basal third $(\mathrm{WE}=1.20 \mathrm{~mm}$ ). Dorsum dark bronze, metallic. Frons and vertex irregularly wrinkled and punctate; head grooves distinctly impressed, not continued to postantennal region; genae and frontal carina elongate; antennae clearly longer than $1 / 2$ body length $(\mathrm{LAN}=1.98 \mathrm{~mm} ; \mathrm{LAN} / \mathrm{LB}=0.79)$, basally light brown, gradually and slightly darker toward apex; LA: 100:69:61:92:115:115:131:138:131:123:154. Pronotum slightly trapezoidal, moderately transverse $(\mathrm{LP}=0.54 \mathrm{~mm} ; \mathrm{WP} / \mathrm{LP}=1.53)$, laterally weakly concave; anterior angles not prominent; antebasal transverse sulcus strongly impressed, weakly sinuate; lateral fovea not clearly distinguishable; pronotal punctuation small and shallow, evenly distributed on the irregularly microreticulate and very sparsely micropunctuate surface. Elytra moderately elongate $(\mathrm{LE}=1.88 \mathrm{~mm} ; \mathrm{WE} / \mathrm{LE}=0.64)$, laterally slightly arcuate; punctuation clearly larger than on pronotum, strongly impressed; interstriae smooth, very finely micropunctate. Legs yellow, with darkened femora; first pro- and mesotarsomeres distinctly dilated; adhesive setae present on ventral side of first pro-, meso- and, partially, metatarsomeres. Venter brownish; last abdominal ventrite laterally clearly incised, without special preapical impressions. Median lobe of aedeagus $(\mathrm{LAED}=1.17 \mathrm{~mm}$; LE/LAED $=1.61)($ Fig. $10 \mathrm{D})$ in ventral view thickset, narrower at base and at apical third, and distally widely lanceolate, with median rounded small tooth; ventral surface with deep ventral sulcus; in lateral view, median lobe straight, with two lateral small humps at apical third, and small sinuate apex dorsally bent; dorsal ligula short, from apical third to apical part of median lobe of aedeagus, narrow, thinner toward apex.

\section{Biometry}

Male ( $\mathrm{n}=10$; mean and standard deviation; range): $\mathrm{LE}=2.01 \pm 0.13 \mathrm{~mm}(1.88 \leq \mathrm{LE} \leq 2.23 \mathrm{~mm})$; $\mathrm{WE}=$ $1.31 \pm 0.08 \mathrm{~mm}(1.20 \leq \mathrm{WE} \leq 1.45 \mathrm{~mm}) ; \mathrm{LP}=0.56 \pm 0.03 \mathrm{~mm}(0.53 \leq \mathrm{LP} \leq 0.60 \mathrm{~mm}) ; \mathrm{WP}=0.89 \pm$ $0.05 \mathrm{~mm}(0.83 \leq \mathrm{WP} \leq 0.99 \mathrm{~mm}) ; \mathrm{LAN}=2.12 \pm 0.14 \mathrm{~mm}(1.98 \leq \mathrm{LAN} \leq 2.35 \mathrm{~mm}) ;$ LAED $=1.25 \pm$ $0.09 \mathrm{~mm}(1.16 \leq \mathrm{LAED} \leq 1.38 \mathrm{~mm}) ; \mathrm{LB}=2.69 \pm 0.16 \mathrm{~mm}(2.50 \leq \mathrm{LB} \leq 2.97 \mathrm{~mm}) ; \mathrm{LE} / \mathrm{LP}=3.58 \pm 0.15$ $(3.38 \leq \mathrm{LE} / \mathrm{LP} \leq 3.83) ; \mathrm{WE} / \mathrm{WP}=1.47 \pm 0.05(1.42 \leq \mathrm{WE} / \mathrm{WP} \leq 1.58) ; \mathrm{WP} / \mathrm{LP}=1.58 \pm 0.05(1.50 \leq \mathrm{WP} /$ $\mathrm{LP} \leq 1.65) ; \mathrm{WE} / \mathrm{LE}=0.65 \pm 0.01(0.64 \leq \mathrm{WE} / \mathrm{LE} \leq 0.67) ; \mathrm{LAN} / \mathrm{LB}=0.79 \pm 0.03(0.75 \leq \mathrm{LAN} / \mathrm{LB} \leq$ $0.83) ; \mathrm{LE} / \mathrm{LAED}=1.60 \pm 0.06(1.51 \leq \mathrm{LE} / \mathrm{LAED} \leq 1.70)$.

Female ( $\mathrm{n}=10$; mean and standard deviation; range): $\mathrm{LE}=2.25 \pm 0.15 \mathrm{~mm}(2.03 \leq \mathrm{LE} \leq 2.45 \mathrm{~mm})$; $\mathrm{WE}=1.44 \pm 0.11 \mathrm{~mm}(1.25 \leq \mathrm{WE} \leq 1.55 \mathrm{~mm}) ; \mathrm{LP}=0.62 \pm 0.06 \mathrm{~mm}(0.53 \leq \mathrm{LP} \leq 0.69 \mathrm{~mm}) ; \mathrm{WP}=$ $0.95 \pm 0.08 \mathrm{~mm}(0.83 \leq \mathrm{WP} \leq 1.05 \mathrm{~mm}) ; \mathrm{LAN}=1.87 \pm 0.14 \mathrm{~mm}(1.65 \leq \mathrm{LAN} \leq 2.03 \mathrm{~mm}) ; \mathrm{LSPc}=$ $0.18 \pm 0.01 \mathrm{~mm}(0.16 \leq \mathrm{LSPc} \leq 0.21 \mathrm{~mm}) ; \mathrm{LB}=3.01 \pm 0.20 \mathrm{~mm}(2.69 \leq \mathrm{LB} \leq 3.25 \mathrm{~mm}) ; \mathrm{LE} / \mathrm{LP}=$ $3.63 \pm 0.20(3.30 \leq \mathrm{LE} / \mathrm{LP} \leq 3.88) ; \mathrm{WE} / \mathrm{WP}=1.51 \pm 0.04(1.44 \leq \mathrm{WE} / \mathrm{WP} \leq 1.57) ; \mathrm{WP} / \mathrm{LP}=1.54 \pm 0.04$ $(1.46 \leq \mathrm{WP} / \mathrm{LP} \leq 1.60) ; \mathrm{WE} / \mathrm{LE}=0.64 \pm 0.01(0.62 \leq \mathrm{WE} / \mathrm{LE} \leq 0.66) ; \mathrm{LAN} / \mathrm{LB}=0.62 \pm 0.02(0.57 \leq$ $\mathrm{LAN} / \mathrm{LB} \leq 0.65) ; \mathrm{LE} / \mathrm{LSPc}=12.44 \pm 1.00(11.18 \leq \mathrm{LE} / \mathrm{LSPc} \leq 13.92)$.

Paratypes generally very similar in shape and sculpture to the holotype; some specimens paler. Females with clearly shorter antennae. Spermathecal capsule (Fig. 11S) thin and curved, with elongate and wrinkled collum; basal part slightly enlarged, not abruptly separated from collum; apical part short; ductus short, apically inserted.

\section{Distribution}

Southern Grande Terre (Fig. 15B).

\section{Ecological notes}

Collected in humid forest, maquis and plantation between 160 and $900 \mathrm{~m}$ a.s.l. No information is available about host plants. Pollen found in the gut of some specimens. 


\section{Arsipoda gressitti sp. nov. urn:Isid:zoobank.org:act:75D53499-67B7-4382-A9CF-DB929FE5556C}

Figs $1 \mathrm{H}, 8 \mathrm{I}, 11 \mathrm{H}, 15 \mathrm{~A}$

\section{Diagnosis}

Arsipoda gressitti sp. nov. is, along with A. geographica and A. shirleyae, one of the yellowish species lacking ventral morphological characters that allow a reliable identification. These species can be distinguished only by relying on the morphology of genitalia (Figs 8H-I, 9I, 11G-H, O).

\section{Etymology}

Male patronym. Named after Dr. J. Linsley Gressitt (1914-1982), who devoted his energies to the establishment, development and expansion of the Entomology Department of the Bishop Museum (Honolulu).

\section{Type material}

\section{Holotype}

NEW CALEDONIA: Ō, Plateau de Dogny [-21.60987 165.88907], Melaleuca savanna, 9 Apr. 1973, J.L. Gressitt leg. (BPBM).

\section{Paratypes}

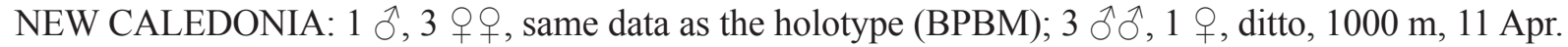

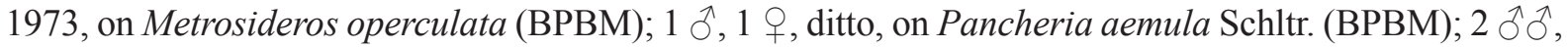

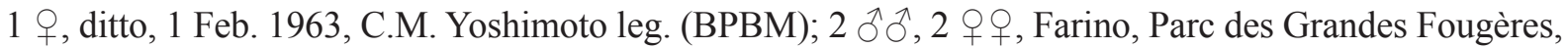
N of Pic Vincent, -21.59929 165.77519, 670 m, track and forest, 17 Nov. 2008, M. Wanat leg.(MNHW);

\section{Description of the holotype $(\circlearrowleft)$}

Body moderately elongate, feebly convex; $\mathrm{LB}=2.22 \mathrm{~mm}$; maximum pronotal width at base $(\mathrm{WP}=0.71 \mathrm{~mm})$; maximum elytral width at apical third $(\mathrm{WE}=0.98 \mathrm{~mm})$. Dorsum yellowish, metallic, with irregular darkened spots on elytra. Frons and vertex microreticulate and punctate; head grooves weakly impressed, not continued to postantennal region; genae and frontal carina short; antennae slightly longer than $1 / 2$ body length $(\mathrm{LAN}=1.30 \mathrm{~mm}$; LAN/LB $=0.59)$, basally yellow, gradually and slightly darker toward apex; LA: 100:67:67:54:75:75:92:87:92:92:125. Pronotum trapezoidal, distinctly transverse $(\mathrm{LP}=0.45 \mathrm{~mm}$; WP/LP $=1.58)$, laterally straight; anterior angles moderately prominent; antebasal transverse sulcus moderately impressed, slightly sinuate, with small lateral fovea; pronotal surface microreticulate, with small, dense punctures, distinctly impressed. Elytra moderately elongate $(\mathrm{LE}=1.55 \mathrm{~mm} ; \mathrm{WE} / \mathrm{LE}=0.63)$, laterally moderately arcuate; punctuation clearly larger than on pronotum and clearly impressed; interstriae microreticulate. Legs yellowish; first pro- and mesotarsomeres weakly dilated; adhesive setae present on ventral side of first pro-, meso- and, partially, metatarsomeres. Venter light brown; last abdominal ventrite laterally incised, without special preapical impressions. Median lobe of aedeagus $(\mathrm{LAED}=0.90 \mathrm{~mm}$; LE/LAED $=1.71)$ (Fig. 8I) in ventral view fusiform, but narrower in preapical part; apex with median rounded tooth; ventral surface broadly concave at basal half; in lateral view, median lobe curved at basal third and in preapical part, thin especially at basal third, with straight apex; dorsal ligula from about half length to preapical part of median lobe of aedeagus, wide, tapered toward apex.

\section{Biometry}

Male ( $\mathrm{n}=10$; mean and standard deviation; range): $\mathrm{LE}=1.72 \pm 0.13 \mathrm{~mm}(1.55 \leq \mathrm{LE} \leq 1.94 \mathrm{~mm})$; $\mathrm{WE}=$ $1.09 \pm 0.09 \mathrm{~mm}(0.98 \leq \mathrm{WE} \leq 1.24 \mathrm{~mm}) ; \mathrm{LP}=0.51 \pm 0.04 \mathrm{~mm}(0.45 \leq \mathrm{LP} \leq 0.56 \mathrm{~mm}) ; \mathrm{WP}=0.82 \pm$ $0.07 \mathrm{~mm}(0.71 \leq \mathrm{WP} \leq 0.92 \mathrm{~mm}) ; \mathrm{LAN}=1.46 \pm 0.10 \mathrm{~mm}(1.36 \leq \mathrm{LAN} \leq 1.60 \mathrm{~mm}) ; \mathrm{LAED}=0.99 \pm$ 
$0.05 \mathrm{~mm}(0.90 \leq \mathrm{LAED} \leq 1.06 \mathrm{~mm}) ; \mathrm{LB}=2.30 \pm 0.10 \mathrm{~mm}(2.13 \leq \mathrm{LB} \leq 2.40 \mathrm{~mm}) ; \mathrm{LE} / \mathrm{LP}=3.39 \pm 0.06$ $(3.32 \leq \mathrm{LE} / \mathrm{LP} \leq 3.49) ; \mathrm{WE} / \mathrm{WP}=1.33 \pm 0.03(1.29 \leq \mathrm{WE} / \mathrm{WP} \leq 1.37) ; \mathrm{WP} / \mathrm{LP}=1.61 \pm 0.02(1.58 \leq \mathrm{WP} /$ $\mathrm{LP} \leq 1.64) ; \mathrm{WE} / \mathrm{LE}=0.63 \pm 0.01(0.61 \leq \mathrm{WE} / \mathrm{LE} \leq 0.65) ; \mathrm{LAN} / \mathrm{LB}=0.63 \pm 0.02(0.59 \leq \mathrm{LAN} / \mathrm{LB} \leq$ $0.67) ; \mathrm{LE} / \mathrm{LAED}=1.73 \pm 0.06(1.65 \leq \mathrm{LE} / \mathrm{LAED} \leq 1.85)$.

Female ( $\mathrm{n}=8$; mean and standard deviation; range): $\mathrm{LE}=1.90 \pm 0.08 \mathrm{~mm}(1.80 \leq \mathrm{LE} \leq 2.04 \mathrm{~mm}) ; \mathrm{WE}=$ $1.19 \pm 0.04 \mathrm{~mm}(1.14 \leq \mathrm{WE} \leq 1.25 \mathrm{~mm}) ; \mathrm{LP}=0.54 \pm 0.03 \mathrm{~mm}(0.52 \leq \mathrm{LP} \leq 0.58 \mathrm{~mm}) ; \mathrm{WP}=0.89 \pm$ $0.04 \mathrm{~mm}(0.82 \leq \mathrm{WP} \leq 0.94 \mathrm{~mm}) ; \mathrm{LAN}=1.38 \pm 0.05 \mathrm{~mm}(1.30 \leq \mathrm{LAN} \leq 1.44 \mathrm{~mm}) ; \mathrm{LSPc}=0.19 \pm$ $0.01 \mathrm{~mm}(0.17 \leq \mathrm{LSPc} \leq 0.20 \mathrm{~mm}) ; \mathrm{LB}=2.51 \pm 0.10 \mathrm{~mm}(2.35 \leq \mathrm{LB} \leq 2.63 \mathrm{~mm}) ; \mathrm{LE} / \mathrm{LP}=3.53 \pm 0.07$ $(3.41 \leq \mathrm{LE} / \mathrm{LP} \leq 3.62) ; \mathrm{WE} / \mathrm{WP}=1.35 \pm 0.04(1.30 \leq \mathrm{WE} / \mathrm{WP} \leq 1.41) ; \mathrm{WP} / \mathrm{LP}=1.64 \pm 0.05(1.58 \leq \mathrm{WP} /$ $\mathrm{LP} \leq 1.73) ; \mathrm{WE} / \mathrm{LE}=0.63 \pm 0.02(0.61 \leq \mathrm{WE} / \mathrm{LE} \leq 0.66) ; \mathrm{LAN} / \mathrm{LB}=0.55 \pm 0.01(0.52 \leq \mathrm{LAN} / \mathrm{LB} \leq$ $0.57) ; \mathrm{LE} / \mathrm{LSPc}=10.12 \pm 0.91(9.51 \leq \mathrm{LE} / \mathrm{LSPc} \leq 12.14)$.

Paratypes very similar in shape, sculpture and color to the holotype. Antennae distinctly shorter in female. Spermatheca (Fig. 11H) with globose, slightly elongate basal part, elongate collum, and short apical part; ductus thin, short, apically inserted.

\section{Distribution}

Central Grande Terre (Fig. 15A).

\section{Ecological notes}

Collected in forest and along track between 670 and $1000 \mathrm{~m}$ a.s.1. Found on Pancheria aemula (Cunoniaceae) and Metrosideros operculata (Myrtaceae).

Arsipoda isola Samuelson, 1973

Figs 1I, 4D, 9A, 11I, 14A

Arsipoda isola Samuelson, 1973: 129.

Arsipoda isola - Samuelson 1989: 409; 1994: 181. — Gómez-Zurita et al. 2010: 2560.

Type material examined

Holotype

NEW CALEDONIA: §̂, Plaine des Lacs, [-22,25000 166,91667], 2 Feb. 1963, C.M. Yoshimoto (BPBM);

Paratypes

NEW CALEDONIA: 1 ô, 1 \%, same data as the holotype (BPBM); 1 q, ditto, J.L. Gressitt leg. (BMNH).

\section{New material}

NEW CALEDONIA: 8 specimens, Boulouparis/Mont Do, -21.75394 161.99994 [165.99994], 1029 m, battage, collected on Araucaria laubenfelsii, 6 Oct. 2004, S. Cazères and C. Mille leg., coll. Christian Mille (BAQ); 1 specimen, Mont Do, [-21.7574 166.0015], beating, Araucaria laubenfelsii branches, 21 Nov. 2002, R. Mecke leg., coll. Christian Mille (BAQ); 23 specimens, ditto, -21.7574 166.0015, 850-950 m, night beating, 2 Nov. 2010, M. Wanat and R. Ruta leg. (MNHW); 3 specimens, ditto, $-21.7606165 .9996,850 \mathrm{~m}$, subsummit forest, at light, 2 Nov. 2010 (MNHW); 1 specimen, ditto, $-21.75585166 .00099,900-1025 \mathrm{~m}$, maquis and forest edge, 6 Nov. 2008, M. Wanat leg. (MNHW); 4 specimens, ditto, -21.76674 166.00540, 820-920 m, roadside, night beating, 6 Nov. 2008 (MNHW); 1 specimen, ditto, $850 \mathrm{~m}$, subsummit forest, at light, 5 Nov. 2008 (MNHW); 1 specimen, ditto, -21.75706 165.99894, 920 m, at light, 6 Nov. 2008 (MNHW); 1 specimen, ditto, -21.76527 166.00228, 800-850 m, forest, night beating, 5 Nov. 2008 (MNHW); 1 specimen, ditto (summit), 21 $1^{\circ} 45.1^{\prime} \mathrm{S} 166^{\circ} 00.0^{\prime} \mathrm{E}, 1000$ 
1025 m, 4 Jan. 2007, R. Dobosz and M. Wanat leg. (USMB); 1 specimen, Piste Est Col des Roussettes: PK 10-15, [-21.427953 165.457728], 600 m, battage d'arbustes, 24 Nov. 2002, A. Mantilleri and E.-A. Leguin leg., 977, coll. Christian Mille (BAQ); 3 specimens, Pic du Pin (base), 22¹4.9' S 166 49.7' E, $280 \mathrm{~m}$, night coll. (lamp and beating), 25 Dec. 2006, M. Wanat and R. Dobosz leg. (MNHW); 1 specimen, ditto, forest and plantation (MNHW); 1 specimen, ditto, 26 Dec. 2006 (MNHW); 1 specimen, ditto,
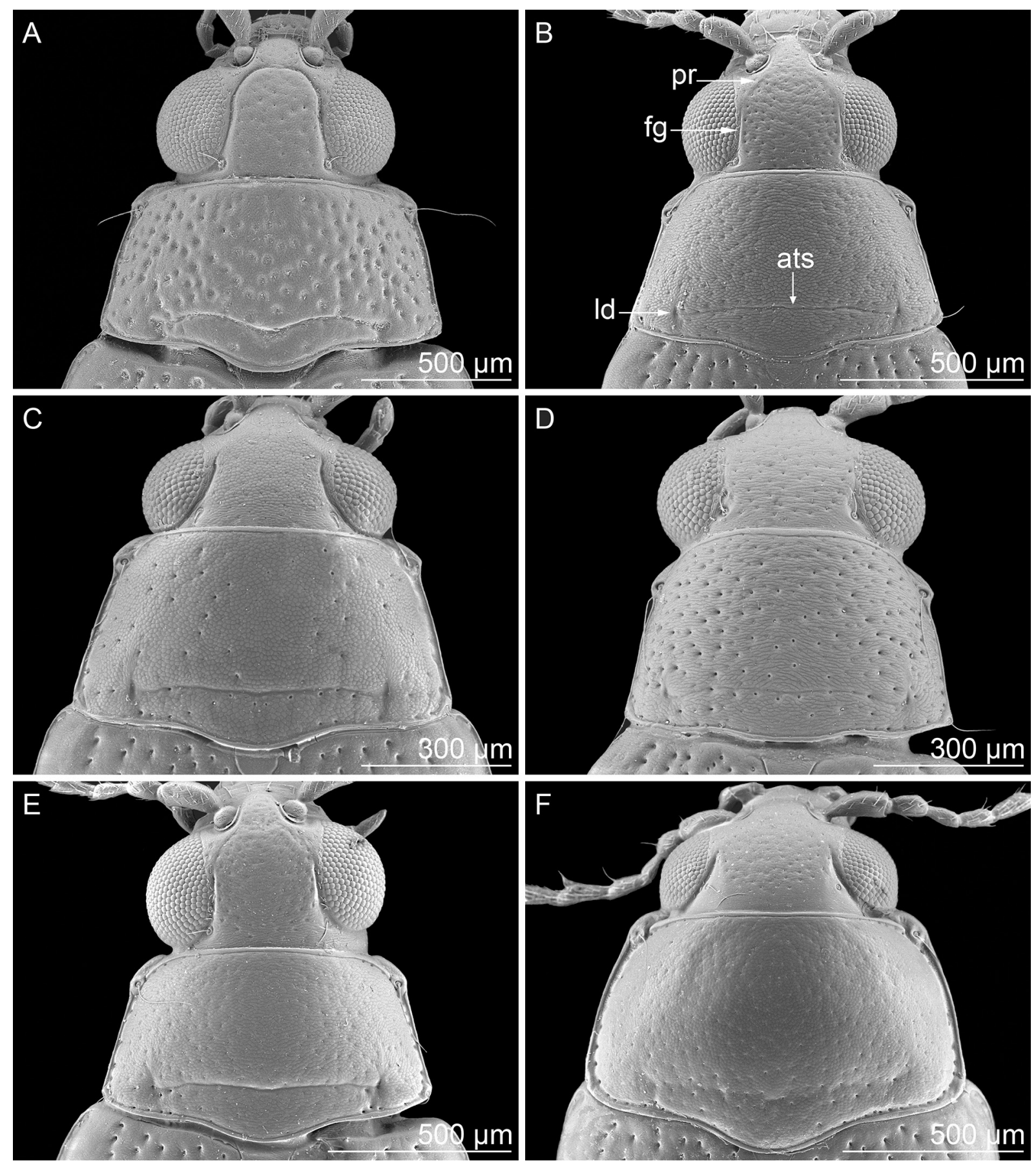

Fig. 4. Head and pronotum. A. Arsipoda agalma Samuelson, 1973, Col d'Amieu. B. A. communis sp. nov., Dzumac Mts. C. A. doboszi sp. nov, Haute Rivière Bleue. D. A. isola Samuelson, 1973, Mont Do. E. A. montana sp. nov., Mt Humboldt. F. A. paniensis sp. nov., Mont Panié. Abbreviations: ats $=$ antebasal transverse sulcus; $\mathrm{fg}=$ frontal grooves; $\mathrm{ld}=$ lateral dimples; $\mathrm{pr}=$ postantennal region. 
-22.24810 166.82920, 280 m, plantation, 31 Oct. 2010, M. Wanat and R. Ruta leg. (MNHW); 2 specimens, ditto, -22.24843 166.82883, 280 m, forest, 22 Oct. 2008, M. Wanat leg. (MNHW); 4 specimens, ditto, at light (MNHW); 2 specimens, ditto, 21 Oct. 2008 (MNHW); 1 specimen, ditto, plantation (MNHW); 1 specimen, Pic du Grand Kaori, 22 ${ }^{\circ} 16.8^{\prime}$ S $166^{\circ} 53.5^{\prime}$ E, 240 m, night coll. (lamp and beating), 26 Dec. 2006, M. Wanat and R. Dobosz leg. (MNHW); 2 specimens, ditto, -22.28430 166.89540, 220 m, night, 1 Nov. 2010, M. Wanat and R. Ruta leg. (MNHW); 1 specimen, ditto (Bot. Reserve), $22^{\circ} 17^{\prime}$ S $166^{\circ} 54^{\prime} \mathrm{E}$, $250 \mathrm{~m}$, humid forest, $15 \mathrm{Feb}$. 2004, M. Wanat leg. (MNHW); 1 specimen, Koghi Mts, -22.17809 166.50569, $470 \mathrm{~m}$, auberge, at light (inside car), 2 Dec. 2008, M. Wanat leg. (MNHW); 1 specimen, ditto, $22^{\circ} 11^{\prime} \mathrm{S} 166^{\circ} 30^{\prime} \mathrm{E}, 500-550 \mathrm{~m}$, humid forest, $21 \mathrm{Jan} .2004$, M. Wanat leg. (MNHW); 1 specimen, ditto, $22^{\circ} 10.7^{\prime} \mathrm{S}, 166^{\circ} 30.4^{\prime} \mathrm{E}, 450-500 \mathrm{~m}$, rainforest, netting, 16 Dec. 2006, R. Dobosz leg. (USMB); 46 specimens, Bois du Sud, $22^{\circ} 10.5^{\prime} \mathrm{S} 166^{\circ} 45.8^{\prime} \mathrm{E}, 160 \mathrm{~m}$, maquis, night coll. (lamp and beating), 23 Dec. 2006, M. Wanat and R. Dobosz leg. (MNHW); 6 specimens, ditto (USMB); 2 specimens, ditto, at light, R. Dobosz leg. (USMB); 7 specimens, ditto, maquis, 24 Dec. 2006 (MNHW); 1 specimen, ditto, -22.17200 166.76111, 220 m, beating, 2 Mar. 2008, R. Dobosz and T. Blaik leg. (MNHW); 3 specimens, ditto, at light, 23 Oct. 2008, M. Wanat leg. (MNHW); 23 specimens, ditto, 17 Oct. 2008 (MNHW); 1 specimen, ditto, 26 Oct. 2008 (MNHW); 2 specimens, ditto, day beating, 18 Oct. 2008 (MNHW); 4 specimens, ditto, maquis, night beating, 20 Oct. 2008 (MNHW); 3 specimens, ditto, -22.17288 166.76330, 220-250 m, beating along track entering forest reserve, 20 Oct. 2008 (MNHW); 3 specimens, ditto, -22.17590 166.76250, 220 m, 10 Dec. 2010, M. Wanat and R. Ruta leg. (MNHW); 1 specimen, ditto ('Araucaria' hut), -22.17400 166.76270, 220 m, at light, 8 Dec. 2010 (MNHW); 1 specimen, Bois du Sud, camp, $22^{\circ} 10.5^{\prime} \mathrm{S} 166^{\circ} 45.8^{\prime} \mathrm{E}, 160 \mathrm{~m}, 23$ Dec. 2006, R. Dobosz leg. (USMB); 1 specimen, ditto, rainforest, at light (USMB); 9 specimens, ditto, $22^{\circ} 10.443^{\prime} \mathrm{S} 166^{\circ} 45.760^{\prime} \mathrm{E}, 210 \mathrm{~m}$, biting, at light, maquis and rainforest, 9 Mar. 2008, R. Dobosz and T. Blaik leg. (USMB); 4 specimens, ditto, at light, rainforest, 23 Mar. 2008 (USMB); 7 specimens, ditto, 10 Apr. 2008 (USMB); 1 specimen, ditto, 9 Apr. 2008 (USMB); 2 specimens, ditto, at light, maquis and rainforest, 3 Mar. 2008 (USMB); 3 specimens, ditto, 1 Mar. 2008 (USMB); 1 specimen, ditto, 2 Mar. 2008 (USMB); 20 specimens, ditto, 5 Mar. 2008 (USMB); 3 specimens, ditto, biting maquis Hibbertia sp., R. Dobosz leg. (USMB); 24 specimens, Mine Gallieni (Tontouta valley), -21.90211 166.34514, $410 \mathrm{~m}$, at light, 8 Nov. 2008, M. Wanat leg. (MNHW); 1 specimen, Tontouta valley, start of Humboldt track, -21.91272 166.35330, 500-530 m, maquis, 2 Nov. 2008, M. Wanat (MNHW); 4 specimens, Chute de la Madeleine, $22^{\circ} 14.2^{\prime}$ S $166^{\circ} 51.7^{\prime}$ E, 240 m, maquis, night coll. (lamp and beating), 24 Dec. 2006. M. Wanat and R. Dobosz leg. (MNHW); 5 specimens, ditto (USMB); 1 specimen, Rivière Bleue Parc, refuge, $22^{\circ} 05.9^{\prime} \mathrm{S} 166^{\circ} 38.3^{\prime} \mathrm{E}, 190 \mathrm{~m}$, night coll. (lamp and beating), 20 Dec. 2006, M. Wanat and R. Dobosz leg. (MNHW); 1 specimen, ditto, at light, R. Dobosz leg. (USMB); 1 specimen, ditto, at light, 19 Dec. 2007, M. Wanat and R. Dobosz leg. (MNHW); 1 specimen, ditto, scient. Refuge, $22^{\circ} 06.0^{\prime} \mathrm{S} 166^{\circ} 38.6^{\prime} \mathrm{E}, 180 \mathrm{~m}$, at light, $21 \mathrm{Jan}$. 2007, M. Wanat and R. Dobosz leg. (MNHW); 3 specimens, ditto, 22 Jan. 2007 (MNHW); 1 specimen, ditto, 190 m (USMB);

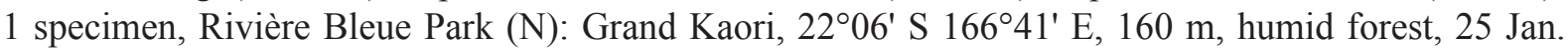
2004, M. Wanat leg. (MNHW); 5 specimens, ditto, 26 Jan. 2004 (MNHW); 2 specimens, Haute Rivière Bleue, $22^{\circ} 05^{\prime} \mathrm{S} 166^{\circ} 38^{\prime}$ E, 180-330 m, track to La Tranchée, 23 Jan. 2004, M. Wanat leg. (MNHW); 2 specimens, Dzumac Road, 22 ${ }^{\circ} 06.4^{\prime}$ S $166^{\circ} 27.4^{\prime}$ E, 470 m, maquis, 30 Dec. 2006, M. Wanat and R. Dobosz leg. (MNHW); 2 specimens, ditto, -22.08783 166.44643, 650-700 m, 1 Feb. 2008, M. Wanat leg. (MNHW); 9 specimens, ditto (Mt Ouin to Mts Couvélé road junctions), 22 04.1' S $166^{\circ} 26.8^{\prime} \mathrm{E}$, 800-900 m, 29 Dec. 2006 (MNHW); 5 specimens, ditto, S of Mts Couvélé rd jct [= road junction], -22.06913 166.44666, 870-670 m, beating, 31 Oct. 2008 (MNHW); 3 specimens, Dzumac Mts, $22^{\circ} 01.9^{\prime} \mathrm{S} 166^{\circ} 28.0^{\prime} \mathrm{E}, 900 \mathrm{~m}$, netting, 28 Dec. 2006, R. Dobosz leg. (USMB); 5 specimens, ditto, Mt Ouin road junction, 30 Dec. 2006 (USMB); 1 specimen, ditto, at light, 29 Dec. 2006 (USMB); 3 specimens, ditto, -22.03188 166.46738, 910 m, 28 Oct. 2008, M. Wanat leg. (MNHW); 2 specimens, ditto, 22 ${ }^{\circ} 01.9^{\prime} \mathrm{S} 166^{\circ} 28.0^{\prime} \mathrm{E}, 900 \mathrm{~m}, 28$ Dec. 2006, M. Wanat and R. Dobosz leg. (MNHW); 2 specimens, Poro Plateau, -21.34830 165.69320, 620 m, forest, 28 Nov. 2010, R. Ruta, M. Wanat leg. (MNHW); 3 specimens, ditto, -21.34832 165.69322, night, 27 Nov. 2010 (MNHW); 1 specimen, Poro (8 km SSE), 
-21.34897 165.68429, $550 \mathrm{~m}$, plateau above mine, ex Hibbertia lucens, 30 Nov. 2008, M. Wanat leg. (MNHW); 1 specimen, Mt Ouin Rd, $400 \mathrm{~m} \mathrm{~N}$ of Dzumac jct, -22.02880 166.46980, $900 \mathrm{~m}$, at light, 6 Dec. 2010, M. Wanat and R. Ruta leg. (MNHW); 1 specimen, track Col des Agathis to Forest Cachee, -22.17288 166.76330, 500-340 m, 26 Oct. 2008, M. Wanat leg. (MNHW); 1 specimen, Foret Nord (Bot. Reserve), 22 $2^{\circ} 19^{\prime} \mathrm{S} 166^{\circ} 55^{\prime} \mathrm{E}, 200 \mathrm{~m}$, maquis and humid forest, 15 Feb. 2004, M. Wanat leg. (MNHW); 1 specimen, Plum, [-22.27410 166.61574], 20-60 m, malaise trap, 23-25 Mar. 1968, J.L. Gressitt and T.C. Maa leg. (BPBM); 3 specimens, between Plum andYate, [-22.16975 166.75320], 25 Mar. 1968, J.L. Gressitt and T.C. Maa leg. (BPBM); 1 specimen, Yate, 5 km ex Fork, 2-50 m, 25 Mar. 1968, J.L. Gressitt and T.C. Maa leg. (BPBM); 1 specimen, Yate [-22.16670 166.95000], 26-27 Mar. 1968, J.L. Gressitt and T.C. Maa leg. (BPBM); 1 specimen, ditto, Sapium, 26 Mar. 1968 (BPBM); 7 specimens, Col de Yate (highest point), -22.16834 166.90129, 380 m, maquis, 2 Dec. 2008, M. Wanat leg. (MNHW); 5 specimens, Yate env., Niüwede Riv., $22^{\circ} 09.758^{\prime} \mathrm{S} 166^{\circ} 55.460^{\prime} \mathrm{E}, 8 \mathrm{~m}$, at light, 2 Mar. 2008, R.

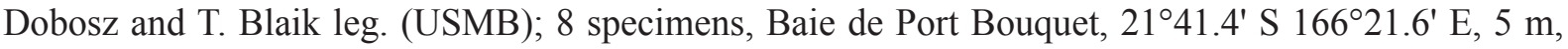
seashore forest, 2 Jan. 2007, M. Wanat and R. Dobosz leg. (MNHW); 3 specimens, S Humboldt trail to Col du Vulcain, -21.90430 166.38100, 800-950 m, maquis, 11 Nov. 2010, M. Wanat and R. Ruta leg. (MNHW); 1 specimen, Mt Humboldt, track La tranchée-Hte Pourina, -21.88120 166.41770, 14001500 m, montane maquis, 13 Nov. 2010, M. Wanat and R. Ruta leg. (MNHW); 2 specimens, Humboldt (S track), -21.90517 166.35621, 800-950 m, maquis, beating down Col du Vulcain, 11 Nov. 2008, M. Wanat leg. (MNHW); 6 specimens, ditto, maquis, beating along track to Col du Vulcain, 9 Nov. 2008 (MNHW); 1 specimen, ditto, Col du Vulcain (refuge), -21.90319 166.38305, 980 m, ex Hibbertia trachyphylla Schltr., 10 Nov. 2008 (MNHW); 1 specimen, ditto, Botanical Reserve, -21.88267 166.39968, 1130-1280 m, moss forest, 10 Nov. 2008 (MNHW); 8 specimens, Nyamié creek, -21.76503 166.42538, 20-40 m, maquis, 4 Nov. 2008, M. Wanat leg. (MNHW); 10 specimens, ditto, at light

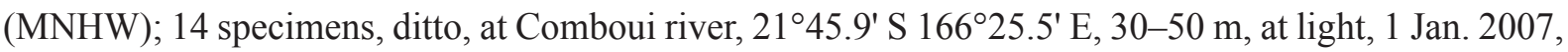
M. Wanat and R. Dobosz leg. (MNHW); 1 specimen, ditto, night collecting (USMB); 66 specimens, ditto, 31 Dec. 2006 (MNHW); 10 specimens, ditto (USMB); 1 specimen, Nouméa (Magenta), $22^{\circ} 15.799^{\prime} \mathrm{S} 166^{\circ} 28.007^{\prime} \mathrm{E}, 5 \mathrm{~m}$, city garden, netting, 8 Mar. 2008, R. Dobosz leg. (USMB); 1 specimen,

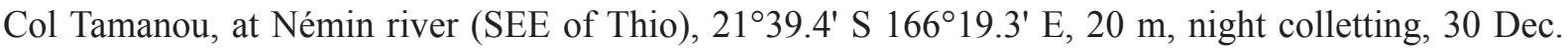
2006, R. Dobosz and M. Wanat leg. (USMB); 2 specimens, Mt Panié, [-20.58860 164.77020], 13001640 m, 28 Jul. 1971, J.L. Gressitt leg. (BPBM); 1 specimen, ditto, [-20.55987 164.78800], 200-500 m, 27 Jul. 1971, J.L. Gressitt leg. (BPBM); 1 specimen, Mt Pouedihi, [-22.16667 166.68333], 560 m, sweeping, 4 Aug. 1971, J. Holloway leg. (BPBM); 1 specimen, Grand Lac, [-22.26832 166.91904], maquis scrub, 9-10 Aug. 1971, J. Holloway leg. (BPBM); 1 specimen, Baie du Prony: W side, [-22.36243 166.80733], 0-10 m, sweeping, 14 Aug. 1979, W.C. Gagné and G.A. Samuelson leg. (BPBM); 1 specimen, Dumbea Valley, [-22.00664 166.50736], Jun. 1950, N.L.H. Krauss leg. (BPBM); 28 specimens, 4 km SW Col de Mouirange, [-22.25605 166.68555], 20 m, 10 Aug. 1979, G.M. Nishida leg. (BPBM); 4 specimens, ditto, on maqui vegetation (BPBM); 10 specimens, ditto, on Baeckea ericoides Brongn. \& Gris, W.C. Gagné leg. (BPBM); 1 specimen, ditto, on Lepidosperma perteres C.B. Clarke (BPBM); 4 specimens, ditto, on Grevillea gillivrayi flowers, G.A. Samuelson leg. (BPBM); 28 specimens, 30 km NW Col de Mouirange [-22.210252 166.689641], 175 m, 10 Aug. 1979, G.M. Nishida leg. (BPBM); 21 specimens, ditto, on Gmelina neocaledonica $\mathrm{S}$. Moore young plants leaves w/mines [= with mines], G.A. Samuelson leg. (BPBM); 1 specimen, Mt Mou, [-22.05937 166.34926], 1200 m, 19 Jan. 1972, J.L. Gressitt leg. (BPBM); 3 specimens, ditto, [-22.06168 166.35080], 9 Mar. 1972 (BPBM); 3 specimens, ditto, [-22.06150 166.35377], 1000 m, on Cunonia, 9 Mar. 1972 (BPBM); 2 specimens, Wara, [-21.26667 165.60000], 100 m, on Grevillea, 6 Apr. 1981, J.L. Gressitt leg. (BPBM); 16 specimens, Bâ - Moneo (Pau Bâ Moneo), [-21.18386 165.51701], 100 m, 1 Aug. 1971, J.L. Gressitt leg. (BPBM); 1 specimen, pass between Bâ and Moneo, [-21.18372 165.51361], 200 m, J.L. Gressitt leg. (BPBM); 1 specimen, ditto, on Metrosideros? (BPBM); 2 specimens, ditto, on Baeckea (BPBM). 


\section{Taxonomic notes}

Arsipoda isola is a light bronze, metallic species clearly distinguishable by the strongly impressed and unevenly sized punctuation of pronotum (Fig. 4D).

Median lobe of aedeagus (Fig. 9A) in ventral view laterally parallel, apically triangular, with a median tooth apically truncate; ventral channel wide, elongate, open in apical part, then partially closed; in lateral view median lobe straight and thickset; dorsal ligula strongly tapered, from apical third to almost
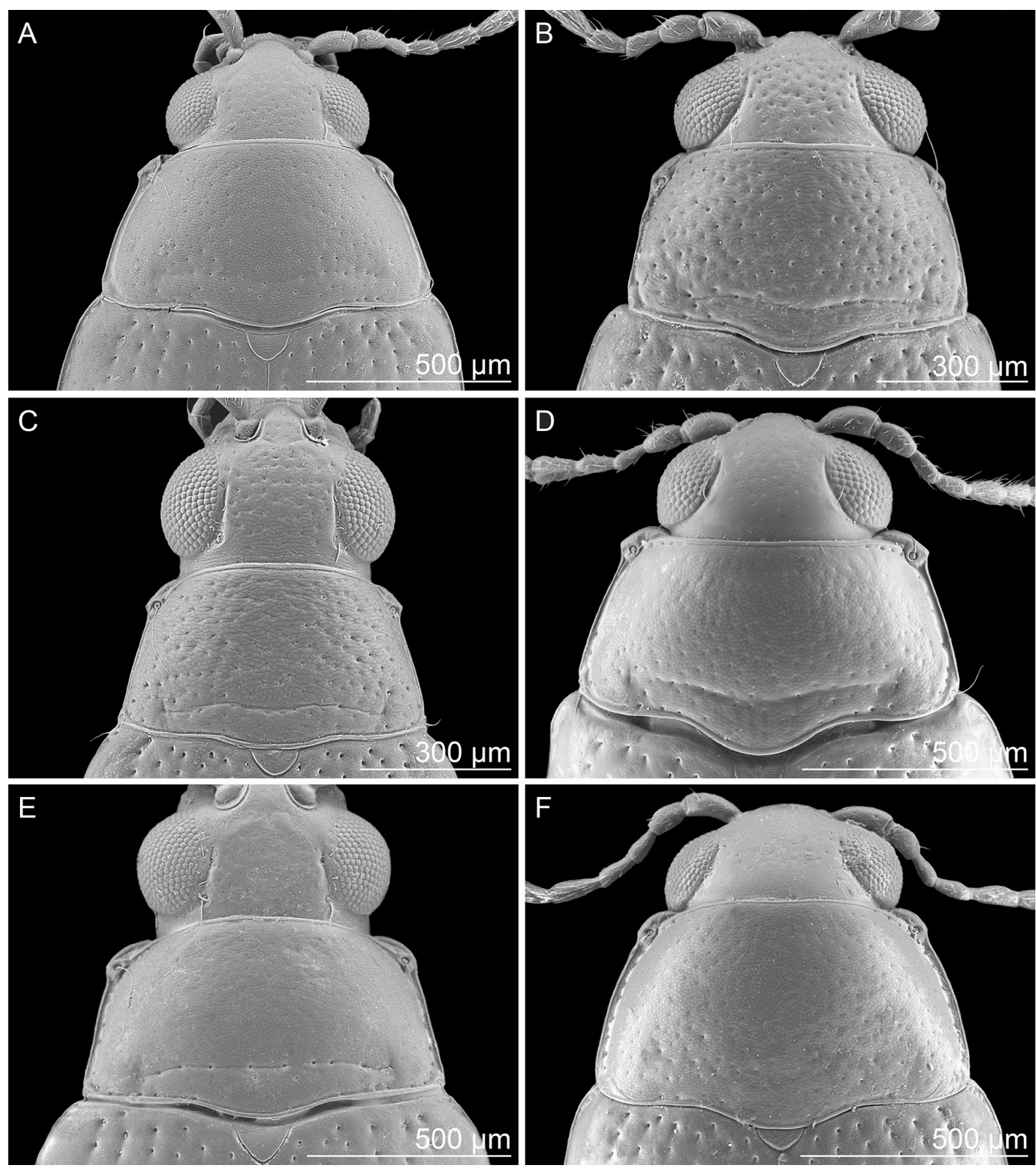

Fig. 5. Head and pronotum. A. Arsipoda povilaensis sp. nov, Pic d'Amoa. B. A. punctata sp. nov., Mueo. C. A. rutai sp. nov., Dzumac Mts. D. A. transversa sp. nov., Mt. Koghi. E. A. wanati sp. nov., Mt Humboldt. F. A. yiambiae Samuelson, 1973, Yiambi. 
the apex of median lobe of aedeagus. Spermatheca (Fig. 11I) with elongate, cylindrical basal part; collum slightly longer than apical part; ductus short, preapically inserted, with proximal part very close to spermathecal capsule.

\section{Biometry}

Male ( $\mathrm{n}=10$; mean and standard deviation; range): $\mathrm{LE}=1.38 \pm 0.07 \mathrm{~mm}(1.23 \leq \mathrm{LE} \leq 1.45 \mathrm{~mm}) ; \mathrm{WE}=$ $0.88 \pm 0.06 \mathrm{~mm}(0.78 \leq \mathrm{WE} \leq 0.95 \mathrm{~mm}) ; \mathrm{LP}=0.42 \pm 0.03 \mathrm{~mm}(0.35 \leq \mathrm{LP} \leq 0.48 \mathrm{~mm}) ; \mathrm{WP}=0.64 \pm$ $0.03 \mathrm{~mm}(0.58 \leq \mathrm{WP} \leq 0.70 \mathrm{~mm}) ; \mathrm{LAN}=1.16 \pm 0.09 \mathrm{~mm}(1.03 \leq \mathrm{LAN} \leq 1.35 \mathrm{~mm}) ; \mathrm{LAED}=0.85 \pm$ $0.03 \mathrm{~mm}(0.81 \leq \mathrm{LAED} \leq 0.90 \mathrm{~mm}) ; \mathrm{LB}=1.98 \pm 0.10 \mathrm{~mm}(1.78 \leq \mathrm{LB} \leq 2.16 \mathrm{~mm}) ; \mathrm{LE} / \mathrm{LP}=3.27 \pm 0.14$ $(3.05 \leq \mathrm{LE} / \mathrm{LP} \leq 3.50) ; \mathrm{WE} / \mathrm{WP}=1.37 \pm 0.04(1.32 \leq \mathrm{WE} / \mathrm{WP} \leq 1.44) ; \mathrm{WP} / \mathrm{LP}=1.52 \pm 0.06(1.47 \leq \mathrm{WP} /$ $\mathrm{LP} \leq 1.64) ; \mathrm{WE} / \mathrm{LE}=0.64 \pm 0.02(0.60 \leq \mathrm{WE} / \mathrm{LE} \leq 0.66) ; \mathrm{LAN} / \mathrm{LB}=0.59 \pm 0.02(0.56 \leq \mathrm{LAN} / \mathrm{LB} \leq$ $0.63) ; \mathrm{LE} / \mathrm{LAED}=1.61 \pm 0.08(1.51 \leq \mathrm{LE} / \mathrm{LAED} \leq 1.76)$.

Female ( $\mathrm{n}=10$; mean and standard deviation; range): $\mathrm{LE}=1.56 \pm 0.08 \mathrm{~mm}(1.43 \leq \mathrm{LE} \leq 1.68 \mathrm{~mm})$; $\mathrm{WE}=1.00 \pm 0.04 \mathrm{~mm}(0.93 \leq \mathrm{WE} \leq 1.05 \mathrm{~mm}) ; \mathrm{LP}=0.46 \pm 0.03 \mathrm{~mm}(0.43 \leq \mathrm{LP} \leq 0.53 \mathrm{~mm}) ; \mathrm{WP}=$ $0.72 \pm 0.03 \mathrm{~mm}(0.66 \leq \mathrm{WP} \leq 0.75 \mathrm{~mm}) ; \mathrm{LAN}=1.19 \pm 0.05 \mathrm{~mm}(1.10 \leq \mathrm{LAN} \leq 1.25 \mathrm{~mm}) ; \mathrm{LSPc}=$ $0.19 \pm 0.01 \mathrm{~mm}(0.18 \leq \mathrm{LSPc} \leq 0.21 \mathrm{~mm}) ; \mathrm{LB}=2.21 \pm 0.09 \mathrm{~mm}(2.16 \leq \mathrm{LB} \leq 2.31 \mathrm{~mm}) ; \mathrm{LE} / \mathrm{LP}=$ $3.38 \pm 0.13(3.19 \leq \mathrm{LE} / \mathrm{LP} \leq 3.71) ; \mathrm{WE} / \mathrm{WP}=1.39 \pm 0.03(1.33 \leq \mathrm{WE} / \mathrm{WP} \leq 1.43) ; \mathrm{WP} / \mathrm{LP}=1.55 \pm 0.09$ $(1.43 \leq \mathrm{WP} / \mathrm{LP} \leq 1.71) ; \mathrm{WE} / \mathrm{LE}=0.64 \pm 0.02(0.61 \leq \mathrm{WE} / \mathrm{LE} \leq 0.67) ; \mathrm{LAN} / \mathrm{LB}=0.54 \pm 0.01(0.52 \leq$ $\mathrm{LAN} / \mathrm{LB} \leq 0.55) ; \mathrm{LE} / \mathrm{LSPc}=8.06 \pm 0.30(7.42 \leq \mathrm{LE} / \mathrm{LSPc} \leq 8.38)$.

\section{Distribution}

Northern, Central and most of Southern Grande Terre (Fig. 14A).

\section{Ecological notes}

Collected in plantation, maquis, dry scrub, forest edge, humid forest, moss forest, subsummit forest, from the coast to over $1350 \mathrm{~m}$ a.s.1. Found on Baeckea ericoides, Tristania sp., Metrosideros sp. (Myrtaceae), Lepidosperma perteres (Cyperaceae) Grevillea gillivrayi, Stenocarpus sp. (Proteaceae), Gmelina neocaledonica (Lamiaceae), Cunonia sp. and Pancheria elliptica Pamp. (Cunoniaceae), Sapium sp. (Euphorbiaceae); Hibbertia spp. (Dilleniaceae). Samuelson (1994) reported A. isola on plants of Phelline (Aquifoliaceae) too. Gómez-Zurita et al. (2010) report molecular evidence associating this species with Ericaceae. Pollen found in the gut of some specimens.

\section{Arsipoda longifrons sp. nov. urn:1sid:zoobank.org:act:0488C97F-BF4D-4467-AAA0-D398BED562BB}

Figs 2A, 9B, 11J, 15A

\section{Diagnosis}

This species is similar in shape, sculpture and often color to A. gomezzuritai sp. nov. and A. rostrata (Figs 2A, F, 3C). They are distinguishable from other Arsipoda species by the elongate antennae (LAN/ LB: $\hat{O} \geq 0.70$, + generally $\geq 0.60$ ) and the elongate frons with narrow frontal carina. The three species can be reliably distinguished by the shape of genitalia (Figs 9B, G, 10D, 11J, M, S).

\section{Etymology}

The name is a Latinized word referring to the elongate frons of the species.

\section{Type material}

\section{Holotype}

NEW CALEDONIA (S): §, Humboldt (S track), -21.90517 166.35621, 800-950 m, maquis, beating along track to Col de Vulcain, 9 Nov. 2008, M. Wanat leg. (MNHN). 

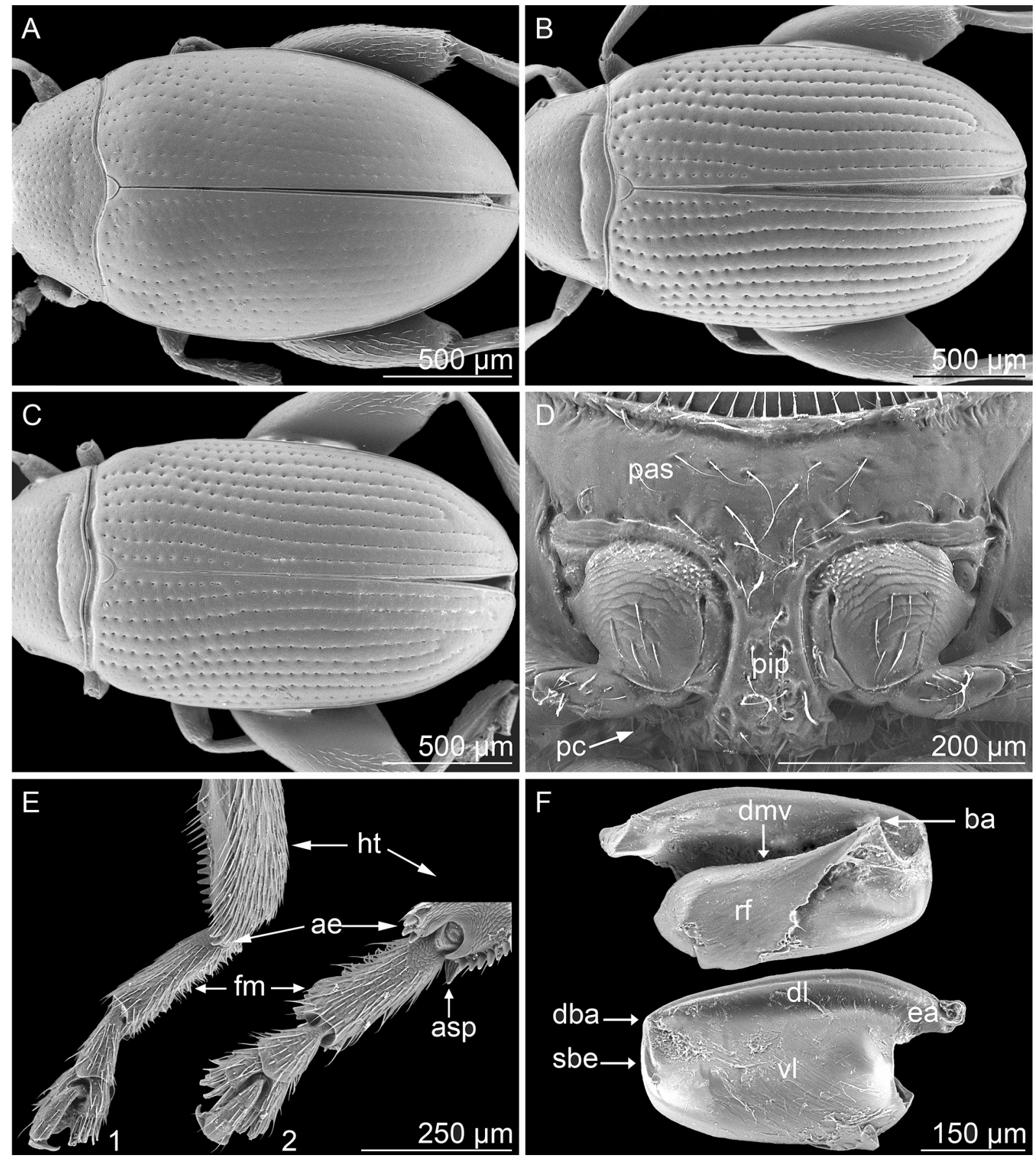

Fig. 6. A-C. Elytra. A. Arsipoda atra sp. nov., Aoupinié. B. A. geographica Gómez-Zurita, 2010, Dzumac Mts (base). C. A. shirleyae Samuelson, 1973, Chute de la Madeleine. D. Prosternum of A. punctata sp. nov., Mueo. E. Hind tibia and tarsus of A. communis sp. nov., Dzumac Mts (1) and A. shirleyae Samuelson, 1973, Pic du Pin (2). F. Metafemoral spring of $A$. communis sp. nov., Dzumac Mts. Abbreviations: ae = apical emargination with thickset spines; asp = apical spur of hind tibia; $\mathrm{ba}=$ basal angle of ventral lobe; $\mathrm{dba}=$ dorsal-basal angle of metafemoral spring; $\mathrm{dl}=$ dorsal lobe; $\mathrm{dmv}=$ dorsal margin of ventral lobe; ea $=$ extended arm of dorsal lobe; $\mathrm{fm}=$ first metatarsomere; $\mathrm{ht}=$ hind tibia; pas = prosternal antecoxal space; $\mathrm{pc}=$ procoxal cavities posteriorly open (arrows); pip = prosternal intercoxal process; $\mathrm{rf}=$ recurve flange; sbe = spring basal edge; $\mathrm{vl}=$ ventral lobe. 


\title{
Paratype
}

NEW CALEDONIA: 1 , same data as the holotype (MNHW).

\section{Description of the holotype $(\stackrel{\jmath}{)})$}

Body moderately elongate and convex (Fig. $2 \mathrm{~A}$ ); $\mathrm{LB}=2.72 \mathrm{~mm}$; maximum pronotal width at base $(\mathrm{WP}=0.89 \mathrm{~mm})$; maximum elytral width near the middle $(\mathrm{WE}=1.30 \mathrm{~mm})$. Dorsum bronze, metallic. Frons and vertex irregularly wrinkled and punctate; vertex concave; head grooves moderately impressed, not continued to postantennal region; genae and frontal carina clearly elongate; antennae clearly longer than $1 / 2$ body length $(\mathrm{LAN}=1.98 \mathrm{~mm} ; \mathrm{LAN} / \mathrm{LB}=0.73$ ), basally light brown, gradually darker toward apex; LA: 100:64:71:86:107:107:118:121:121:111:139. Pronotum slightly trapezoidal, weakly transverse $(\mathrm{LP}=0.58 \mathrm{~mm} ; \mathrm{WP} / \mathrm{LP}=1.54)$, laterally weakly concave; anterior angles not prominent; antebasal transverse sulcus clearly impressed, distinctly sinuate; lateral fovea not clearly distinguishable; pronotal punctuation dense, small and shallow; surface microreticulate. Elytra moderately elongate (LE = $1.98 \mathrm{~mm}$; WE/LE $=0.66$ ), laterally clearly arcuate; punctuation clearly larger than pronotal punctures, clearly impressed; interstriae smooth, micropunctuate. Legs yellowish, with darkened femora; first proand mesotarsomeres distinctly dilated; adhesive setae present on ventral side of first pro-, meso- and, partially, metatarsomeres. Venter brownish; last abdominal ventrite laterally incised, without special preapical impressions. Median lobe of aedeagus (LAED $=1.14 \mathrm{~mm}$; LE/LAED $=1.73$ ) (Fig. 9B) in ventral view thickened, slightly narrower at apical fourth, and clearly narrower at base; apical part subrounded, with a wide median tooth; ventral surface smooth; in lateral view, median lobe curved at basal fourth, then straight; dorsal ligula short, from apical third to preapical part of median lobe of aedeagus, triangular, with wide base.

\section{Biometry}

Male $(\mathrm{n}=1): \mathrm{LE}=1.98 \mathrm{~mm} ; \mathrm{WE}=1.30 \mathrm{~mm} ; \mathrm{LP}=0.58 \mathrm{~mm} ; \mathrm{WP}=0.89 \mathrm{~mm} ; \mathrm{LAN}=1.98 \mathrm{~mm} ; \mathrm{LAED}=$ $1.14 \mathrm{~mm} ; \mathrm{LB}=2.72 \mathrm{~mm} ; \mathrm{LE} / \mathrm{LP}=3.43 ; \mathrm{WE} / \mathrm{WP}=1.46 ; \mathrm{WP} / \mathrm{LP}=1.54 ; \mathrm{WE} / \mathrm{LE}=0.66 ; \mathrm{LAN} / \mathrm{LB}=$ 0.73; $\mathrm{LE} / \mathrm{LAED}=1.73$. Female $(\mathrm{n}=1): \mathrm{LE}=2.13 \mathrm{~mm} ; \mathrm{WE}=1.43 \mathrm{~mm} ; \mathrm{LP}=0.63 \mathrm{~mm} ; \mathrm{WP}=0.93 \mathrm{~mm}$; $\mathrm{LAN}=1.90 \mathrm{~mm} ; \mathrm{LSPc}=0.21 \mathrm{~mm} ; \mathrm{LB}=2.91 \mathrm{~mm} ; \mathrm{LE} / \mathrm{LP}=3.40 ; \mathrm{WE} / \mathrm{WP}=1.54 ; \mathrm{WP} / \mathrm{LP}=1.48 ; \mathrm{WE} /$ $\mathrm{LE}=0.67 ; \mathrm{LAN} / \mathrm{LB}=0.65 ; \mathrm{LE} / \mathrm{LSPc}=10.22$. Paratype (female) very similar in shape, sculpture and color to the holotype; antennae shorter. Spermatheca (Fig. 11J) with globose-elongate basal part; collum thickset, short, slightly longer than apical part; ductus elongate, apically inserted.

\section{Distribution}

Endemic to Mt Humboldt area, Southern Grande Terre (Fig. 15A).

\section{Ecological notes}

Collected in maquis between 800 and $950 \mathrm{~m}$ a.s.1. No information is available about host plants.

\author{
Arsipoda montana sp. nov. \\ urn:1sid:zoobank.org:act:D410BA08-D43B-438A-A48F-800B1F4CDCB8
}

Figs 2B, 4E, 9C, 14B

\section{Diagnosis}

Arsipoda montana sp. nov. is a bronze, strongly metallic species, quite similar to A. communis sp. nov. in sculpture and color. They can be distinguished by the more transverse pronotum and the more deeply impressed pronotal transverse sulcus in A. montana sp. nov. (Fig. 4B, E). Median lobe of aedeagus is also useful to discriminate between the two species (Figs 8D, 9C). 


\section{Etymology}

The specific epithet is a Latinized adjective referring to the high altitude where the species was collected.

\section{Type material}

\section{Holotype}

NEW CALEDONIA (S): đ̊, Mt Humboldt, -21.8793 166.4232, ca 1400 m, montane maquis, 13 Nov. 2010, R. Ruta and M. Wanat leg. (MNHN).

\section{Paratypes}

NEW CALEDONIA: $2 \hat{\jmath} \hat{\partial}, 1 \stackrel{+}{\circ}$, same data of the holotype (MNHW).

\section{Description of the holotype $(\stackrel{\jmath}{)})$}

Body quite thickset, moderately elongate and convex; $\mathrm{LB}=2.72 \mathrm{~mm}$; maximum pronotal width at base ( $\mathrm{WP}=$ $0.93 \mathrm{~mm})$; maximum elytral width at basal third $(\mathrm{WE}=1.28 \mathrm{~mm})$. Dorsum dark bronze, metallic. Frons and vertex microreticulate and densely punctate; head grooves moderately impressed, not continued to postantennal region; genae and frontal carina short; antennae slightly longer than $1 / 2$ body length (LAN $=1.73 \mathrm{~mm}$; LAN/ LB = 0.63), brown, slightly paler basally; LA: 100:64:64:73:91:91:100:100:100:91:127. Pronotum trapezoidal, clearly transverse ( $\mathrm{LP}=0.53 \mathrm{~mm} ; \mathrm{WP} / \mathrm{LP}=1.76$ ), laterally straight (Fig. $4 \mathrm{E})$; anterior angles slightly prominent, obliquely beveled; antebasal transverse sulcus strongly impressed, straight; lateral fovea moderately impressed; pronotal punctuation small and shallow, dense, evenly distributed on the densely microreticulate surface. Elytra moderately elongate $(\mathrm{LE}=2.00 \mathrm{~mm} ; \mathrm{WE} / \mathrm{LE}=0.64)$, laterally moderately arcuate; punctuation quite small but clearly larger than on pronotum, strongly impressed; interstriae microreticulate and micropunctuate. Legs brownish, with darkened femora; first pro- and mesotarsomeres distinctly dilated; adhesive setae present on ventral side of first pro-, meso- and, partially, metatarsomeres. Venter brown; last abdominal ventrite laterally clearly incised, without special preapical impressions. Median lobe of aedeagus (LAED $=1.25 \mathrm{~mm}$; LE/ LAED $=1.59$ ) (Fig. 9C) slender in ventral view, laterally subparallel, slightly narrower at middle and preapical part; apex acute, slightly rounded, with large, apically rounded median tooth; ventral surface with complete, moderately deep ventral sulcus; in lateral view, median lobe curved at basal half, then straight; dorsal ligula long about half of median lobe, fusiform, quite narrow.

\section{Biometry}

Male ( $\mathrm{n}=3$; range): $1.93 \leq \mathrm{LE} \leq 2.00 \mathrm{~mm} ; 1.25 \leq \mathrm{WE} \leq 1.30 \mathrm{~mm} ; 0.51 \leq \mathrm{LP} \leq 0.55 \mathrm{~mm} ; 0.85 \leq \mathrm{WP} \leq$ $0.93 \mathrm{~mm} ; 1.68 \leq \mathrm{LAN} \leq 1.73 \mathrm{~mm} ; 1.22 \leq \mathrm{LAED} \leq 1.25 \mathrm{~mm} ; 2.63 \leq \mathrm{LB} \leq 2.72 \mathrm{~mm} ; 3.61 \leq \mathrm{LE} / \mathrm{LP} \leq 3.81$; $1.38 \leq \mathrm{WE} / \mathrm{WP} \leq 1.47 ; 1.66 \leq \mathrm{WP} / \mathrm{LP} \leq 1.76 ; 0.64 \leq \mathrm{WE} / \mathrm{LE} \leq 0.65 ; 0.62 \leq \mathrm{LAN} / \mathrm{LB} \leq 0.65 ; 1.58 \leq \mathrm{LE} /$ LAED $\leq 1.62$. Paratypes $(\stackrel{)}{)})$ very similar in shape, sculpture and color to the holotype.

Female $(\mathrm{n}=1): \mathrm{LE}=1.86 \mathrm{~mm} ; \mathrm{WE}=1.25 \mathrm{~mm} ; \mathrm{LP}=0.50 \mathrm{~mm} ; \mathrm{WP}=0.85 \mathrm{~mm} ; \mathrm{LAN}=1.45 \mathrm{~mm} ; \mathrm{LB}=$ $2.66 \mathrm{~mm} ; \mathrm{LE} / \mathrm{LP}=3.73 ; \mathrm{WE} / \mathrm{WP}=1.47 ; \mathrm{WP} / \mathrm{LP}=1.70 ; \mathrm{WE} / \mathrm{LE}=0.67 ; \mathrm{LAN} / \mathrm{LB}=0.55$. Spermatheca not detected.

\section{Distribution}

Endemic to Mt Humboldt, Southern Grande Terre (Fig. 14B).

\section{Ecological notes}

Collected in montane maquis at $1400 \mathrm{~m}$ a.s.l. No information is available about host plants. 


\section{Arsipoda paniensis sp. nov. urn:1sid:zoobank.org:act:B101B881-3488-4D66-BC58-4F795DB7EC73}

Figs 2C, 4F, 9D, 11K, 14B

\section{Diagnosis}

Arsipoda paniensis sp. nov. and A. yiambiae are the only species with a laterally clearly convex pronotum, but they are clearly different in color and size: the first is dark green, metallic, and larger; the second is yellow and smaller. Genitalia are easily distinguishable too (Figs 9D, 10C, 11K, R).

\section{Etymology}

The specific name is a Latinized adjective referring to Mt Panié, the only locality where the species has been collected.

\section{Type material}

\section{Holotype}

NEW CALEDONIA: đ̊, Mt Panié, 1450-1638 m, [-20.58860 164.77020], 4 Apr. 1981, on Symplocaceae: Symplocos, 1687 m, J.L. Gressitt leg. (BPBM).

\section{Paratypes}

NEW CALEDONIA: 3 q, , MtPanié, 1450-1638 m, [-20.58860 164.77020], 4 Apr. 1981, on Symplocaceae: Symplocos sp., 1637 m, J.L. Gressitt leg. (BPBM); 2 우, ditto, on Frecynetia sp. (BPBM); 1 ㅅ, 2 우, ditto, 1450-1648 m, on Rapanea sp. (BPBM); 1 Ô, 4 우, ditto, 1450-1738 m, on Scirpus sp. (BPBM); 1 , ditto, 1647 m, 27-29 Jul. 1971, Gahnia sp. (BPBM); 1 o, ditto, smt, 1638 m, 29 Oct. 1978, sifted litter, J.C. Watt leg (NZAC).

\section{Description of the holotype $(ð)$}

Body moderately elongate, moderately convex (Fig. $2 \mathrm{C}$ ); $\mathrm{LB}=2.53 \mathrm{~mm}$; maximum pronotal width at base $(\mathrm{WP}=0.98 \mathrm{~mm})$; maximum elytral width at apical third $(\mathrm{WE}=1.30 \mathrm{~mm})$. Dorsum black, metallic. Frons and vertex microreticulate and sparsely punctate; head grooves distinctly impressed, not continued to postantennal region; frontal carina wide, moderately elongate; antennae slightly longer than $1 / 2$ body length $(\mathrm{LAN}=1.43 \mathrm{~mm} ; \mathrm{LAN} / \mathrm{LB}=0.56)$ yellowish, gradually and slightly darker toward apex; LA:100:65:54:
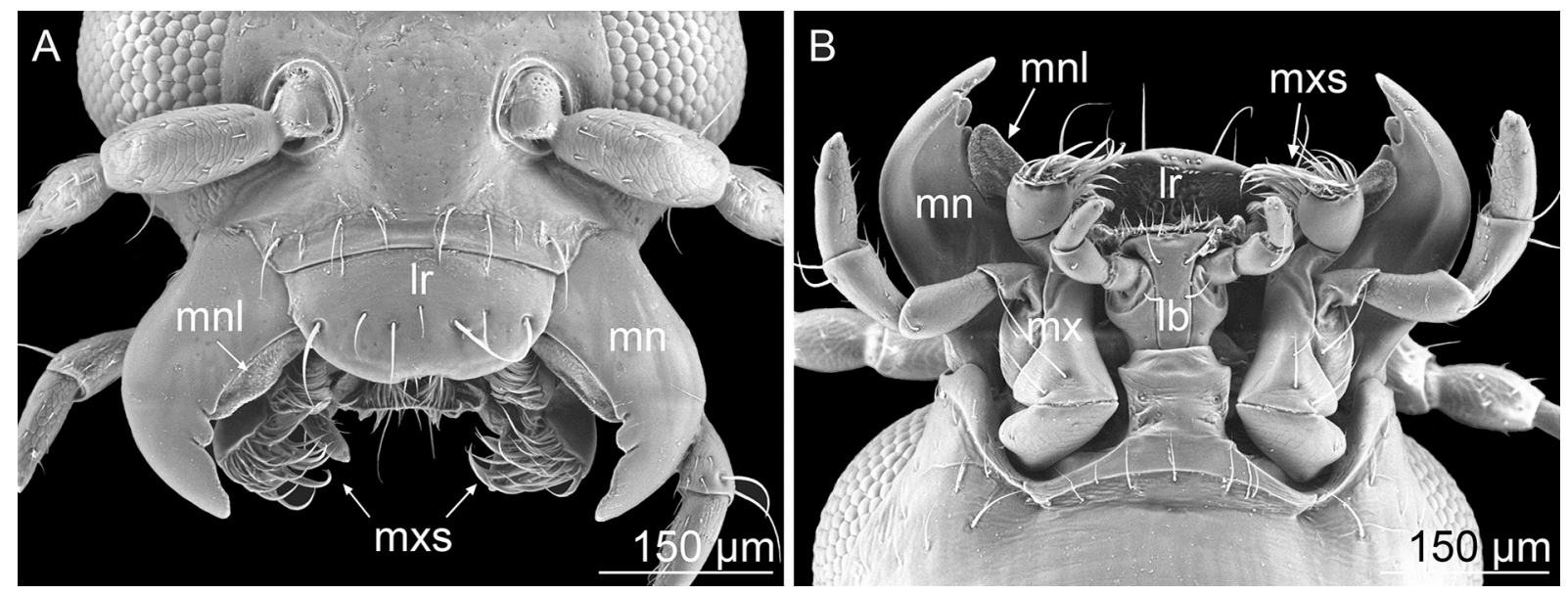

Fig. 7. Mouthparts of A. shirleyae Samuelson, 1973, Mt Koghi. A. Dorsal view. B. Ventral view. Abbreviations: $\mathrm{lb}=$ labium; $\mathrm{lr}=$ labrum; $\mathrm{mn}=$ mandible; $\mathrm{mnl}=$ mandibular lobe; $\mathrm{mx}=$ maxilla; mxs $=$ maxillary setae. 
61:88:77:92:85:88:92:108. Pronotum slightly trapezoidal, distinctly transverse $(\mathrm{LP}=0.60 \mathrm{~mm} ; \mathrm{WP} / \mathrm{LP}=$ 1.63), laterally convex (Fig. 4F); anterior angles weakly prominent; antebasal transverse sulcus weakly impressed, nearly straight, without clearly distinguishable lateral fovea; pronotal surface microreticulate and punctulate, with very small, moderately dense punctures. Elytra moderately elongate (LE $=2.00 \mathrm{~mm}$; $\mathrm{WE} / \mathrm{LE}=0.65$ ), laterally slightly arcuate; punctuation clearly larger than pronotal punctures and clearly impressed; interstriae microreticulate. Legs brownish with paler knees and darker hind femora; first proand mesotarsomeres clearly dilated; adhesive setae present on ventral side of first pro-, meso- and, partially, metatarsomeres. Venter black; last abdominal ventrite laterally incised, with shallow median impression. Median lobe of aedeagus ( $\mathrm{LAED}=1.10 \mathrm{~mm}$; $\mathrm{LE} / \mathrm{LAED}=1.83$ ) (Fig. 9D) in ventral view slightly tapered toward apex; apex triangular-obtuse; ventral sulcus thin and shallow; in lateral view, median lobe slightly and evenly curved; dorsal ligula from half length to apex, narrowed toward apex.

\section{Biometry}

Male $(\mathrm{n}=2): \mathrm{LE}=2.00,2.03 \mathrm{~mm} ; \mathrm{WE}=1.30,1.30 \mathrm{~mm} ; \mathrm{LP}=0.60,0.61 \mathrm{~mm} ; \mathrm{WP}=0.98,0.99 \mathrm{~mm}$; $\mathrm{LAN}=1.43,1.55 \mathrm{~mm} ; \mathrm{LAED}=1.10 \mathrm{~mm}$, und.; $\mathrm{LB}=2.53,2.63 \mathrm{~mm} ; \mathrm{LE} / \mathrm{LP}=3.33,3.31 ; \mathrm{WE} / \mathrm{WP}=$ $1.33,1.32 ; \mathrm{WP} / \mathrm{LP}=1.63,1.61 ; \mathrm{WE} / \mathrm{LE}=0.65,0.64 ; \mathrm{LAN} / \mathrm{LB}=0.56,0.59 ; \mathrm{LE} / \mathrm{LAED}=1.83 \mathrm{~mm}$, und.

Female ( $\mathrm{n}=10$; mean and standard deviation; range): $\mathrm{LE}=2.09 \pm 0.12 \mathrm{~mm}(1.88 \leq \mathrm{LE} \leq 2.23 \mathrm{~mm})$; $\mathrm{WE}=1.34 \pm 0.08 \mathrm{~mm}(1.20 \leq \mathrm{WE} \leq 1.43 \mathrm{~mm}) ; \mathrm{LP}=0.61 \pm 0.04 \mathrm{~mm}(0.54 \leq \mathrm{LP} \leq 0.65 \mathrm{~mm}) ; \mathrm{WP}=$ $1.01 \pm 0.07 \mathrm{~mm}(0.90 \leq \mathrm{WP} \leq 1.10 \mathrm{~mm}) ; \mathrm{LAN}=1.41 \pm 0.07 \mathrm{~mm}(1.30 \leq \mathrm{LAN} \leq 1.50 \mathrm{~mm}) ; \mathrm{LSPc}=$ $0.18 \pm 0.01 \mathrm{~mm}(0.17 \leq \mathrm{LSPc} \leq 0.20 \mathrm{~mm}) ; \mathrm{LB}=2.67 \pm 0.17 \mathrm{~mm}(2.38 \leq \mathrm{LB} \leq 2.84 \mathrm{~mm}) ; \mathrm{LE} / \mathrm{LP}=$ $3.42 \pm 0.08(3.31 \leq \mathrm{LE} / \mathrm{LP} \leq 3.57) ; \mathrm{WE} / \mathrm{WP}=1.32 \pm 0.02(1.30 \leq \mathrm{WE} / \mathrm{WP} \leq 1.34) ; \mathrm{WP} / \mathrm{LP}=1.66 \pm 0.03$ $(1.62 \leq \mathrm{WP} / \mathrm{LP} \leq 1.70) ; \mathrm{WE} / \mathrm{LE}=0.64 \pm 0.01(0.63 \leq \mathrm{WE} / \mathrm{LE} \leq 0.65) ; \mathrm{LAN} / \mathrm{LB}=0.52 \pm 0.02(0.50 \leq$ $\mathrm{LAN} / \mathrm{LB} \leq 0.56) ; \mathrm{LE} / \mathrm{LSPc}=11.38 \pm 0.84(10.05 \leq \mathrm{LE} / \mathrm{LSPc} \leq 12.64)$.

Paratypes very similar in shape, sculpture and color to the holotype. Antennae slightly shorter in female. Spermatheca (Fig. 11K) with pear-shaped basal part, collum about as long as apical part; ductus thickset, elongate, U-shaped, apically inserted.

\section{Distribution}

Endemic to Mt Panié, Northern Grande Terre (Fig. 14B).

\section{Ecological notes}

The collecting localities are located in a dense, evergreen humid forest area between 1450 and $1738 \mathrm{~m}$ a.s.1. Found on Scirpus sp. and Gahnia sp. (Cyperaceae), Frecynetia sp. (Pandanaceae), Rapanea sp. (Primulaceae), Symplocos sp. (Symplocaceae). Pollen found in the gut of some specimens.

\section{Arsipoda povilaensis sp. nov. urn:Isid:zoobank.org:act:6E5BDE56-5D9F-4E95-AC92-7CA05D7CAD8E}

Figs 2D, 5A, 9E, 11L, 15B

\section{Diagnosis}

Arsipoda povilaensis sp. nov. shares a thickset, ovoid body with A. atra sp. nov., A. evax and A. transversa sp. nov. (Figs 1B, F, 2D, I). It can be distinguished by the yellowish integuments (A. evax is black and metallic, $A$. atra sp. nov. is black, and $A$. transversa sp. nov. is reddish); compared to A. transversa sp. nov., with which it can be more easily confused, it also displays a less transverse and less convex pronotum (Fig. 5A, D). Genitalia are also clearly distinguishable (Figs 8C, G, 9E, 10A, 11B, F, L, P). 


\section{Etymology}

The specific name is a Latinized adjective formed after Povila, the type locality where the species has been collected.

\section{Type material}

\section{Holotype}

NEW CALEDONIA (N): đ̊, Pic d'Amoa (Povila), -20.95020 165.29320, 400-450 m, rainforest, 17 Nov. 2010, R. Ruta and M. Wanat leg. (MNHN).

\section{Paratypes}

NEW CALEDONIA: 1 $\hat{\delta}, 3$ 우, same data of the holotype (MNHW); 1 o, Pic d'Amoa (Povila), -20.9510 165.29120, 400 m, lower rainforest, 18 Nov. 2010, M. Wanat and R. Ruta leg. (MNHW); 3 q 9 , ditto, end $0.5 \mathrm{~km}$ of road, -20.95530 165.29100, $450 \mathrm{~m}, 18$ Nov. 2010 (MNHW); 1 , ditto, -20.95659 165.29103, 23 Nov. 2010, M. Wanat leg. (MNHW); 1 \&, Pic d'Amoa, 2057.2' S 165¹7.5' E, $360 \mathrm{~m}$, forest, at light, 14 Jan. 2007, R. Dobosz and M. Wanat leg. (USMB).

\section{Description of the holotype (ð)}

Body thickset, slightly convex (Fig. 2D); LB $=2.38 \mathrm{~mm}$; maximum pronotal width at base $(\mathrm{WP}=0.85 \mathrm{~mm})$; maximum elytral width at basal third $(\mathrm{WE}=1.18 \mathrm{~mm})$. Dorsum yellowish, not metallic. Frons and vertex microreticulate and punctate; head grooves weakly impressed, not continued to postantennal region; genae and frontal carina short; antennae slightly longer than $1 / 2$ body length $(\mathrm{LAN}=1.28 \mathrm{~mm} ; \mathrm{LAN} / \mathrm{LB}=0.54)$, basally yellow, gradually and slightly darker towards apex; LA:100:6 7:56:56:78:78:78:78:89:78:122. Pronotum trapezoidal, clearly transverse $(\mathrm{LP}=0.51 \mathrm{~mm}$; $\mathrm{WP} / \mathrm{LP}=1.66$ ), laterally weakly convex (Fig. 5A); anterior angles distinctly prominent; antebasal transverse sulcus weakly impressed, slightly sinuate, without clearly distinguishable lateral fovea; pronotal surface microreticulate, with small and dense punctuation, similar to that on head. Elytra slightly elongate (LE $=1.73 \mathrm{~mm}$; WE/ $\mathrm{LE}=0.68$ ), laterally clearly arcuate; punctuation quite small, but distinctly larger than on pronotum; interstriae very finely microreticulate and micropunctuate. Legs yellow; first pro- and mesotarsomeres distinctly dilated; adhesive setae present on ventral side of first pro-, meso- and, partially, metatarsomeres. Venter light brown; last abdominal ventrite laterally clearly incised, without special preapical impressions. Median lobe of aedeagus (LAED $=0.94 \mathrm{~mm} ; \mathrm{LE} / \mathrm{LAED}=1.83$ ) (Fig. 9E) in ventral view laterally parallel, slightly narrower in pre-apical part; apex rounded; in lateral view median lobe curved at basal third, then straight; apex sinuate, ventrally oriented; dorsal ligula from half length to apical part of median lobe of aedeagus, narrow.

\section{Biometry}

Male ( $\mathrm{n}=3$; range): $1.60 \leq \mathrm{LE} \leq 1.73 \mathrm{~mm} ; 1.05 \leq \mathrm{WE} \leq 1.08 \mathrm{~mm} ; 0.48 \leq \mathrm{LP} \leq 0.51 \mathrm{~mm} ; 0.81 \leq \mathrm{WP} \leq$ $0.85 \mathrm{~mm} ; 1.25 \leq \mathrm{LAN} \leq 1.28 \mathrm{~mm} ; 0.94 \leq \mathrm{LAED} \leq 0.94 \mathrm{~mm} ; 2.16 \leq \mathrm{LB} \leq 2.38 \mathrm{~mm} ; 3.37 \leq \mathrm{LE} / \mathrm{LP} \leq 3.42$; $1.26 \leq \mathrm{WE} / \mathrm{WP} \leq 1.38 ; 1.66 \leq \mathrm{WP} / \mathrm{LP} \leq 1.79 ; 0.66 \leq \mathrm{WE} / \mathrm{LE} \leq 0.68 ; 0.54 \leq \mathrm{LAN} / \mathrm{LB} \leq 0.59 ; 1.69 \leq \mathrm{LE} /$ $\mathrm{LAED} \leq 1.83$.

Female ( $\mathrm{n}=8$; mean and standard deviation; range): $\mathrm{LE}=1.68 \pm 0.04 \mathrm{~mm}(1.63 \leq \mathrm{LE} \leq 1.74 \mathrm{~mm}) ; \mathrm{WE}=$ $1.10 \pm 0.04 \mathrm{~mm}(1.03 \leq \mathrm{WE} \leq 1.15 \mathrm{~mm}) ; \mathrm{LP}=0.48 \pm 0.01 \mathrm{~mm}(0.48 \leq \mathrm{LP} \leq 0.51 \mathrm{~mm}) ; \mathrm{WP}=0.83 \pm$ $0.03 \mathrm{~mm}(0.80 \leq \mathrm{WP} \leq 0.88 \mathrm{~mm}) ; \mathrm{LAN}=1.13 \pm 0.04 \mathrm{~mm}(1.10 \leq \mathrm{LAN} \leq 1.18 \mathrm{~mm}) ; \mathrm{LSPc}=0.17 \pm$ $0.01 \mathrm{~mm}(0.16 \leq \mathrm{LSPc} \leq 0.18 \mathrm{~mm}) ; \mathrm{LB}=2.24 \pm 0.08 \mathrm{~mm}(2.16 \leq \mathrm{LB} \leq 2.34 \mathrm{~mm}) ; \mathrm{LE} / \mathrm{LP}=3.46 \pm 0.10$ $(3.27 \leq \mathrm{LE} / \mathrm{LP} \leq 3.58) ; \mathrm{WE} / \mathrm{WP}=1.32 \pm 0.04(1.29 \leq \mathrm{WE} / \mathrm{WP} \leq 1.39) ; \mathrm{WP} / \mathrm{LP}=1.72 \pm 0.04(1.66 \leq \mathrm{WP} /$ $\mathrm{LP} \leq 1.76) ; \mathrm{WE} / \mathrm{LE}=0.66 \pm 0.02(0.62 \leq \mathrm{WE} / \mathrm{LE} \leq 0.68) ; \mathrm{LAN} / \mathrm{LB}=0.51 \pm 0.01(0.49 \leq \mathrm{LAN} / \mathrm{LB} \leq$ $0.52) ; \mathrm{LE} / \mathrm{LSPc}=10.06 \pm 0.46(9.38 \leq \mathrm{LE} / \mathrm{LSPc} \leq 10.86)$. 

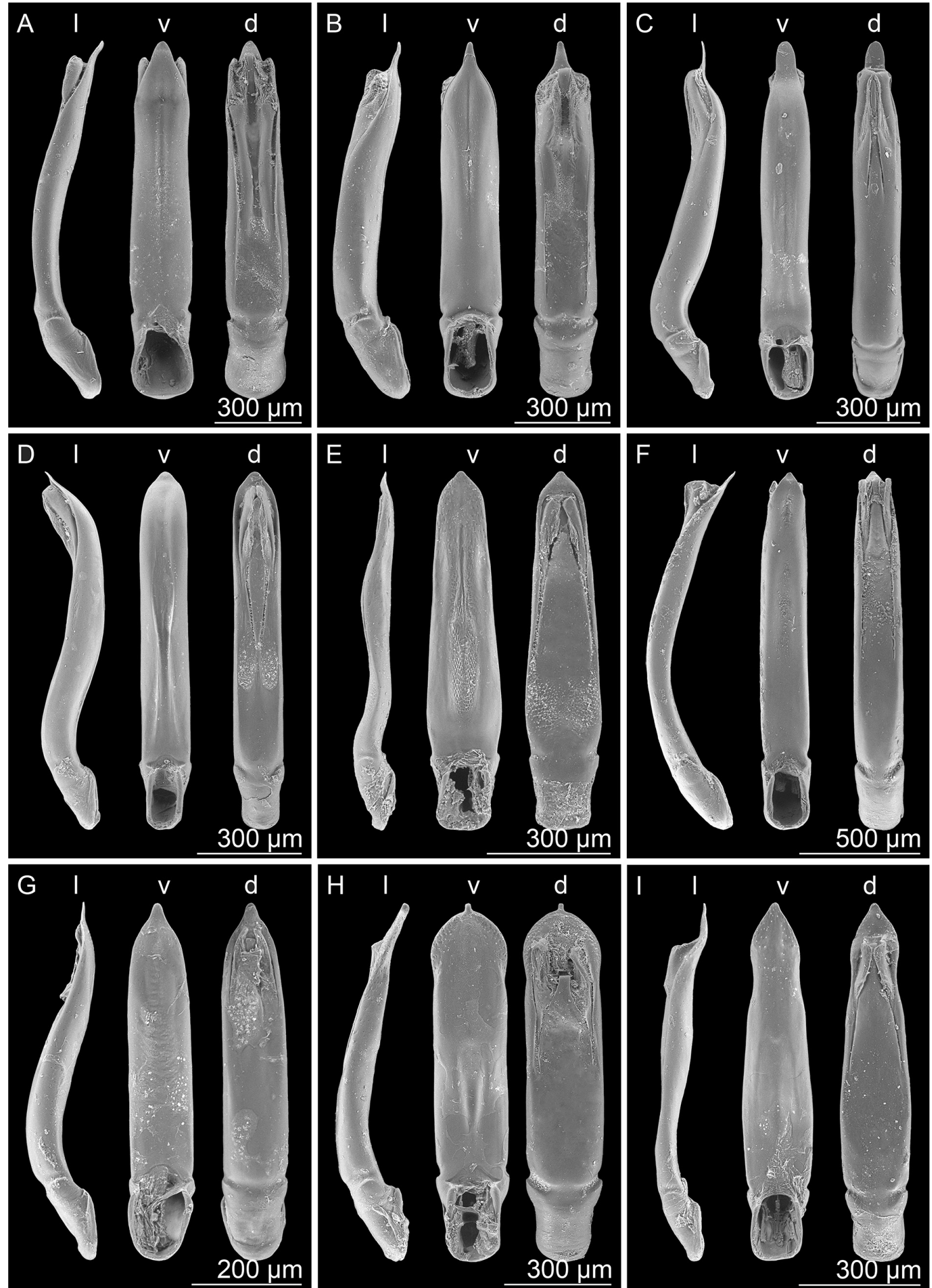

Fig. 8. Median lobe of aedeagus in lateral (l), ventral (v) and dorsal (d) view. A-B. Arsipoda agalma Samuelson, 1973. A. Mt Paniè. B. Col d'Amieu. C. A. atra sp. nov., Aoupinié. D. A. communis sp. nov., Mt Do. E. A. doboszi sp. nov., Haute Rivière Bleue. F. A. elongata sp. nov., Mt Humboldt. G. A. evax Samuelson, 1973, Koghi Mts. H. A. geographica Gómez-Zurita, 2010, Haute Rivière Bleue. I. A. gressitti sp. nov., Farino. 
Paratypes very similar in shape and sculpture to the holotype. Some specimens slightly darker on elytra. Spermatheca (Fig. 11L) with rounded basal part; collum length about twice as long as apical part; ductus thickset, elongate, apically inserted.

\section{Distribution}

Endemic to the Pic d'Amoa, Central Grande Terre (Fig. 15B).

\section{Ecological notes}

Collected in rainforest between 360 and $450 \mathrm{~m}$ a.s.l. No information about host plants is available.

$$
\begin{aligned}
& \text { Arsipoda punctata sp. nov. } \\
& \text { urn:1sid:zoobank.org:act:8176244E-DFAF-4B0E-9639-9F9BA3CB5BD8 }
\end{aligned}
$$

Figs 2E, 5B, 6D, 9F, 15B

\section{Diagnosis}

Arsipoda punctata sp. nov. is an easily distinguishable small, yellow species with a strongly punctate pronotum. Species with similar strong punctuation (A. isola and A. rutai sp. nov.) are very different in color: bronze, metallic in the case of $A$. isola, and very dark with greenish metallic lustre in A. rutai sp. nov.; moreover, the pronotal punctuation is unevenly sized in $A$. isola. Genitalia are also clearly different (Figs 9A, F, H, 11I, N).

\section{Etymology}

The specific epithet is a Latin adjective referring to the strong pronotal punctuation.

\section{Type material}

\section{Holotype}

NEW CALEDONIA: đ̊, Mueo, [-21.310337 165.020997], 20 May 1928, J.D.A. Cockerell leg. (BMNH).

\section{Paratypes}

NEW CALEDONIA: $4 \widehat{\partial} \widehat{\partial}$, same data as the holotype (BMNH).

\section{Description of the holotype $(\circlearrowleft)$}

Body slightly convex (Fig. 2E); $\mathrm{LB}=1.95 \mathrm{~mm}$; maximum pronotal width at base $(\mathrm{WP}=0.74 \mathrm{~mm}$ ); maximum elytral width at basal third $(\mathrm{WE}=0.98 \mathrm{~mm})$. Dorsum yellowish, slightly metallic. Frons and vertex strongly punctate, with microreticulate surface; head grooves short, not continued to postantennal region; genae and frontal carina very short; antennae slightly longer than $1 / 2$ body length (LAN = $1.18 \mathrm{~mm} ; \mathrm{LAN} / \mathrm{LB}=0.61$ ), yellow; LA: 100:56:44:56:78:78:78:78:89:78:100. Pronotum slightly trapezoidal, distinctly transverse ( $\mathrm{LP}=0.45 \mathrm{~mm}$; WP/LP $=1.64)$, laterally straight (Fig. 5B); anterior angles weakly prominent, obliquely beveled; antebasal transverse sulcus moderately impressed, weakly sinuate; lateral fovea almost indistinguishable; pronotal punctuation large, strongly impressed, dense, evenly distributed on the irregularly microreticulate surface. Elytra moderately elongate ( $L E=1.48 \mathrm{~mm}$; $\mathrm{WE} / \mathrm{LE}=0.66$ ), laterally slightly arcuate; punctuation clearly impressed; interstriae microreticulate and micropunctulate.

Legs yellow; first pro- and mesotarsomeres slightly dilated; adhesive setae present on ventral side of first pro-, meso- and, partially, metatarsomeres. Venter brownish; last abdominal ventrite laterally clearly incised, without special pre-apical impressions. Median lobe of aedeagus $($ LAED $=0.74 \mathrm{~mm}$; LE/LAED $=2.01$ ) (Fig. 9F) in ventral view thickset, tapered toward base, apically subtruncate, with median rounded small 
tooth; ventral surface with complete, shallow ventral sulcus, wider at apical 2/3; in lateral view, median lobe slightly curved, gradually thinner toward apex; dorsal ligula elongate, narrow, tapered toward apex.

\section{Biometry}

Male $(\mathrm{n}=5$; mean and standard deviation; range): $\mathrm{LE}=1.52 \pm 0.10 \mathrm{~mm}(1.40 \leq \mathrm{LE} \leq 1.66 \mathrm{~mm})$; $\mathrm{WE}=$ $0.99 \pm 0.06 \mathrm{~mm}(0.90 \leq \mathrm{WE} \leq 1.08 \mathrm{~mm}) ; \mathrm{LP}=0.45 \pm 0.03 \mathrm{~mm}(0.40 \leq \mathrm{LP} \leq 0.48 \mathrm{~mm}) ; \mathrm{WP}=0.75 \pm$ $0.05 \mathrm{~mm}(0.68 \leq \mathrm{WP} \leq 0.82 \mathrm{~mm}) ; \mathrm{LAN}=1.22 \pm 0.07 \mathrm{~mm}(1.12 \leq \mathrm{LAN} \leq 1.30 \mathrm{~mm}) ;$ LAED $=0.77 \pm$ $0.02 \mathrm{~mm}(0.74 \leq \mathrm{LAED} \leq 0.79 \mathrm{~mm}) ; \mathrm{LB}=2.05 \pm 0.19 \mathrm{~mm}(1.85 \leq \mathrm{LB} \leq 2.97 \mathrm{~mm}) ; \mathrm{LE} / \mathrm{LP}=3.58 \pm 0.15$ $(3.38 \leq \mathrm{LE} / \mathrm{LP} \leq 3.83) ; \mathrm{WE} / \mathrm{WP}=1.47 \pm 0.05(1.42 \leq \mathrm{WE} / \mathrm{WP} \leq 2.35) ; \mathrm{WP} / \mathrm{LP}=1.67 \pm 0.03(1.64 \leq$ $\mathrm{WP} / \mathrm{LP} \leq 1.71) ; \mathrm{WE} / \mathrm{LE}=0.65 \pm 0.01(0.64 \leq \mathrm{WE} / \mathrm{LE} \leq 0.66) ; \mathrm{LAN} / \mathrm{LB}=0.60 \pm 0.03(0.54 \leq \mathrm{LAN} /$ $\mathrm{LB} \leq 0.64) ; \mathrm{LE} / \mathrm{LAED}=1.99 \pm 0.08(1.88 \leq \mathrm{LE} / \mathrm{LAED} \leq 2.09)$. Paratypes $(\circlearrowleft)$ very similar in shape, sculpture and color to the holotype.

Female unknown.

\section{Distribution}

Endemic to Mueo area, Central Grande Terre (Fig. 15B).

Arsipoda rostrata Gómez-Zurita, 2010

Figs 2F, 9G, 11M, 14B

Arsipoda rostrata Gómez-Zurita in Gómez-Zurita et al., 2010: 2569.

\section{New material}

NEW CALEDONIA: 4 specimens, Koghi Mts, -22.17809 166.50569, 500-550 m, track to Cascade, forest, 4 Dec. 2008, M. Wanat leg. (MNHW); 1 specimen, ditto, 22 $2^{\circ} 11^{\prime} \mathrm{S} 166^{\circ} 30^{\prime} \mathrm{E}, 500-550 \mathrm{~m}$, humid forest, 21 Jan. 2004 (MNHW); 1 specimen, Dzumac Road (Mt Ouin to Mts Couvélé road junctions), $22^{\circ} 04.1^{\prime} \mathrm{S} 166^{\circ} 26.8^{\prime} \mathrm{E}, 800-900 \mathrm{~m}, 29$ Dec. 2006, M. Wanat leg. (MNHW); 1 specimen, Mt Dzumac (base), km 1.5-3 E Ouin rd jct [= road junction], -22.03710 166.49570, $800 \mathrm{~m}$, rainforest, 6 Dec. 2010, R. Ruta and M. Wanat leg. (MNHW); 1 specimen, ditto (Mt Ouin road junction), $22^{\circ} 01.9^{\prime} \mathrm{S} 166^{\circ} 28.0^{\prime} \mathrm{E}$, $900 \mathrm{~m}$, beating, 28 Dec. 2006, R. Dobosz and M. Wanat leg. (USMB); 1 specimen, Mt Ouin Rd, km 0-0.5 N of Dzumac jct, -22.03180 166.46740, 900 m, 4 Dec. 2010, R. Ruta and M. Wanat leg. (MNHW).

\section{Taxonomic notes}

This species is similar in shape, sculpture and generally color to $A$. gomezzuritai sp. nov. and $A$. longifrons sp. nov. (Figs 2A, F, 3C). They are distinguishable from other Arsipoda species by the elongate antennae (LAN/LB: $\delta \geq 0.70, q$ generally $\geq 0.60$ ) and the elongate frons with narrow frontal carina. The three species can be reliably distinguished by the shape of their genitalia (Figs 9B, G, 10D, 11J, M, S).

Median lobe of aedeagus (Fig. 9G) in ventral view fusiform, with small median tooth; ventral surface with shallow median groove; in lateral view median lobe slightly and regularly curved; dorsal ligula from half length to apical part of median lobe of aedeagus, tapered toward apex. Spermatheca (Fig. 11M) with widely rounded basal part; collum moderately elongate, about twice as long as apical part; ductus quite short, apically inserted.

\section{Biometry}

Male ( $\mathrm{n}=10$; mean and standard deviation; range): $\mathrm{LE}=1.38 \pm 0.07 \mathrm{~mm}(1.23 \leq \mathrm{LE} \leq 1.45 \mathrm{~mm})$; $\mathrm{WE}=$ $0.88 \pm 0.06 \mathrm{~mm}(1.78 \leq \mathrm{WE} \leq 1.95 \mathrm{~mm}) ; \mathrm{LP}=0.42 \pm 0.03 \mathrm{~mm}(0.35 \leq \mathrm{LP} \leq 0.48 \mathrm{~mm}) ; \mathrm{WP}=0.64 \pm$ $0.03 \mathrm{~mm}(0.58 \leq \mathrm{WP} \leq 0.70 \mathrm{~mm}) ; \mathrm{LAN}=1.16 \pm 0.09 \mathrm{~mm}(1.08 \leq \mathrm{LAN} \leq 1.35 \mathrm{~mm}) ; \mathrm{LAED}=0.85 \pm$ 

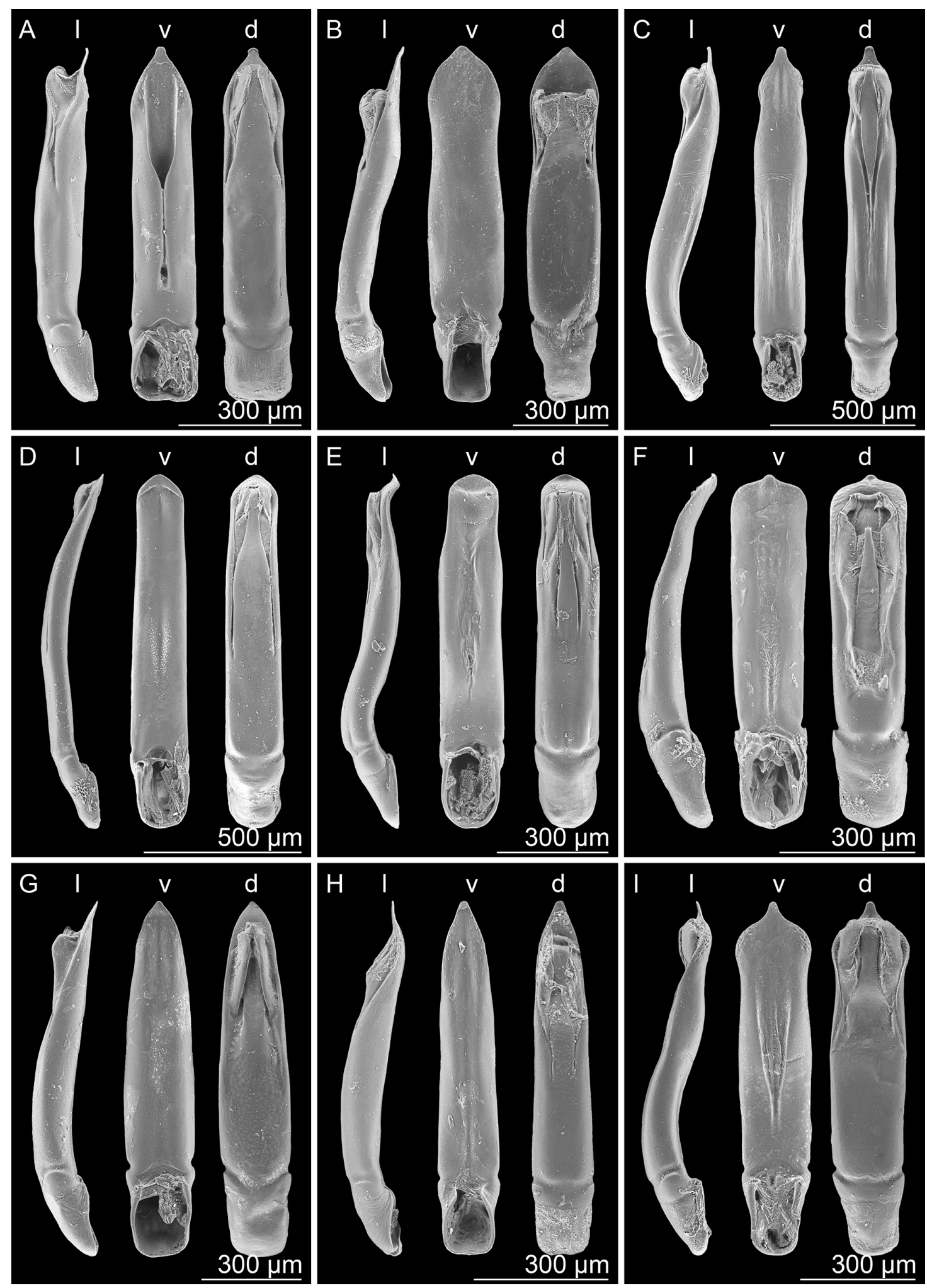

Fig. 9. Median lobe of aedeagus in lateral (1), ventral (v) and dorsal(d) view. A. Arsipoda isola Samuelson, 1973, Bois du Sud. B. A. longifrons sp. nov., Humboldt S track. C. A. montana sp. nov., Mt Humboldt. D. A. paniensis sp. nov., Mt Panié. E. A. povilaensis sp. nov., Pic d'Amoa, Povila. F. A. punctata sp. nov., Mueo. G. A. rostrata Gómez-Zurita, 2010, Koghi Mts. H. A. rutai sp. nov. Poro Plateau. I. A. shirleyae Samuelson, 1973, S Humboldt. 
$0.03 \mathrm{~mm}(0.81 \leq \mathrm{LAED} \leq 0.90 \mathrm{~mm}) ; \mathrm{LB}=1.98 \pm 0.10 \mathrm{~mm}(1.94 \leq \mathrm{LB} \leq 2.16 \mathrm{~mm}) ; \mathrm{LE} / \mathrm{LP}=3.27 \pm 0.14$ $(3.05 \leq \mathrm{LE} / \mathrm{LP} \leq 3.50) ; \mathrm{WE} / \mathrm{WP}=1.37 \pm 0.04(1.32 \leq \mathrm{WE} / \mathrm{WP} \leq 1.44) ; \mathrm{WP} / \mathrm{LP}=1.52 \pm 0.06(1.47 \leq \mathrm{WP} /$ $\mathrm{LP} \leq 1.64) ; \mathrm{WE} / \mathrm{LE}=0.64 \pm 0.02(0.60 \leq \mathrm{WE} / \mathrm{LE} \leq 0.66) ; \mathrm{LAN} / \mathrm{LB}=0.59 \pm 0.02(0.55 \leq \mathrm{LAN} / \mathrm{LB} \leq$ $0.63) ; \mathrm{LE} / \mathrm{LAED}=1.61 \pm 0.08(1.51 \leq \mathrm{LE} / \mathrm{LAED} \leq 1.76)$.

Female ( $\mathrm{n}=10$; mean and standard deviation; range): $\mathrm{LE}=1.56 \pm 0.08 \mathrm{~mm}(1.43 \leq \mathrm{LE} \leq 1.68 \mathrm{~mm})$; $\mathrm{WE}=1.00 \pm 0.04 \mathrm{~mm}(0.93 \leq \mathrm{WE} \leq 1.05 \mathrm{~mm}) ; \mathrm{LP}=0.46 \pm 0.03 \mathrm{~mm}(0.43 \leq \mathrm{LP} \leq 0.53 \mathrm{~mm}) ; \mathrm{WP}=$ $0.72 \pm 0.03 \mathrm{~mm}(0.66 \leq \mathrm{WP} \leq 0.75 \mathrm{~mm}) ; \mathrm{LAN}=1.19 \pm 0.05 \mathrm{~mm}(1.10 \leq \mathrm{LAN} \leq 1.25 \mathrm{~mm}) ; \mathrm{LSPc}=$ $0.19 \pm 0.01 \mathrm{~mm}(0.18 \leq \mathrm{LSPc} \leq 0.21 \mathrm{~mm}) ; \mathrm{LB}=2.21 \pm 0.09 \mathrm{~mm}(2.00 \leq \mathrm{LB} \leq 2.31 \mathrm{~mm}) ; \mathrm{LE} / \mathrm{LP}=$ $3.38 \pm 0.13(3.19 \leq \mathrm{LE} / \mathrm{LP} \leq 3.71) ; \mathrm{WE} / \mathrm{WP}=1.39 \pm 0.03(1.37 \leq \mathrm{WE} / \mathrm{WP} \leq 1.43) ; \mathrm{WP} / \mathrm{LP}=1.55 \pm 0.09$ $(1.43 \leq \mathrm{WP} / \mathrm{LP} \leq 1.71) ; \mathrm{WE} / \mathrm{LE}=0.64 \pm 0.02(0.61 \leq \mathrm{WE} / \mathrm{LE} \leq 0.67) ; \mathrm{LAN} / \mathrm{LB}=0.54 \pm 0.01(0.52 \leq$ $\mathrm{LAN} / \mathrm{LB} \leq 0.55) ; \mathrm{LE} / \mathrm{LSPc}=8.06 \pm 0.30(7.42 \leq \mathrm{LE} / \mathrm{LSPc} \leq 8.38)$.

\section{Distribution}

Southern Grande Terre (Fig. 14B).

\section{Ecological notes}

Collected in rainforest and humid forest between 500 and $900 \mathrm{~m}$ a.s.l. No information is available about host plants. Pollen found in the gut of some specimens.

\section{Arsipoda rutai sp. nov. urn:1sid:zoobank.org:act:E0C9977F-07C0-44C7-9CF0-1CAE88FCB6A4} Figs $2 \mathrm{G}, 5 \mathrm{C}, 9 \mathrm{H}, 11 \mathrm{~N}, 14 \mathrm{~A}$

\section{Diagnosis}

Arsipoda rutai sp. nov. can be easily identified by the very dark color with greenish metallic luster, and rather large, strongly impressed pronotal punctuation. Species with similar strong punctuation $(A$. isola and $A$. punctata sp. nov.) are of a different color: light brown with evident bronze luster (A. isola), and yellowish, slightly metallic (A. punctata sp. nov.); moreover, the pronotal punctuation is unevenly sized in A. isola (Fig. 4D). Genitalia are also clearly different (Figs 9A, F, H, 11I, N).

\section{Etymology}

Male patronym. Named after Dr. Rafal Ruta (University of Wroclaw, Poland), one of its collectors.

\section{Type material}

\section{Holotype}

NEW CALEDONIA(N): ^̊, Poro (6 km SSE), -21.34795 165.69385, $620 \mathrm{~m}$, plateau above mine, forest, 30 Nov. 2008, M. Wanat leg. (MNHN).

\section{Paratypes}

NEW CALEDONIA: 1 , same data as the holotype (MNHW); 1 , Poro Plateau, -21.3483 165.6932, 620 m, forest, night, 28 Nov. 2010, M. Wanat and R. Ruta leg. (MNHW); 1 §, Humboldt (S track), -21.90517 166.35621, 800-950 m, maquis, beating along track to Col du Vulcain, 9 Nov. 2008, M. Wanat

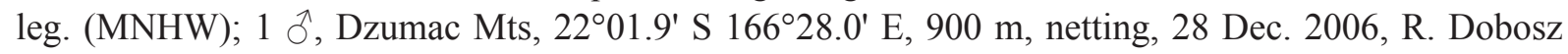

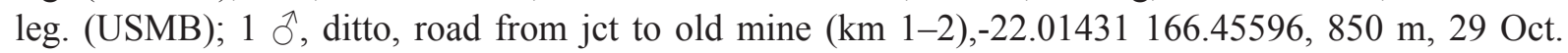
2008, M. Wanat leg. (MNHW); 2 ô $^{\wedge}, 3$ 우, ditto (Mt Ouin road junction), $-22^{\circ} 01.9^{\prime} \mathrm{S} 166^{\circ} 28.0^{\prime} \mathrm{E}$, 900 m, 28 Dec. 2006, M. Wanat and R. Dobosz leg. (MNHW); 1 ð, 2 우, Dzumac Road, S of Mts Couvélé rd jct [= road junction], -22.06913 166.44666, 870-670 m, beating, 31 Oct. 2008, M. Wanat leg.

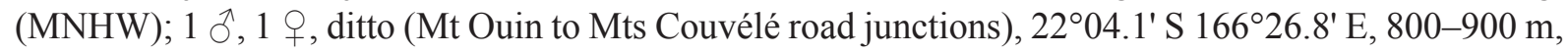


29 Dec. 2006, M. Wanat leg. (MNHW); 1 गे, ditto, km 0-1 E of Ouin rd jet [= road junction], -22.03340 166.47480, 800-900 m, to refuge, maquis, 6 Dec. 2010, M. Wanat and R. Ruta leg. (MNHW); 1 ð, 1 क, Mt Mou, [-22.05937 166.34926], 1220 m, 3 Feb. 1963, J. L. Gressitt leg. (BPBM); 1 क, ditto, [-22.06150 166.35377], 1000-1200 m, 9 Mar. 1972 (BPBM); 1 क, ditto, [-22.06958 166.34952], 500800 m, 2 Nov. 1978, J.S. Dugdale leg. (NZAC); 1 đે, Mt Koghis, [-22.17236 166.51129], 600 m, 12 Oct. 1978, G. Kuschel leg. (NZAC).

\section{Description of the holotype $(ð)$}

Body moderately elongate and convex (Fig. $2 \mathrm{G}$ ); $\mathrm{LB}=1.97 \mathrm{~mm}$; maximum pronotal width at base $(\mathrm{WP}=0.63 \mathrm{~mm})$; maximum elytral width at basal third $(\mathrm{WE}=0.88 \mathrm{~mm})$. Dorsum black, with green metallic reflection. Frons and vertex microreticulate and clearly punctate; head grooves moderately impressed, not continued to postantennal region; genae and frontal carina short; antennae distinctly
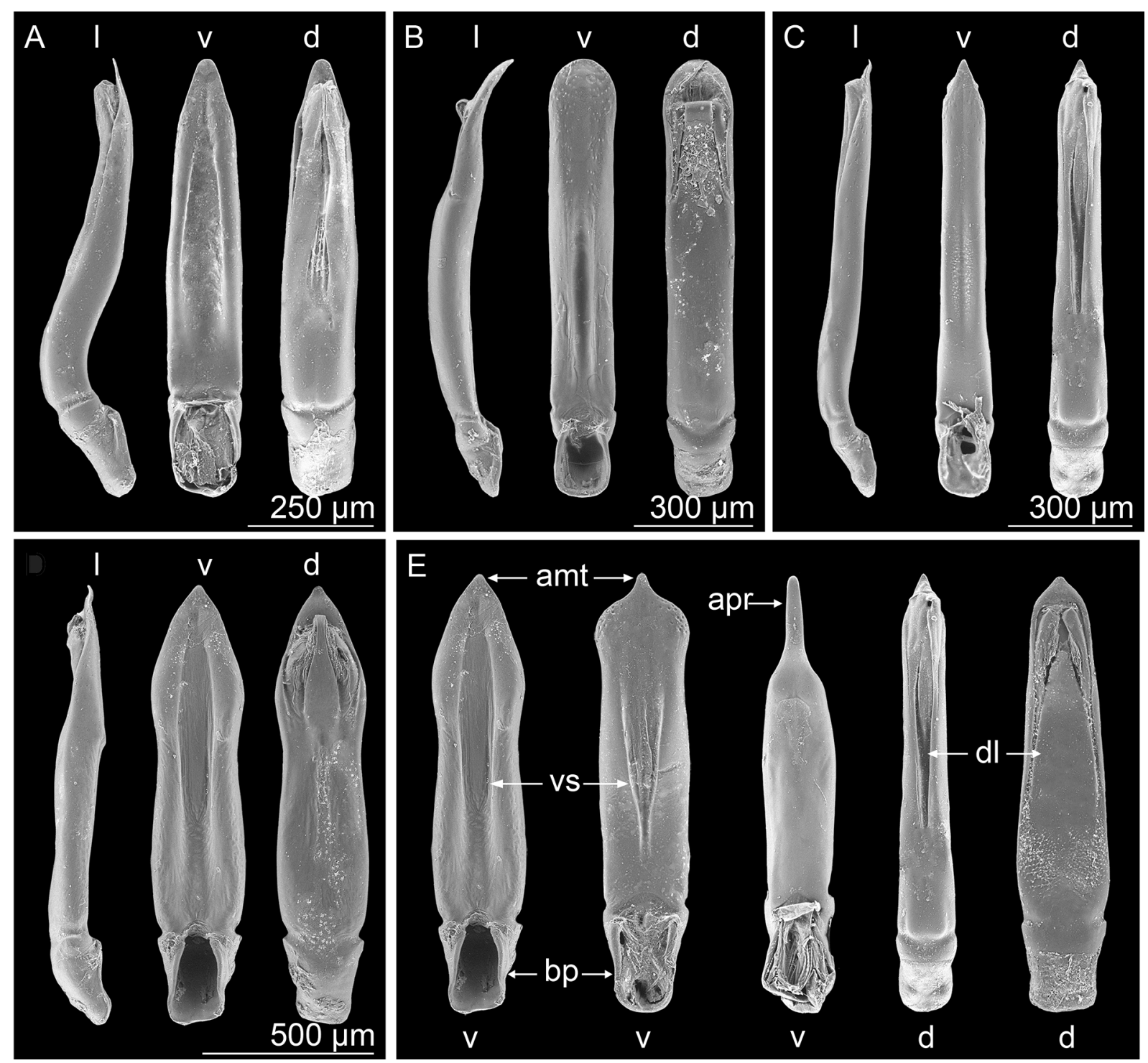

Fig. 10. Median lobe of aedeagus in lateral (l), ventral (v) and dorsal (d) view. A. Arsipoda transversa sp. nov., Mt Koghi. B. A. wanati sp. nov., Mt Humboldt. C. A. yiambiae Samuelson, 1973, Yiambi. D. A. gomezzuritai sp. nov., Dzumac Mts. E. Nomenclature. Abbreviations: amt = apical median tooth; $\mathrm{apr}=$ apical process; $\mathrm{bp}=$ basal part; $\mathrm{dl}=$ dorsal ligula; $\mathrm{vs}=$ ventral sulcus. 
longer than $1 / 2$ body length $(\mathrm{LAN}=1.33 \mathrm{~mm}$; $\mathrm{LAN} / \mathrm{LB}=0.67)$, basally brown, gradually darker towards apex; LA: 100:70:60:95:100:100:100:110:100:95:140. Pronotum trapezoidal, slightly transverse (LP = $0.43 \mathrm{~mm}$; WP/LP = 1.47), laterally straight (Fig. 5C); anterior angles weakly prominent; antebasal transverse sulcus moderately impressed, slightly sinuate, without clearly distinguishable lateral fovea; pronotal surface microreticulate, with dense punctures differently sized. Elytra moderately elongate $(\mathrm{LE}=1.35 \mathrm{~mm} ; \mathrm{WE} / \mathrm{LE}=0.65)$, laterally distinctly arcuate; punctuation large and strongly impressed; interstriae microreticulate and clearly micropunctuate. Legs yellowish with black femora; first pro- and mesotarsomeres weakly dilated; adhesive setae present on ventral side of first pro-, meso- and, partially, metatarsomeres. Venter brown; last abdominal ventrite laterally briefly incised, with clear median dimple. Median lobe of aedeagus $(\mathrm{LAED}=0.79 \mathrm{~mm} ; \mathrm{LE} / \mathrm{LAED}=1.70)($ Fig. $9 \mathrm{H})$ in ventral view slightly tapered toward apex; apical part triangular, with rounded apex; ventral groove narrow, with thin median carina; in lateral view, median lobe slightly curved, especially on dorsum, with straight apex; dorsal ligula from half length to preapical part of median lobe of aedeagus, moderately wide, laterally parallel, apically slightly narrower.

\section{Biometry}

Male ( $\mathrm{n}=10$; mean and standard deviation; range): $\mathrm{LE}=1.44 \pm 0.08 \mathrm{~mm}(1.35 \leq \mathrm{LE} \leq 1.58 \mathrm{~mm})$; $\mathrm{WE}=$ $0.93 \pm 0.06 \mathrm{~mm}(0.85 \leq \mathrm{WE} \leq 1.03 \mathrm{~mm}) ; \mathrm{LP}=0.42 \pm 0.03 \mathrm{~mm}(0.40 \leq \mathrm{LP} \leq 0.48 \mathrm{~mm}) ; \mathrm{WP}=0.66 \pm$ $0.05 \mathrm{~mm}(0.60 \leq \mathrm{WP} \leq 0.74 \mathrm{~mm}) ; \mathrm{LAN}=1.40 \pm 0.09 \mathrm{~mm}(1.30 \leq \mathrm{LAN} \leq 1.58 \mathrm{~mm}) ; \mathrm{LAED}=0.83 \pm$ $0.03 \mathrm{~mm}(0.79 \leq \mathrm{LAED} \leq 0.89 \mathrm{~mm}) ; \mathrm{LB}=2.06 \pm 0.13 \mathrm{~mm}(1.94 \leq \mathrm{LB} \leq 2.28 \mathrm{~mm}) ; \mathrm{LE} / \mathrm{LP}=3.41 \pm 0.13$ $(3.18 \leq \mathrm{LE} / \mathrm{LP} \leq 3.60) ; \mathrm{WE} / \mathrm{WP}=1.41 \pm 0.04(1.38 \leq \mathrm{WE} / \mathrm{WP} \leq 1.50) ; \mathrm{WP} / \mathrm{LP}=1.55 \pm 0.05(1.47 \leq \mathrm{WP} /$ $\mathrm{LP} \leq 1.63) ; \mathrm{WE} / \mathrm{LE}=0.64 \pm 0.01(0.63 \leq \mathrm{WE} / \mathrm{LE} \leq 0.66) ; \mathrm{LAN} / \mathrm{LB}=0.68 \pm 0.01(0.66 \leq \mathrm{LAN} / \mathrm{LB} \leq$ $0.70) ; \mathrm{LE} / \mathrm{LAED}=1.74 \pm 0.05(1.67 \leq \mathrm{LE} / \mathrm{LAED} \leq 1.81)$.

Female ( $\mathrm{n}=8$; mean and standard deviation; range): $\mathrm{LE}=1.58 \pm 0.08 \mathrm{~mm}(1.50 \leq \mathrm{LE} \leq 1.75 \mathrm{~mm}) ; \mathrm{WE}=$ $1.01 \pm 0.06 \mathrm{~mm}(0.95 \leq \mathrm{WE} \leq 1.08 \mathrm{~mm}) ; \mathrm{LP}=0.44 \pm 0.02 \mathrm{~mm}(0.40 \leq \mathrm{LP} \leq 0.45 \mathrm{~mm}) ; \mathrm{WP}=0.70 \pm$ $0.05 \mathrm{~mm}(0.64 \leq \mathrm{WP} \leq 0.79 \mathrm{~mm}) ; \mathrm{LAN}=1.26 \pm 0.04 \mathrm{~mm}(1.18 \leq \mathrm{LAN} \leq 1.33 \mathrm{~mm}) ; \mathrm{LSPc}=0.14 \pm$ $0.01 \mathrm{~mm}(0.12 \leq \mathrm{LSPc} \leq 0.15 \mathrm{~mm}) ; \mathrm{LB}=2.23 \pm 0.12 \mathrm{~mm}(2.13 \leq \mathrm{LB} \leq 2.50 \mathrm{~mm}) ; \mathrm{LE} / \mathrm{LP}=3.63 \pm 0.11$ $(3.39 \leq \mathrm{LE} / \mathrm{LP} \leq 3.75) ; \mathrm{WE} / \mathrm{WP}=1.45 \pm 0.02(1.43 \leq \mathrm{WE} / \mathrm{WP} \leq 1.49) ; \mathrm{WP} / \mathrm{LP}=1.59 \pm 0.06(1.50 \leq \mathrm{WP} /$ $\mathrm{LP} \leq 1.66) ; \mathrm{WE} / \mathrm{LE}=0.64 \pm 0.01(0.63 \leq \mathrm{WE} / \mathrm{LE} \leq 0.66) ; \mathrm{LAN} / \mathrm{LB}=0.57 \pm 0.02(0.52 \leq \mathrm{LAN} / \mathrm{LB} \leq$ $0.60) ; \mathrm{LE} / \mathrm{LSPc}=11.32 \pm 0.78(10.30 \leq \mathrm{LE} / \mathrm{LSPc} \leq 12.50)$.

Paratypes very similar in shape, sculpture and color similar to the holotype. Antennae shorter in females. Spermatheca (Fig. 11N) with globose basal part; apical part short; collum about twice as long as apical part; appendix very elongate; ductus moderately elongate, preapically inserted on dorsal side, with proximal part running distant to spermathecal capsule.

\section{Distribution}

Central and mostly Southern Grande Terre (Fig. 14A).

\section{Ecological notes}

Collected in forest and maquis between 600 and $1200 \mathrm{~m}$ a.s.1. No information is available about host plants. Pollen found in the gut of some specimens.

Arsipoda shirleyae Samuelson, 1973

Figs $2 \mathrm{H}, 6 \mathrm{C}, \mathrm{E}(2), 7 \mathrm{~A}-\mathrm{B}, 9 \mathrm{I}, 11 \mathrm{O}, 15 \mathrm{~A}$

Arsipoda shirleyae Samuelson, 1973.

Arsipoda shirleyae - Samuelson 1989: 409. — Gómez-Zurita et al. 2010: 2560. 


\section{Type material examined}

Holotype

NEW CALEDONIA: §, La Coulee-Yate Road, [-22,21733 166,66790], Jun. 1950, N.L.H. Krauss (BPBM).

\section{Paratypes}

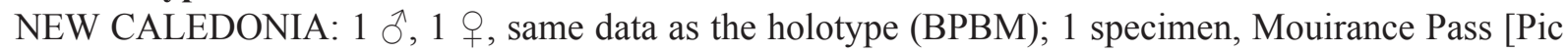
Mouirange], [-22,21667 166,65000], 10 Feb. 1963, N.L.H. Krauss leg. (BMNH).

\section{New material}

NEW CALEDONIA: 1 specimen, Humboldt (S track), -21.90517 166.35621, 800-950 m, maquis, beating down Col du Vulcain, 11 Nov. 2008, M. Wanat leg. (MNHW); 3 specimens, ditto, maquis, beating along track to Col du Vulcain, 9 Nov. 2008 (MNHW); 1 specimen, ditto, 580-800 m, rainforest, beating along track, 11 Nov. 2008 (MNHW); 2 specimens, ditto, 9 Nov. 2008 (MNHW); 3 specimens, ditto, Col du Vulcain (refuge), -21.90319 166.38305, 980 m, night beating (MNHW); 1 specimen, ditto, Botanical Reserve, -21.88267 166.39968, 1130-1280 m, moss forest, 10 Nov. 2008 (MNHW); 3 specimens, S Humboldt trail to Col du Vulcain, -21.90430 166.38100, 800-950 m, maquis, 11 Nov. 2010, M. Wanat and R. Ruta leg. (MNHW); 4 specimens, Plaine du champ de Bataille, $22^{\circ} 18.1^{\prime} \mathrm{S} 166^{\circ} 44.7^{\prime} \mathrm{E}, 40 \mathrm{~m}$, maquis, 17 Dec. 2006, M. Wanat, R. Dobosz and P. Krzyzynski leg. (MNHW); 1 specimen, Pic du Pin [-22.24843 166.82883], beating, 6 Oct. 1978, J.C. Watt leg. (NZAC); 8 specimens, ditto (base), $280 \mathrm{~m}$, plantation, 22 Oct. 2008, M. Wanat leg. (MNHW); 1 specimen, ditto, 23 Oct. 2008 (MNHW); 1 specimen, ditto, at light, 22 Oct. 2008 (MNHW); 2 specimens, ditto, forest (MNHW); 9 specimens, ditto, 23 Oct. 2008 (MNHW); 1 specimen, ditto, beating, forest edge, 2 Dec. 2008 (MNHW); 9 specimens, ditto, -22.24810 166.82920, 280 m, plantation, 31 Oct. 2010, M. Wanat and R. Ruta leg. (MNHW); 1 specimen, ditto, -22.24820 166.82850, $280 \mathrm{~m}$, forest (MNHW); 1 specimen, ditto, -22.24810 166.82880, $280 \mathrm{~m}$, at light (MNHW); 1 specimen, ditto, 22 ${ }^{\circ} 14.9^{\prime} \mathrm{S} 166^{\circ} 49.7^{\prime} \mathrm{E}, 280 \mathrm{~m}$, forest and plantation, 26 Dec. 2006, M. Wanat and R. Dobosz leg. (MNHW); 1 specimen, ditto, 25 Dec. 2006 (MNHW); 1 specimen, ditto, night coll. (lamp and beating) (USMB); 3 specimens, Haute Rivière Bleue, track La Tranchée-Hte Pourina, 22 $04.0^{\prime} \mathrm{S} 166^{\circ} 37.4^{\prime} \mathrm{E}, 330-560 \mathrm{~m}, 21$ Dec. 2006, M. Wanat leg. (MNHW); 9 specimens, ditto, track to La Tranchée, $22^{\circ} 05^{\prime} \mathrm{S} 166^{\circ} 38^{\prime} \mathrm{E}, 190-330 \mathrm{~m}, 20$ Dec. 2006, M. Wanat and R. Dobosz leg. (MNHW); 8 specimens, ditto, 21 Dec. 2006, R. Dobosz leg. (USMB); 13 specimens, ditto, 180330 m, 22-23 Jan. 2004, M. Wanat leg. (MNHW); 3 specimens, ditto, La Tranchée - Sentier des Kaoris, $22^{\circ} 05^{\prime} \mathrm{S} 166^{\circ} 38^{\prime} \mathrm{E}, 280-330 \mathrm{~m}$, humid forest, 24 Jan. 2004, M. Wanat leg. (MNHW); 8 specimens, ditto, 28 Jan. 2004 (MNHW); 2 specimens, ditto, 26 Jan. 2004 (MNHW); 1 specimen, Rivière Bleue, [-22.09833 166.63833], 10 Oct. 1978, G. Kuschel leg. (NZAC); 1 specimen, ditto, long valley, $150 \mathrm{~m}$, cooler and wetter ex Belliotum crassifolium (Baill.) van Tiegh. (Winteraceae), 18 Sep. 1976, L.B. Thien leg. (BPBM); 1 specimen, ditto (Yate), $35 \mathrm{~km}$ SE of Noumea, 160-180 m, [-22.09291 166.66431], 14 Nov. 1963, R. Straatman leg. (BPBM); 6 specimens, ditto, N of La Tranchée, $22^{\circ} 05^{\prime}$ S $166^{\circ} 38^{\prime}$ E, 280-330 m, 20 Dec. 2006, R. Dobosz and M. Wanat leg. (USMB); 2 specimens, Rivière Bleue Parc, $22^{\circ} 05.9^{\prime} \mathrm{S} 166^{\circ} 38.3^{\prime} \mathrm{E}, 190 \mathrm{~m}$, night coll. (lamp and beating), 20 Dec. 2006, M. Wanat and R. Dobosz leg. (MNHW); 2 specimens, ditto, refuge, 19 Dec. 2006 (USMB); 1 specimen, Pic du Grand Kaori, $22^{\circ} 16.8^{\prime} \mathrm{S} 166^{\circ} 53.5^{\prime} \mathrm{E}, 240 \mathrm{~m}$, night coll. (lamp and beating), 26 Dec. 2006, M. Wanat and R. Dobosz leg. (MNHW); 1 specimen, ditto, -22.28430 166.89540, 220 m, night, 1 Nov. 2010, M. Wanat and R. Ruta leg. (MNHW); 7 specimens, Bois du Sud, -22.17200 166.76111, 220 m, maquis, night beating, 20 Oct. 2008, M. Wanat leg. (MNHW); 1 specimen, ditto, at light, 23 Oct. 2008 (MNHW); 1 specimen, ditto, day beating, 18 Oct. 2008 (MNHW); 1 specimen, ditto, -22.17288 166.76330, 220-250 m, beating along track entering forest reserve, 20 Oct. 2008 (MNHW); 3 specimens, ditto, $22^{\circ} 10.5^{\prime} \mathrm{S} 166^{\circ} 45.8^{\prime} \mathrm{E}$, $160 \mathrm{~m}$, maquis, night coll. (lamp and beating), 23 Dec. 2006, M. Wanat and R. Dobosz leg. (MNHW); 1 specimen, ditto (USMB); 3 specimens, ditto, netting (USMB); 1 specimen, ditto, maquis, 24 Dec. 2006 

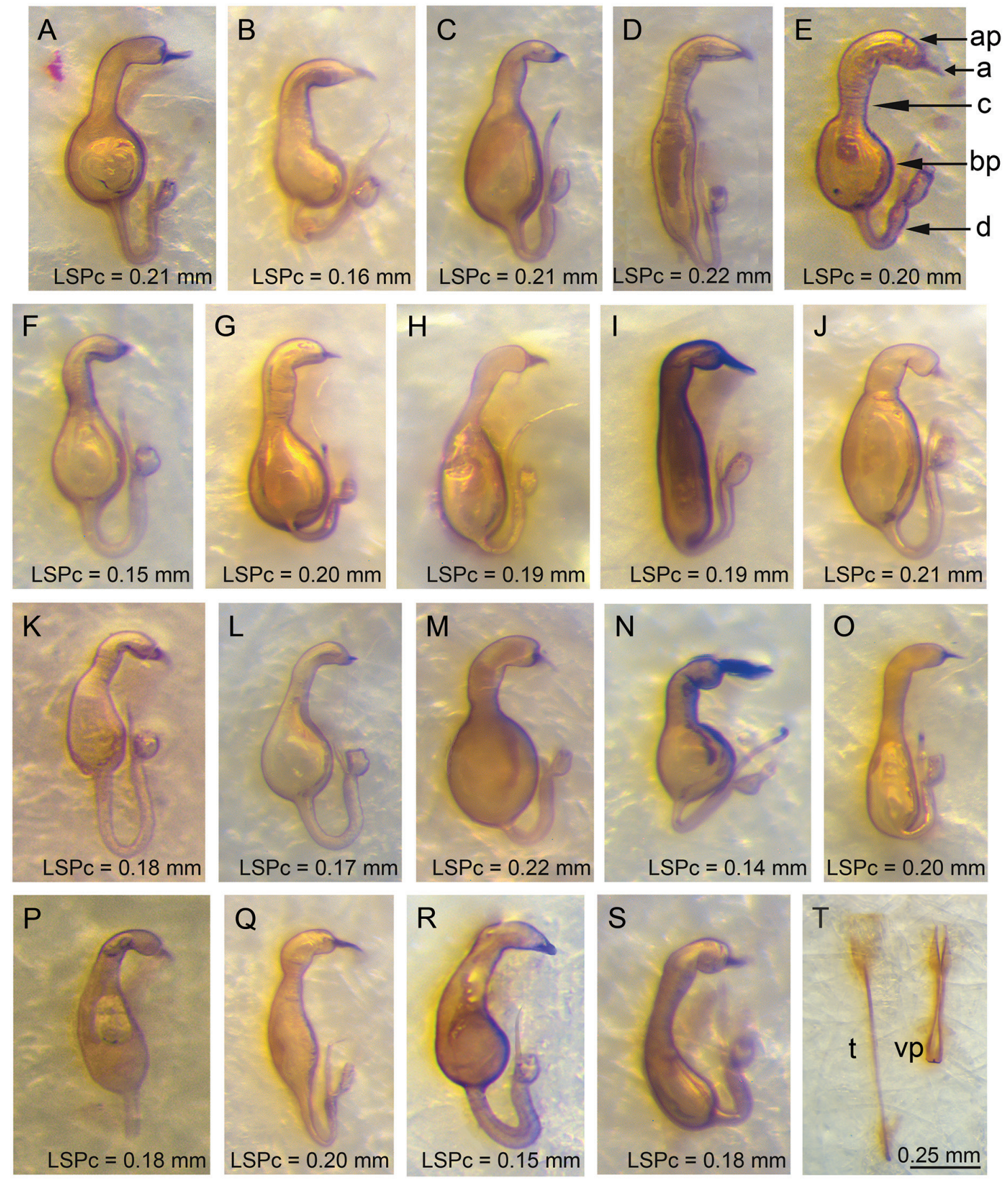

Fig. 11. Female genitalia. A-S. Spermatheca. A. Arsipoda agalma Samuelson, 1973, N of Pic Vincent. B. A. atra sp. nov., Aoupinié. C. A. communis sp. nov., Dzumac Mts. D. A. doboszi sp. nov., Mt Do. E. A.elongata sp. nov., Mt Humboldt. F. A. evax Samuelson, 1973, Chagrin. G. A. geographica GómezZurita, 2010, Mt Humboldt. H. A. gressitti sp. nov., Farino. I. A. isola Samuelson, 1973, Bois du Sud. J. A. longifrons sp. nov., Humboldt S track. K. A. paniensis sp. nov., Mt Panie. L. A. povilaensis sp. nov., Pic d'Amoa, Povila. M. A. rostrata Gómez-Zurita, 2010, Dzumac Road. N. A. rutai sp. nov. Dzumac Road. O. A. shirleyae Samuelson, 1973, Haute Rivière Bleue. P. A. transversa sp. nov., Mt Koghi. Q. A. wanati sp. nov., Mt Humboldt. R. A. yiambiae Samuelson, 1973, Yiambi. S. A. gomezzuritai sp. nov., Dzumac Mts. T. Tignum ( $\mathrm{t}$ ) and vaginal palpi (vp) of $A$. communis sp. nov., Mt Do. Abbreviations: a = appendix; $\mathrm{ap}=$ apical part; $\mathrm{bp}=$ basal part; $\mathrm{c}=$ collum or neck; $\mathrm{d}=$ ductus; $\mathrm{LSPc}=$ length of spermathecal capsule; $\mathrm{SPc}=$ spermathecal capsule $=\mathrm{ap}+\mathrm{c}+\mathrm{bp}$. 
(MNHW); 2 specimens, ditto ('Araucaria' hut), -22.17400 166.76270, 220 m, at light, 8 Dec. 2010, M. Wanat and R. Ruta leg. (MNHW); 1 specimen, ditto, at light, R. Dobosz leg. (USMB); 7 specimens, Bois du Sud, camp, $22^{\circ} 10.5^{\prime} \mathrm{S} 166^{\circ} 45.8^{\prime} \mathrm{E}, 160 \mathrm{~m}$, R. Dobosz leg. (USMB); 1 specimen, ditto, $22^{\circ} 10.443^{\prime} \mathrm{S}$ 166 45.760' E, 210 m, biting, maquis Hibbertia sp., 2 Mar. 2008 (USMB); 1 specimen, ditto, biting, netting, maquis and rainforest, 8 Mar. 2008, R. Dobosz and T. Blaik leg. (USMB); 1 specimen, Dzumac Mts, -22.02051 166.46606, 850-910 m, road from jet to old mine (km 0-1), 29 Oct. 2008, M. Wanat leg. (MNHW); 4 specimens, ditto, Mt Ouin road junction, -22.03188 166.46738, 910 m, 28 Oct. 2008

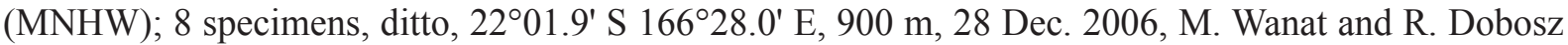
leg. (MNHW); 1 specimen, ditto, R. Dobosz leg. (USMB); 1 specimen, ditto, netting, 30 Dec. 2006 (USMB); 3 specimens, ditto (base), km 1.5-3 E Ouin rd jet [= road junction], -22.03710 166.49570, 800 m, rainforest, 6 Dec. 2010, R. Ruta and M. Wanat leg. (MNHW); 2 specimens, Dzumac Rd, km 0-1 $\mathrm{E}$ of Ouin $\mathrm{rd}$ jct [= road junction], $-22.03340166 .47480,800-900 \mathrm{~m}$, to refuge, maquis, 6 Dec. 2010, M. Wanat and R. Ruta leg. (MNHW); 1 specimen, Mouirange Pass [-22.21689 166.65008], 10 Feb. 1962, N.L.H. Krauss leg. (BPBM); 1 specimen, Col de Mouirange [-22.233333 166.649722], 2 Feb. 1963, C.M. Yoshimoto leg. (BPBM); 2 specimens, ditto (forest patch $1 \mathrm{~km} \mathrm{E} \mathrm{of),} \mathrm{[-22.21350} \mathrm{166.66550],}$ 240 m, 9 Dec. 2010, M. Wanat and R. Ruta leg. (MNHW); 23 specimens, 30 km NW Col de Mouirange [-22.210252 166.689641], $175 \mathrm{~m}$, on Gmelina neocaledonica young plants leaves w/mines [= with mines], 10 Aug. 1979, G.A. Samuelson leg. (BPBM); 2 specimens, Chute de la Madeleine, 22 ${ }^{\circ} 14^{\prime} \mathrm{S}$ $166^{\circ} 52^{\prime}$ E, 270 m, maquis, 13 Feb. 2004, M. Wanat leg. (MNHW); 3 specimens, ditto, $22^{\circ} 14.2^{\prime} \mathrm{S}$ $166^{\circ} 51.7^{\prime}$ E, 240 m, maquis, night coll. (lamp and beating), 24 Dec. 2006, M. Wanat and R. Dobosz leg. (MNHW); 2 specimens, ditto (USMB); 3 specimens, ditto, 240-270 m, maquis, 25 Dec. 2006 (MNHW); 2 specimens, Koghi Mts, [-22.17631 166.50138], 340 m, roadside, 25 Oct. 2008, M. Wanat leg. (MNHW); 3 specimens, ditto, [-22.16643 166.53365], 420 m, on ferns, 9 Aug. 1979, G.A. Samuelson leg. (BPBM); 1 specimen, ditto, [-22.17302 166.51730], 600-900 m, 19 Mar. 1968, T.C. Maa leg. (BPBM); 1 specimen, ditto, [-22.18333 166.50000], 400-600 m, Jan. 1969, N.L.H. Krauss leg. (BPBM); 3 specimens, ditto, summit [-22.16144 166.51296], 18 Mar. 1968, J.L. Gressitt and T.C. Maa leg. (BPBM); 1 specimen, track Col des Agathis to Forest Cachee, -22.17288 166.76330, 500-340 m, 26 Oct. 2008, M. Wanat leg. (MNHW); 4 specimens, Col des Deux Tétons, -22.20587 166.67967, 220 250 m, forest, 4 Dec. 2008, M. Wanat leg. (MNHW); 2 specimens, ditto, -22.20590 166.67970, 220-250 m, humid forest, 9 Dec. 2010, M. Wanat and R. Ruta leg. (MNHW); 1 specimen, ditto, humid forest, at light (MNHW); 2 specimens, Mt Mou, 1000-1200 m, [-22.06168 166.34922], 9 Mar. 1972, J.L. Gressitt leg. (BPBM); 1 specimen, ditto, [-22.06612 166.35169], $800 \mathrm{~m}$ (BPBM); 1 specimen, ditto, [-22.07711 166.35466], 400 m, 7 Mar. 1972 (BPBM); 1 specimen, ditto, [-22.07287 166.35444], 500-800 m, 2 Feb. 1978, J.S. Dugdale leg. (BPBM); 3 specimens, between Plum and Yate [-22.16975 166.75320], 25 Mar. 1968, T.C. Maa leg. (BPBM); 1 specimen, Yate, 5 km ex Fork, 2-50 m, 25 Mar. 1968, J.L. Gressitt and T.C. Maa leg. (BPBM); 1 specimen, Mt Ouénarou, [-22.16667 166.71667], 300 m, 24 Oct. 1978, G. Kuschel leg. (NZAC); 1 specimen, Les Pirogues [Pirogues Bay], [-22.30449 166.67938], 8 Oct. 1978, G. Kuschel leg. (NZAC); 10 specimens, Valle de Thy, [-22.18330 166.53300], 250-500 m, 28-29 Mar. 1981, J.L. Gressitt and G.M. McPherson leg. (BPBM).

\section{Taxonomic notes}

A. shirleyae is, along with A. geographica and A. gressitti sp. nov., one of the yellowish species lacking external morphological characters that allow a reliable identification. These species can be distinguished by relying on the morphology of genitalia (Figs 8H-I, 9I, 11G-H, O). Median lobe of aedeagus (Fig. 9I) in ventral view thickset, laterally subparallel, with narrower base; apical part widened, laterally rounded, with large median tooth; ventral sulcus V-shaped, clearly impressed median groove; in lateral view median lobe distinctly curved, with slightly sinuate dorsal and ventral sides; dorsal ligula from apical third to almost apex of median lobe of aedeagus, basally widened, then clearly narrower. Spermatheca (Fig. 11O) with pyriform basal part; collum very elongate (as long as basal part); length of apical part about half as long as collum; ductus short, apically inserted. 


\section{Biometry}

Male ( $\mathrm{n}=10$; mean and standard deviation; range): $\mathrm{LE}=1.60 \pm 0.09 \mathrm{~mm}(1.43 \leq \mathrm{LE} \leq 1.73 \mathrm{~mm}) ; \mathrm{WE}=$ $1.03 \pm 0.06 \mathrm{~mm}(0.93 \leq \mathrm{WE} \leq 1.13 \mathrm{~mm}) ; \mathrm{LP}=0.50 \pm 0.03 \mathrm{~mm}(0.45 \leq \mathrm{LP} \leq 0.55 \mathrm{~mm}) ; \mathrm{WP}=0.76 \pm$ $0.04 \mathrm{~mm}(0.71 \leq \mathrm{WP} \leq 0.81 \mathrm{~mm}) ; \mathrm{LAN}=1.35 \pm 0.06 \mathrm{~mm}(1.23 \leq \mathrm{LAN} \leq 1.45 \mathrm{~mm}) ; \mathrm{LAED}=0.92 \pm$ $0.03 \mathrm{~mm}(0.86 \leq \mathrm{LAED} \leq 0.95 \mathrm{~mm}) ; \mathrm{LB}=2.29 \pm 0.11 \mathrm{~mm}(2.13 \leq \mathrm{LB} \leq 2.44 \mathrm{~mm}) ; \mathrm{LE} / \mathrm{LP}=3.19 \pm 0.11$ $(3.00 \leq \mathrm{LE} / \mathrm{LP} \leq 3.30) ; \mathrm{WE} / \mathrm{WP}=1.36 \pm 0.04(1.31 \leq \mathrm{WE} / \mathrm{WP} \leq 1.43) ; \mathrm{WP} / \mathrm{LP}=1.51 \pm 0.04(1.45 \leq \mathrm{WP} /$ $\mathrm{LP} \leq 1.58) ; \mathrm{WE} / \mathrm{LE}=0.64 \pm 0.01(0.62 \leq \mathrm{WE} / \mathrm{LE} \leq 0.66) ; \mathrm{LAN} / \mathrm{LB}=0.59 \pm 0.02(0.55 \leq \mathrm{LAN} / \mathrm{LB} \leq$ $0.62) ; \mathrm{LE} / \mathrm{LAED}=1.74 \pm 0.06(1.66 \leq \mathrm{LE} / \mathrm{LAED} \leq 1.84)$.

Female ( $\mathrm{n}=10$; mean and standard deviation; range): $\mathrm{LE}=1.77 \pm 0.10 \mathrm{~mm}(1.63 \leq \mathrm{LE} \leq 1.95 \mathrm{~mm})$; $\mathrm{WE}=1.12 \pm 0.07 \mathrm{~mm}(1.00 \leq \mathrm{WE} \leq 1.23 \mathrm{~mm}) ; \mathrm{LP}=0.54 \pm 0.03 \mathrm{~mm}(0.50 \leq \mathrm{LP} \leq 0.58 \mathrm{~mm}) ; \mathrm{WP}=$ $0.82 \pm 0.05 \mathrm{~mm}(0.75 \leq \mathrm{WP} \leq 0.91 \mathrm{~mm}) ; \mathrm{LAN}=1.28 \pm 0.7 \mathrm{~mm}(1.20 \leq \mathrm{LAN} \leq 1.43 \mathrm{~mm}) ; \mathrm{LSPc}=0.21 \pm$ $0.01 \mathrm{~mm}(0.20 \leq \mathrm{LSPc} \leq 0.22 \mathrm{~mm}) ; \mathrm{LB}=2.48 \pm 0.14 \mathrm{~mm}(2.22 \leq \mathrm{LB} \leq 2.69 \mathrm{~mm}) ; \mathrm{LE} / \mathrm{LP}=3.27 \pm 0.08$
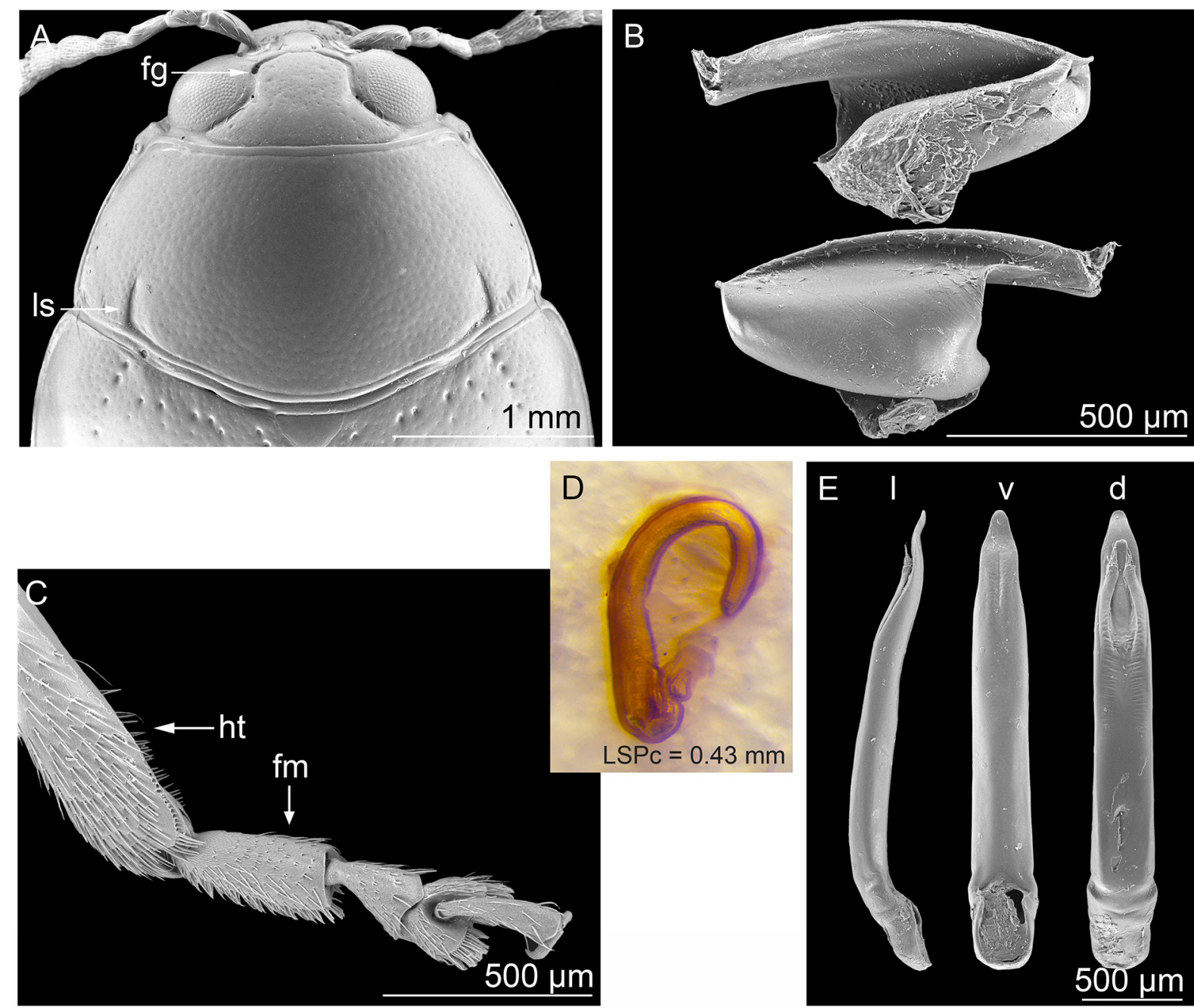

Fig. 12. Morphological traits of Arsipoda bifrons Erichson, 1973. A. Head and pronotum. B. Metafemoral spring. C. Hind tibia and tarsus. D. Spermatheca. E. Median lobe of aedeagus in lateral (1), ventral (v) and dorsal (d) views. A-C, E: Australia (BAQ); D: Australia, Byron Bay (BAQ). Abbreviations: $\mathrm{fg}=$ frontal grooves; $\mathrm{fm}=$ first metatarsomere; $\mathrm{ht}=$ hind tibia; $1 \mathrm{~s}=$ longitudinal striae; $\mathrm{LSPc}=$ length of spermathecal capsule. 
$(3.09 \leq \mathrm{LE} / \mathrm{LP} \leq 3.39) ; \mathrm{WE} / \mathrm{WP}=1.37 \pm 0.03(1.33 \leq \mathrm{WE} / \mathrm{WP} \leq 1.42) ; \mathrm{WP} / \mathrm{LP}=1.51 \pm 0.05(1.43 \leq \mathrm{WP} /$ $\mathrm{LP} \leq 1.59) ; \mathrm{WE} / \mathrm{LE}=0.63 \pm 0.01(0.62 \leq \mathrm{WE} / \mathrm{LE} \leq 0.66) ; \mathrm{LAN} / \mathrm{LB}=0.52 \pm 0.02(0.49 \leq \mathrm{LAN} / \mathrm{LB} \leq$ $0.54) ; \mathrm{LE} / \mathrm{LSPc}=8.53 \pm 0.45(7.81 \leq \mathrm{LE} / \mathrm{LSPc} \leq 9.31)$.

\section{Distribution}

Central and most of Southern Grande Terre (Fig. 15A).

\section{Ecological notes}

Collected in plantation, camp, maquis, dry scrub, forest edge, moss forest, humid forest, rainforest, from 50 to $1200 \mathrm{~m}$ a.s.l. Found on Neoguillauminia sp. (Euphorbiaceae), Belliotum crassifolium (Winteraceae), Araucaria sp. (Auracariaceae); Hibbertia sp. (Dilleniaceae), and Gmelina neocaledonica (Lamiaceae). Pollen found in the gut of some specimens.

\section{Arsipoda transversa sp. nov. \\ urn:1sid:zoobank.org:act:1B5665C9-3AE0-4644-8427-A4C3E997B4BD}

Figs 2I, 5D, 10A, 11P, 15A

\section{Diagnosis}

Arsipoda transversa sp. nov. shares a thickset, ovoid body with A. atra sp. nov., A. evax and A. povilaensis sp. nov. (Figs 1B, F, 2D, I). It can be distinguished by the more transverse and more convex pronotum, and the generally reddish integuments, sometimes with darker head and pronotum (A. atra sp. nov. is black, A. evax is black and metallic, and A. povilaensis sp. nov. is yellowish). Genitalia are also clearly distinguishable (Figs 8C, G, 9E, 10A, 11B, F, L, P).

\section{Etymology}

The name is a Latinized adjective referring to the distinctively transverse pronotum of the species.

\section{Type material}

\section{Holotype}

NEW CALEDONIA: đ̊, Mt Koghi, 400-600 m, [-22.17833 166.50667], Feb. 1973, N.L.H. Krauss leg. (BPBM).

\section{Paratypes}

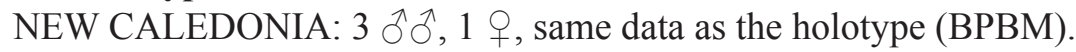

\section{Description of the holotype $(\stackrel{\text { đ) }}{)}$}

Body thickset, moderately convex (Fig. 2I); LB $=2.23 \mathrm{~mm}$; maximum pronotal width at base $(\mathrm{WP}=0.94 \mathrm{~mm})$; maximum elytral width at basal third $(\mathrm{WE}=1.17 \mathrm{~mm})$. Dorsum light brown, non-metallic. Frons and vertex very finely microreticulate and sparsely punctate; head grooves weakly impressed, not continued to postantennal region; genae and frontal carina short; antennae slightly longer than $1 / 2$ body length $(\mathrm{LAN}=1.30 \mathrm{~mm}$; $\mathrm{LAN} / \mathrm{LB}=0.58)$, basally yellow, gradually and slightly darker toward apex; LA:100:82:54:73:82:91:100:91:91:91:136.

Pronotum trapezoidal, clearly transverse ( $\mathrm{LP} 0.50 \mathrm{~mm}$; WP/LP $=1.88$ ), laterally straight (Fig. $5 \mathrm{D}$ ); anterior angles distinctly prominent; antebasal transverse sulcus shallow, slightly sinuate, with visible lateral fovea; pronotal surface finely microreticulate, with very small and dense punctuation. Elytra moderately elongate $(\mathrm{LE}=1.76 \mathrm{~mm} ; \mathrm{WE} / \mathrm{LE}=0.66)$, laterally clearly arcuate; punctuation quite small, but distinctly larger than on pronotum; interstriae flat, very finely microreticulate. Anterior and middle legs and hind tarsi yellow, hind femora and tibiae light brown; first pro- and mesotarsomeres moderately dilated; adhesive setae present on 
ventral side of first pro-, meso- and, partially, metatarsomeres. Venter light brown; last abdominal ventrite laterally clearly incised, without special preapical impressions. Median lobe of aedeagus (LAED $=0.92 \mathrm{~mm}$; LE/LAED = 1.91) (Fig. 10A) in ventral view laterally parallel at basal half, then slightly tapered; apex triangular with rounded tip; ventral sulcus complete, wide and deep for most of its length; in lateral view median lobe bent at basal third, then straight; dorsal ligula from half length to apical part of median lobe of aedeagus, narrow.

\section{Biometry}

Male $(\mathrm{n}=3$; range): $1.54 \leq \mathrm{LE} \leq 1.76 \mathrm{~mm} ; 1.02 \leq \mathrm{WE} \leq 1.17 \mathrm{~mm} ; 0.42 \leq \mathrm{LP} \leq 0.50 \mathrm{~mm} ; 0.78 \leq \mathrm{WP} \leq$ $0.94 \mathrm{~mm} ; 1.20 \leq \mathrm{LAN} \leq 1.30 \mathrm{~mm} ; 0.87 \leq \mathrm{LAED} \leq 0.92 \mathrm{~mm} ; 1.95 \leq \mathrm{LB} \leq 2.23 \mathrm{~mm} ; 3.52 \leq \mathrm{LE} / \mathrm{LP} \leq$ $3.67 ; 1.24 \leq \mathrm{WE} / \mathrm{WP} \leq 1.31 ; 1.83 \leq \mathrm{WP} / \mathrm{LP} \leq 1.88 ; 0.65 \leq \mathrm{WE} / \mathrm{LE} \leq 0.66 ; 0.58 \leq \mathrm{LAN} / \mathrm{LB} \leq 0.62 ; 1.76 \leq$ $\mathrm{LE} / \mathrm{LAED} \leq 1.91$.
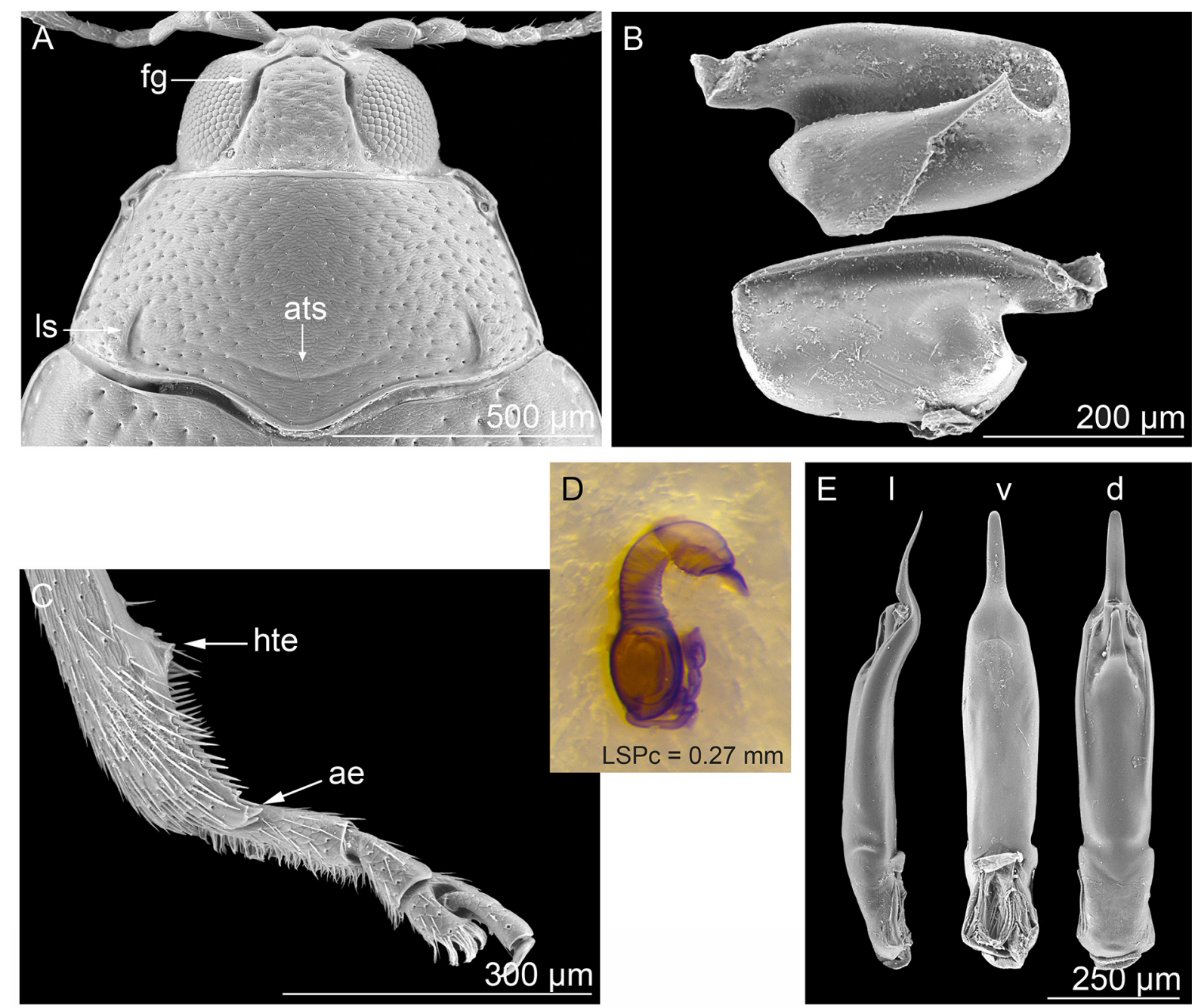

Fig. 13. Morphological traits of Crepicnema parvula (Jacoby, 1885) comb. nov., Solomon Islands, San Cristobal (BMNH). A. Head and pronotum. B. Metafemoral spring. C. Hind tibia and tarsus. D. Spermatheca. E. Median lobe of aedeagus in lateral (l), ventral (v) and dorsal (d) views. Abbreviations: ae $=$ apical emargination with thickset spines; ats $=$ antebasal transverse sulcus; hte $=$ emargination on outer margin of hind tibia; ls = longitudinal striae; LSPc = length of spermathecal capsule; $t \mathrm{fg}=$ frontal grooves. 
Female $(\mathrm{n}=1): \mathrm{LE}=1.84 \mathrm{~mm} ; \mathrm{WE}=1.24 \mathrm{~mm} ; \mathrm{LP}=0.52 \mathrm{~mm} ; \mathrm{WP}=0.96 \mathrm{~mm} ; \mathrm{LAN}=1.22 \mathrm{~mm}$; $\mathrm{LSPc}=0.18 \mathrm{~mm} ; \mathrm{LB}=2.35 \mathrm{~mm} ; \mathrm{LE} / \mathrm{LP}=3.54 ; \mathrm{WE} / \mathrm{WP}=1.29 ; \mathrm{WP} / \mathrm{LP}=1.85 ; \mathrm{WE} / \mathrm{LE}=0.67 ; \mathrm{LAN} /$ $\mathrm{LB}=0.52 ; \mathrm{LE} / \mathrm{LSPc}=10.00$.

Paratypes very similar in shape sculpture, and color to the holotype. One specimen with darker head and pronotum. Spermatheca (Fig. 11P) with pear-shaped basal part; collum about twice as long as apical part, thickset, clearly tapered toward apical part; ductus thickset, apically inserted (distal part missing).

\section{Distribution}

Endemic to Mt Koghi, Southern Grande Terre (Fig. 15A).

\section{Ecological notes}

No information is available about habitat and host plants. Pollen found in the gut of some specimens.

$$
\begin{aligned}
& \text { Arsipoda wanati sp. nov. } \\
& \text { urn:1sid:zoobank.org:act:5DFBCA5B-E85B-4C20-98A0-D3804D0FCB30 }
\end{aligned}
$$

Figs 3A, 5E, 10B, 11Q, 15B

\section{Diagnosis}

Among the yellowish species, $A$. wanati sp. nov. can be easily identified by the bicolored antennae, with antennomeres 1-4 yellow and 5-11 brown and by the blackish maxillary and labial palpi, distinctly darker than other mouth parts.

\section{Etymology}

Male patronym. Named after Dr. Marek Wanat (University of Wroclaw, Poland), one of its collectors.

\section{Type material}

\section{Holotype}

NEW CALEDONIA (S): đa, Mt Humboldt, -21.87930 166.42320, 1500-1618 m, montane maquis, 13 Nov. 2010, M. Wanat \& R. Ruta leg. (MNHW).

\section{Paratypes}

NEW CALEDONIA: $6 \hat{\jmath} \widehat{\jmath}, 2$ 우, same data as the holotype (MNHW); $5 \hat{\jmath} \widehat{\partial}, 3$ 웅, ditto, ca $1400 \mathrm{~m}$ (USMB); 1 , ditto, -21.88120 166.41770, 1400-1500 m (MNHW).

\section{Description of the holotype $(ふ)$}

Body moderately elongate and convex (Fig. $3 \mathrm{~A}$ ); $\mathrm{LB}=2.66 \mathrm{~mm}$; maximum pronotal width at base $(\mathrm{WP}=0.98 \mathrm{~mm})$; maximum elytral width at basal third $(\mathrm{WE}=1.33 \mathrm{~mm})$. Dorsum yellowish, non-metallic, with suture and lateral margin of elytra darkened. Frons and vertex microreticulate, with small punctures distinctly impressed; head grooves distinctly impressed, not continued to postantennal region; genae and frontal carina short; maxillary and labial palpi blackish, distinctly darker than other mouth parts; third and fourth maxillary palpomeres flat; antennae clearly longer than half of body length $(\mathrm{LAN}=1.93 \mathrm{~mm}$; LAN/LB $=0.72)$; antennomeres $1-4$ yellow, 5-11 brown; LA: 100:59:59:6 5:100:88:100:91:88:85:115. Pronotum trapezoidal, clearly transverse ( $\mathrm{LP}=0.53 \mathrm{~mm}$; WP/LP $=1.86$ ), laterally straight (Fig. 5E); anterior angles weakly prominent; antebasal transverse sulcus moderately impressed, slightly sinuate, with distinct punctures on basal side; lateral fovea small; pronotal surface finely microreticulate, with small, shallow punctuation. Elytra moderately elongate $(\mathrm{LE}=2.03 \mathrm{~mm}$; $\mathrm{WE} / \mathrm{LE}=0.65$ ), laterally slightly arcuate; punctuation quite small but clearly larger and more strongly impressed than on pronotum; interstriae very finely microreticulate and sparsely micropunctuate. Legs 
yellow, with third to fifth tarsomeres darkened; first pro- and mesotarsomeres slightly dilated; adhesive setae present on ventral side of first pro-, meso- and, partially, metatarsomeres. Venter light brown; last abdominal ventrite laterally incised, without special preapical impressions. Median lobe of aedeagus $(\mathrm{LAED}=1.08 \mathrm{~mm} ; \mathrm{LE} / \mathrm{LAED}=1.88)(\mathrm{Fig} .10 \mathrm{~B})$ in ventral view laterally straight, apically widely and regularly rounded; ventral surface with deep median groove, variable in length; in lateral view, median lobe distinctly curved, sinuate at apical third; dorsal ligula short, from apical third to sub-apical part of median lobe of aedeagus, wide, slightly tapered toward apex.

\section{Biometry}

Male ( $\mathrm{n}=10$; mean and standard deviation; range): $\mathrm{LE}=2.08 \pm 0.09 \mathrm{~mm}(1.98 \leq \mathrm{LE} \leq 2.23 \mathrm{~mm}) ; \mathrm{WE}=$ $1.39 \pm 0.06 \mathrm{~mm}(1.30 \leq \mathrm{WE} \leq 1.45 \mathrm{~mm}) ; \mathrm{LP}=0.55 \pm 0.03 \mathrm{~mm}(0.53 \leq \mathrm{LP} \leq 0.60 \mathrm{~mm}) ; \mathrm{WP}=0.98 \pm$ $0.05 \mathrm{~mm}(0.93 \leq \mathrm{WP} \leq 1.06 \mathrm{~mm}) ; \mathrm{LAN}=1.94 \pm 0.10 \mathrm{~mm}(1.83 \leq \mathrm{LAN} \leq 2.13 \mathrm{~mm}) ; \mathrm{LAED}=1.11 \pm$ $0.03 \mathrm{~mm}(1.06 \leq \mathrm{LAED} \leq 1.17 \mathrm{~mm}) ; \mathrm{LB}=2.82 \pm 0.14 \mathrm{~mm}(2.66 \leq \mathrm{LB} \leq 3.06 \mathrm{~mm}) ; \mathrm{LE} / \mathrm{LP}=3.75 \pm 0.10$ $(3.60 \leq \mathrm{LE} / \mathrm{LP} \leq 3.87) ; \mathrm{WE} / \mathrm{WP}=1.42 \pm 0.05(1.36 \leq \mathrm{WE} / \mathrm{WP} \leq 1.54) ; \mathrm{WP} / \mathrm{LP}=1.76 \pm 0.05(1.69 \leq \mathrm{WP} /$ $\mathrm{LP} \leq 1.86) ; \mathrm{WE} / \mathrm{LE}=0.67 \pm 0.02(0.65 \leq \mathrm{WE} / \mathrm{LE} \leq 0.71) ; \mathrm{LAN} / \mathrm{LB}=0.69 \pm 0.02(0.66 \leq \mathrm{LAN} / \mathrm{LB} \leq$ $0.72) ; \mathrm{LE} / \mathrm{LAED}=1.88 \pm 0.06(1.80 \leq \mathrm{LE} / \mathrm{LAED} \leq 1.96)$.

Female ( $\mathrm{n}=6$; mean and standard deviation; range): $\mathrm{LE}=2.31 \pm 0.07 \mathrm{~mm}(2.24 \leq \mathrm{LE} \leq 2.43 \mathrm{~mm}) ; \mathrm{WE}=$ $1.55 \pm 0.07 \mathrm{~mm}(1.48 \leq \mathrm{WE} \leq 1.65 \mathrm{~mm}) ; \mathrm{LP}=0.60 \pm 0.02 \mathrm{~mm}(0.58 \leq \mathrm{LP} \leq 0.63 \mathrm{~mm}) ; \mathrm{WP}=1.07 \pm$ $0.04 \mathrm{~mm}(1.03 \leq \mathrm{WP} \leq 1.13 \mathrm{~mm}) ; \mathrm{LAN}=1.82 \pm 0.08 \mathrm{~mm}(1.70 \leq \mathrm{LAN} \leq 1.90 \mathrm{~mm}) ; \mathrm{LSPc}=0.19 \pm$ $0.01 \mathrm{~mm}(0.18 \leq \mathrm{LSPc} \leq 0.20 \mathrm{~mm}) ; \mathrm{LB}=3.06 \pm 0.14 \mathrm{~mm}(2.88 \leq \mathrm{LB} \leq 3.25 \mathrm{~mm}) ; \mathrm{LE} / \mathrm{LP}=3.82 \pm 0.09$ $(3.73 \leq \mathrm{LE} / \mathrm{LP} \leq 3.96) ; \mathrm{WE} / \mathrm{WP}=1.45 \pm 0.05(1.40 \leq \mathrm{WE} / \mathrm{WP} \leq 1.54) ; \mathrm{WP} / \mathrm{LP}=1.78 \pm 0.04(1.71 \leq \mathrm{WP} /$ $\mathrm{LP} \leq 1.80) ; \mathrm{WE} / \mathrm{LE}=0.67 \pm 0.02(0.65 \leq \mathrm{WE} / \mathrm{LE} \leq 0.70) ; \mathrm{LAN} / \mathrm{LB}=0.60 \pm 0.02(0.57 \leq \mathrm{LAN} / \mathrm{LB} \leq$ $0.63) ; \mathrm{LE} / \mathrm{LSPc}=11.87 \pm 0.72(11.25 \leq \mathrm{LE} / \mathrm{LSPc} \leq 13.18)$.

Paratypes generally very similar in shape, sculpture and color to the holotype. Spermatheca with fusiform basal part (Fig. 11Q); collum and apical part short; ductus elongate, thin, apically inserted, proximally not clearly separated from basal part.

\section{Distribution}

Endemic to Mt Humboldt, Southern Grande Terre (Fig. 15B).

\section{Ecological notes}

Collected in montane maquis between 1400 and $1600 \mathrm{~m}$ a.s.l. No information is available about host plants.

Arsipoda yiambiae Samuelson

Figs 3B, 5F, 10C, 11R, 15A

Arsipoda yiambiae Samuelson, 1973: 132.

Arsipoda yiambiae - Gómez-Zurita et al. 2010: 2560.

\section{Type material examined}

NEW CALEDONIA: holotype, ô, Yiambi, NE, [-20,47008 164,70042], 500-700 m, 14 Oct. 1967 (BPBM).

\section{Taxonomic notes}

Arsipoda yiambiae and A. paniensis sp. nov. are the only species with a pronotum clearly convex laterally (Figs 4F, 5F), but they are clearly different in color and size: A. paniensis sp. nov. is dark green, metallic, 
and larger; A. yiambiae is yellow and smaller. Genitalia are also easily distinguishable (Figs 9D, 10C, $11 \mathrm{~K}, \mathrm{R}$ ). Median lobe of aedeagus slender (Fig. 10C); parallel in ventral view, distinctly enlarged at basal third, with triangular apical part; ventral sulcus thin and shallow; in lateral view median lobe curved at basal third, then straight; dorsal ligula narrow, fusiform. Spermatheca (Fig. 11R) with rounded basal part; collum very elongate (as long as basal part); length of apical part about half as long as collum; ductus elongate, thickset, apically inserted.

\section{Biometry}

Male (holotype): $\mathrm{LE}=1.65 \mathrm{~mm} ; \mathrm{WE}=1.04 \mathrm{~mm} ; \mathrm{LP}=0.48 \mathrm{~mm} ; \mathrm{WP}=0.82 \mathrm{~mm} ; \mathrm{LAN}=1.12 \mathrm{~mm}$; $\mathrm{LAED}=1.05 \mathrm{~mm} ; \mathrm{LB}=2.15 \mathrm{~mm} ; \mathrm{LE} / \mathrm{LP}=3.44 ; \mathrm{WE} / \mathrm{WP}=1.27 ; \mathrm{WP} / \mathrm{LP}=1.71 ; \mathrm{WE} / \mathrm{LE}=0.63 ; \mathrm{LAN} /$ $\mathrm{LB}=0.52 ; \mathrm{LE} / \mathrm{LAED}=1.58$.

\section{Distribution}

Collected in Northern and Central Grande Terre (Fig. 15A).

\section{Ecology}

It is increasingly evident that New Caledonian Arsipoda can utilize a broad range of plant associates (some as possible larval food plants) and that pollen-feeding is included in adult feeding strategies (Gomez-Zurita et al. 2010; Samuelson 1989, 1994).

We report plant associate data for eight species of Arsipoda and of these, seven are associated with more than one botanical plant family. A. isola, so far, shows the greatest range with 9 families. In one collection of $A$. isola taken on leaves of Gmelina neocaledonica (Lamiaceae), beetles were aggregating on fresh leaves of new plants and these leaves included many active larval mines (observed by collector). These reported plant associates belong to 14 orders and 16 families according to APG III Classification System (Bremer et al. 2009): Anacardiaceae (Sapindales); Aquifoliales (Aquifoliaceae); Auracariaceae (Pinales); Cunoniaceae (Oxalidales); Cyperaceae (Poales); Dilleniaceae (incert order in APGIII System (Bremer et al. 2009)); Ericaceae, Primulaceae - now including Myrsinaceae -, Symplocaceae (Ericales); Euphorbiaceae (Malpighiales); Fabaceae (Fabales); Lamiaceae (Lamiales); Myrtaceae (Myrtales); Pandanaceae (Pandanales); Proteaceae (Proteales); Winteraceae (Canellales).

Pollen grains were found in the gut of 13 species of either sex, including species lacking plant identifications. It is likely that most species of New Caledonian Arsipoda will be shown to eat pollen. Mouthparts adapted for pollen uptake may show special modifications: e.g., long and dense setae on the mandible inner lobe and outer margin, the maxillary galea and lacinia, and flat areas at the base of the mandible for grinding pollen grains (Bieńkowski 2010; Samuelson 1994). Mouthparts of A. shirleyae (Fig. 7A-B) have long setae on the maxillae, but not on the mandibles, which have an inner lobe covered by short and dense setae that may be used for pollen feeding; this species also lacks mandibular grinding areas and that may explain the intact pollen grains in the gut. The mode of pollen digestion by these beetles remains unknown (Bieńkowski 2010; Samuelson 1994).

\section{Taxonomy}

Within the variability of Arsipoda from all areas, the New Caledonian species show a high degree of similarity (Figs 1A-I, 2A-I, 3A-C). They generally possess the following features: 1) small size; 2) frontal tubercles absent (Figs 4A-F, 5A-F);3) frontal grooves short, generally not continued anteriorly to postantennal region (exceptions in A. agalma) (Figs 4A-F, 5A-F); 4) antennomeres 1-2 quite more globose than antennomere $3 ; 5$ ) pronotal antebasal transverse sulcus generally present, variable from weakly to distinctly impressed, laterally bounded by small dimples, sometimes elongate, but neither clearly impressed nor clearly reaching the basal margin (Figs 4A-F, 5A-F); 6) pronotal lateral margin 
generally straight or concave (Figs 4A-E, 5A-E), distinctly convex only in A. paniensis sp. nov. and A. yiambiae (Figs 4F, 5F); 7) inner edge of hind tibiae with an apical emargination with thickset spines (Fig. 6E); 8) hind tarsi thin; 9) first metatarsomeres sub-rectangular, about as long as the remaining four together (Fig. 6E); 10) metafemoral spring of the Psylliodes morpho-group (Furth \& Suzuki 1998) (Fig. 6F); 11) spermathecal morphology of Type A (Furth \& Suzuki 1994, 1998) (Figs 11A-S). These character states are different in A. bifrons Erichson from Australia (Figs 12A-E), type species of the genus, which shows: 1) larger size; 2) frontal tubercles present; 3 ) frontal grooves clearly continued anteriorly to the postantennal region; 4) antennomeres $1-2$ not globose; 5) pronotum lacking the antebasal transverse sulcus, but with latero-basal longitudinal striae clearly impressed and reaching the basal margin; 6) lateral margin of pronotum distinctly convex; 7) inner edge of hind tibiae without any apical emargination; 8) hind tarsi more thickset; 9) first metatarsomeres sub-conical, shorter than the remaining four together; 10) metafemoral spring probably belonging to the Altica morpho-group (Furth \& Suzuki 1998); 11) spermathecal morphology of atypical Type A though not of Type B-Galerucinae type (Furth \& Suzuki 1994, 1998).

Crepicnema Scherer, 1969 (Figs 13A-E) is a monotypic genus including the only species C. parvula (Jacoby, 1885) comb. nov. (= Chaetocnema tenimberensis Jacoby, 1894 syn. nov. = Arsipoda salomonensis Bryant, 1941 syn. nov.) widespread in Oriental and Australian regions (Blackburn 1896; Bryant 1937; Heikertinger \& Csiki 1940; Gruev 1982; Jacoby 1896; Medvedev 1993; Medvedev \& Romantsov 2013; Samuelson 1967; Scherer 1969; Weise 1917). It shares character states 2, 4, 5, 6, 7, 8, 9, 10 with New Caledonian Arsipoda (noted in the numeration above), and is obviously a close relative. It is further distinguished from Arsipoda by the presence of a distinct emargination on the outer margin of the hind tibiae (Fig. 13C) (Biondi 2002; Scherer 1969).

\section{Phylogenetic analysis}

To test the relationships among the New Caledonian species, we included as outgroups Arsipoda bifrons, as a "typical" large species, and Crepicnema. Our parsimony analysis used 34 bi-state or tristate morphological characters (see Appendix A). The strict consensus tree is reported in Fig. $16(\mathrm{~K}=$ 2.66; Consistency Index $(\mathrm{CI})=0.449$; Retention Index $(\mathrm{RI})=0.488)$.

The New Caledonian species of Arsipoda are arranged in the tree as a monophyletic group supported by a high jackknife value (87) (Fig. 16). Even though it is likely that they represent a natural group derived from a possible isolated radiation, we need a phylogenetic analysis based on a broader dataset to assess their actual monophyly. The position of A. agalma is notable due to its presence of a deep frontal groove running to the upper ocular margin (Character 1 in Appendix A): the only character state also present in the two outgroup species and not shared by A. agalma with its New Caledonian congeners.

A significant matter arising from this tree concerns the taxonomic position of the genera Arsipoda and Crepicnema. Three alternative hypotheses can be proposed: a) the New Caledonian species of Arsipoda are to be attributed to the genus Crepicnema; b) the New Caledonian species of Arsipoda are to attribute to a new flea beetle genus; c) the genus Crepicnema has to be considered a synonym of Arsipoda. For now we prefer not to take any decision about these three possible hypotheses, leaving any definitive interpretation until a suitable and more complete phylogenetic dataset will become available and the taxonomic situation of the Arsipoda species from other geographic areas will be clearer.

\section{Distribution}

Most of the New Caledonian Arsipoda species are restricted to Grande Terre, the main island. Only A. evax is known from a nearby island (Belep Island), cited by Gómez-Zurita et al. (2010). Thus far, we have no records of Arsipoda from the Isle of Pines or the Loyalty Islands. Based on our data, the 

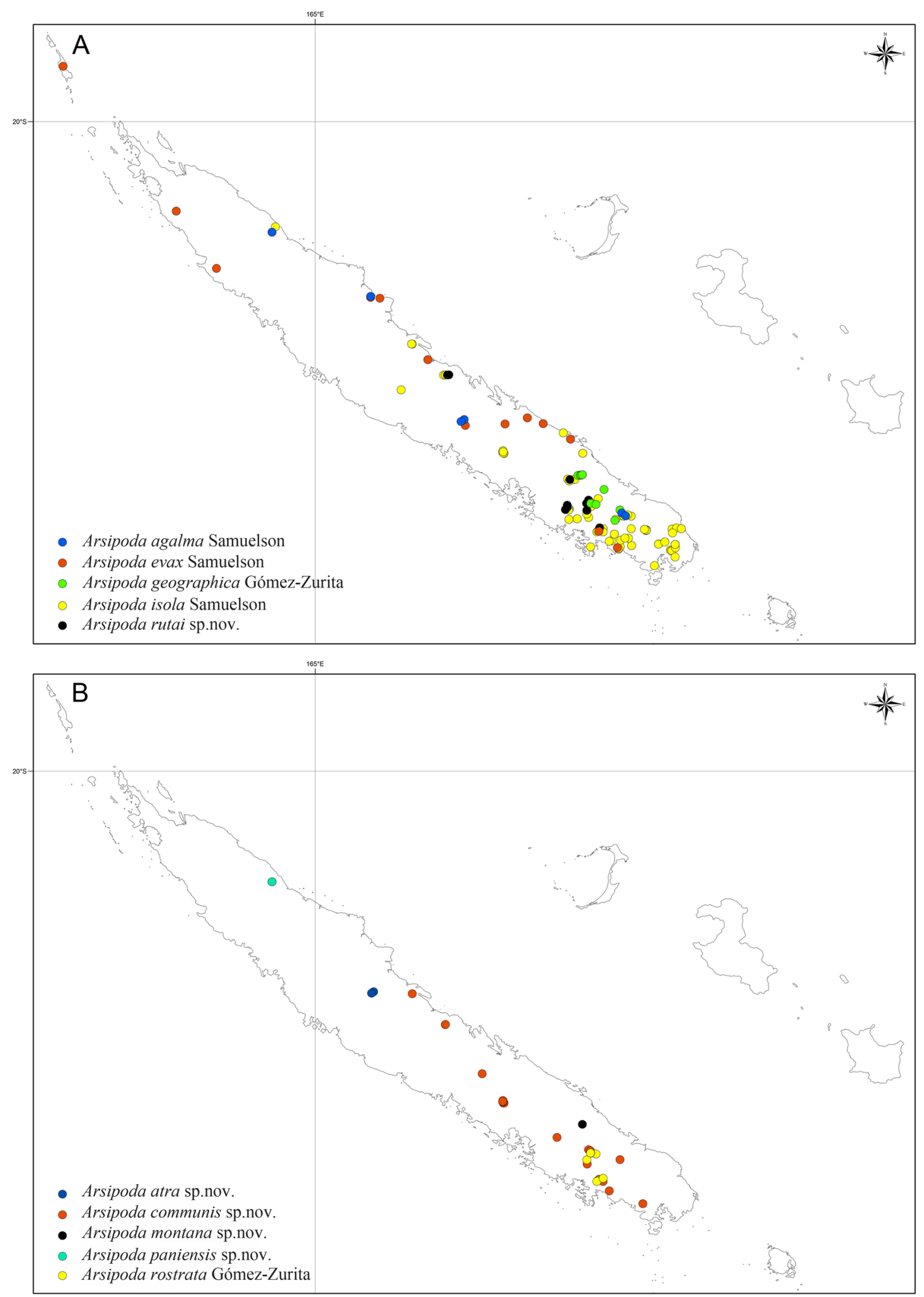

Fig. 14. Distributions of New Caledonian Arsipoda. A. Arsipoda agalma Samuelson, 1973, A. evax Samuelson, 1973, A. geographica Gómez-Zurita, 2010, A. isola Samuelson, 1973 and A. rutai sp. nov. B. A. atra sp. nov., A. communis sp. nov., A. montana sp. nov., A. paniensis sp. nov. and A. rostrata Gómez-Zurita, 2010. 

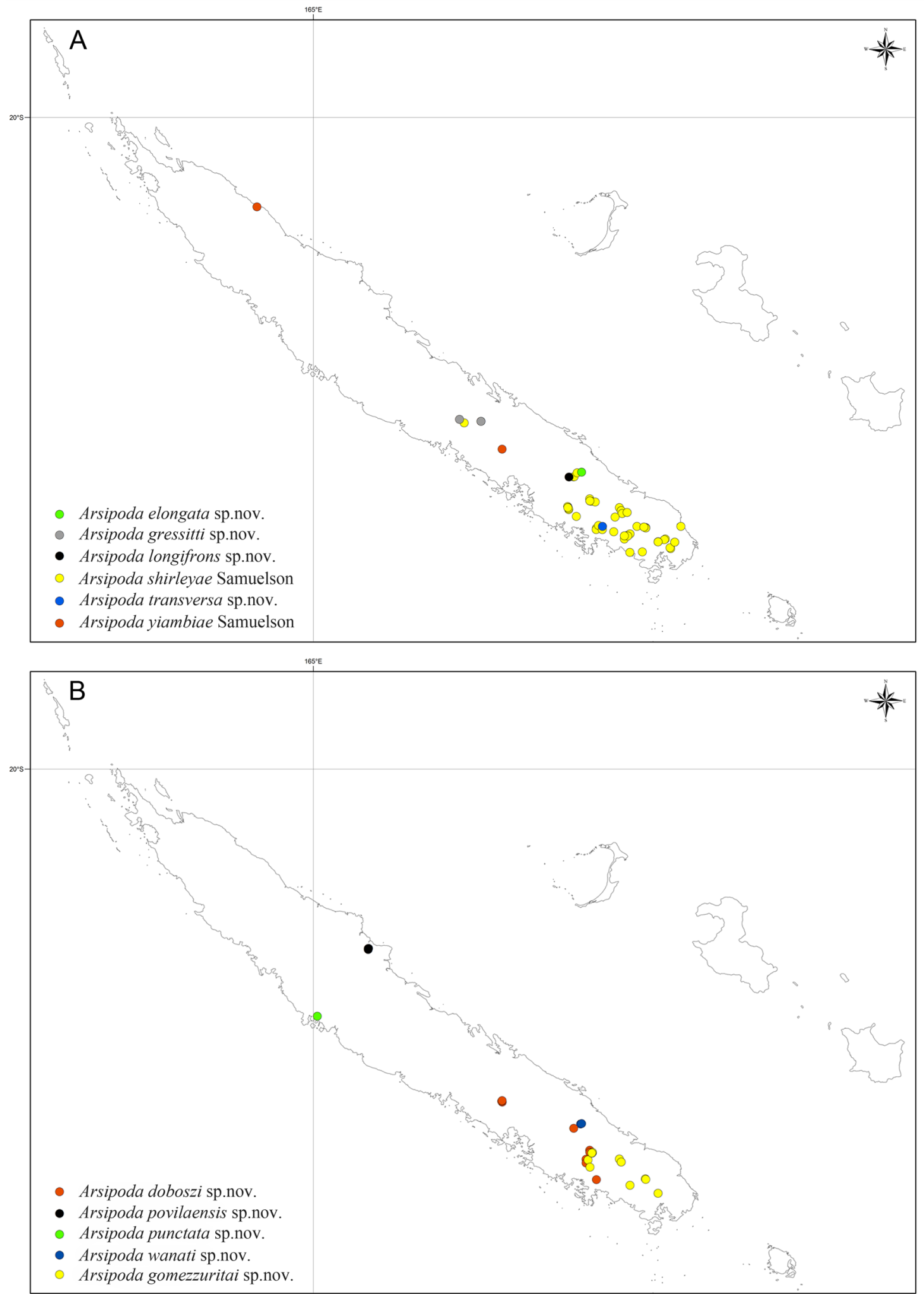

Fig. 15. Distributions of New Caledonian Arsipoda. A. Arsipoda elongata sp. nov., A. gressitti sp. nov., A. longifrons sp. nov., A. shirleyae Samuelson, 1973, A. transversa sp. nov. and A. yiambiae Samuelson, 1973. B. A. doboszi sp. nov., A. povilaensis sp. nov., A. punctata sp. nov., A. wanati sp. nov. and A. gomezzuritai sp. nov. 
distributions of the species in New Caledonia vary from more or less widespread, even though often rare, to strictly microendemic, with a major number of species and a denser distribution in the Southern part of the island (Figs 14A-B, 15A-B). We have to point out, however, that in New Caledonia there are some logistic and cultural differences which make it easier to collect in the South Province; thus, the distribution map may reflect a bias in the sampling activity (Gómez-Zurita pers. comm.). Nevertheless, some trends in the distribution patterns of the New Caledonian fauna (Pellens \& Grandcolas 2010) can be deduced from these data: a) individual species are generally rare; b) short range endemism appears more frequently as distributional studies accumulate; c) sister species apparently appeared by allopatric speciation and are often separated by very short distances, even though some with a more disjunct distribution have been considered as relicts in refuge areas. In our analysis, one of the three significantly supported clades (Fig. 16) within the New Caledonian Arsipoda, [rostrata (gomezzuritai, longifrons)], links species all endemic to the South Province with partially overlapping or slightly isolated distributions (Figs 14B, 15A-B). In this clade, we can recognize a pattern of endemism of the type II of Pellens \& Grandcolas (2010): short range endemic sister species with parapatric/allopatric distributions. The same situation can be observed for A. geographica and A. shirleyae in the clade [geographica, punctata, shirleyae], even though the clade is unresolved and $A$. shirleyae has a slightly wider distribution; $A$. punctata sp. nov. appears rather isolated from the other two species. A parapatric distribution can be also seen between $A$. yiambiae and $A$. rutai sp. nov. and between $A$. rutai sp. nov. and A. transversa sp. nov. within the unresolved clade [rutai, transversa, yiambiae]; in this case, A. yiambiae and A. rutai sp. nov. display a relatively wide distribution rather than a "short ranged" distribution

The clade [wanati (paniensis, povilaensis)] is different, in that it includes allopatric species attributable to the pattern of endemism of type III of Pellens \& Grandcolas (2010), characterized by related species with disjunct distributions occurring also in the North Province.

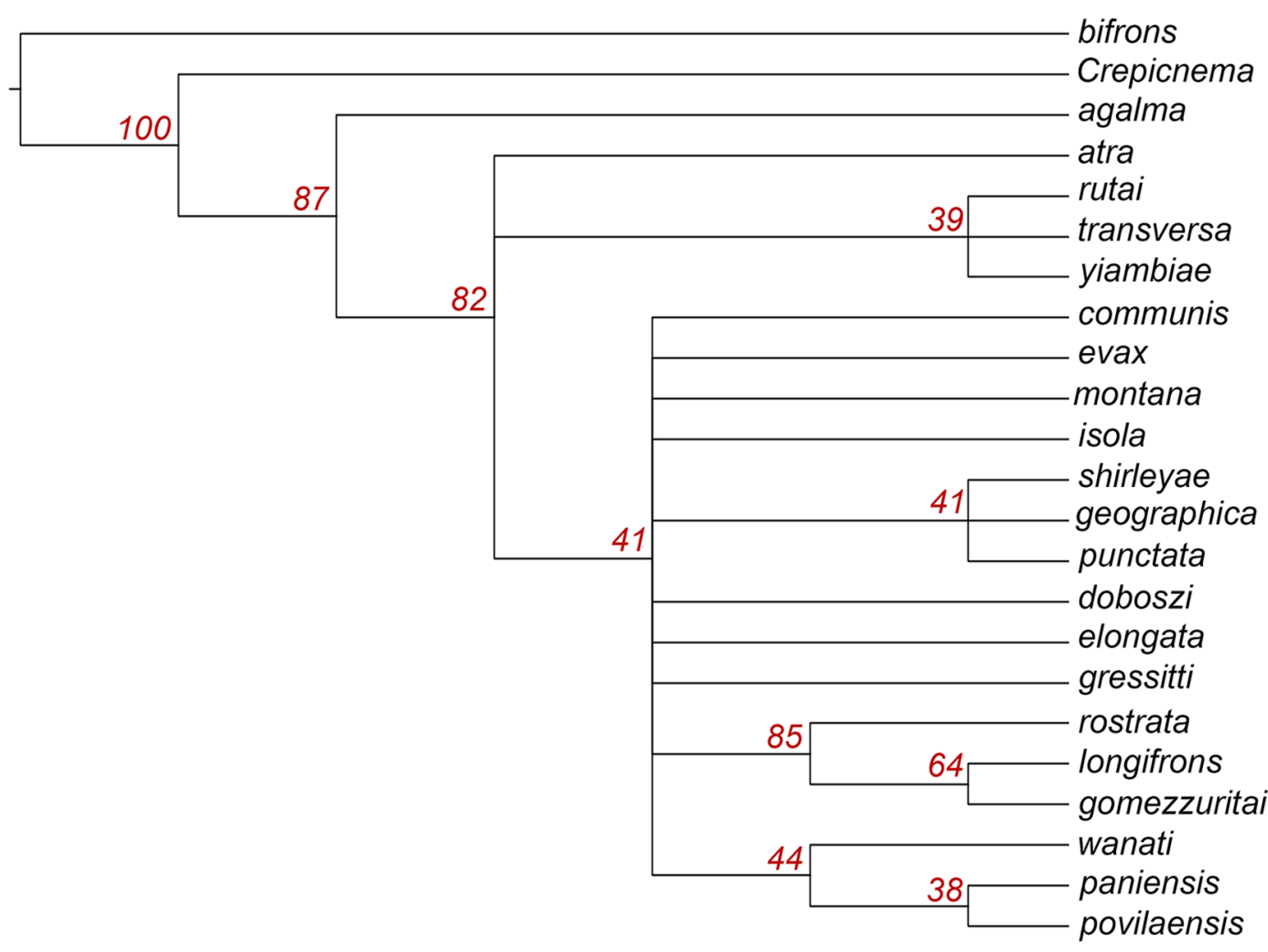

Fig. 16. Strict consensus tree with jackknife values (see text). 


\section{Discussion}

Our study, based on more than 1550 specimens of Arsipoda, tripled the number of known species of New Caledonia from seven to 21, but may, nevertheless, still be regarded as preliminary. All these species are macropterous, and they are potentially able to move easily; also they seem broadly adaptable regarding food choice. Yet, some of these species are microendemic, being restricted to limited areas. These traits accordingly invite further collecting, especially in hard to reach locations, and they also create a need for general studies of their habits. So far, only one species of Arsipoda has been associated with an exotic host. Arsipoda evax was collected on flowers of mango (Mangifera indica), denoting a shift to a new host from native hosts of several diverse families. That event must have occurred quite recently, noting that most Mangifera introductions to Pacific islands took place only over the past 100 years (Bally 2006). Additional species and data on their ecologies will certainly come to light, as further surveys are conducted in the New Caledonia area, including the Loyalty Islands. Furthermore, expanding Arsipodacentered studies to surrounding areas will also help to clarify whether the New Caledonian elements are truly monophyletic, and perhaps help to clarify the status between Arsipoda and Crepicnema.

\section{Acknowledgements}

We are grateful to all colleagues and friends who enabled us to study material in their respective institutions. Special thanks go to James Boone (BPBM), Michael Geiser (BMNH), Antoine Mantilleri (MNHN), Roberto Poggi (MSNG) and Marek Wanat (MNHW). We are also thankful to Dr. Jesús GómezZurita (Institut de Biologia Evolutiva, Barcelona, Spain) for his suggestions to improve this paper.

\section{References}

AA.VV. 2011. Delineation of Key Biodiversity Areas. New Caledonia. The New Caledonia Ecosystem Profile Working group.

Bally I.S. 2006. Mangifera indica (mango). In: C.R. Elevitch (ed.) Traditional Trees of Pacific Islands. Their Culture, Environment, and Use: 441-464. Permanent Agriculture Resources, Holualoa, Hawaii.

Beenen R. 2008. Contribution to the knowledge of Galerucinae of New Caledonia (Coleoptera: Chrysomelidae). Genus 19 (1): 65-87.

Beenen R. 2013. Contribution to the knowledge of Galerucinae of New Caledonia 2 (Coleoptera: Chrysomelidae). Genus 24 (1): 65-108.

Bieńkowski A.O. 2010. Anthophagy in the Leaf Beetles (Coleoptera, Chrysomelidae). Entomological Review 90 (4): 423-432. http://dx.doi.org/10.1134/S0013873810040032

Biondi M. 2002. Chaetocnema Stephens and related genera (Coleoptera, Chrysomelidae, Alticinae): a systematic revision, with description of a new genus from the Indian Ocean (Seychelles). Italian Journal of Zoology 69 (4): 355-366. http://dx.doi.org/10.1080/11250000209356481

Biondi M. \& D'Alessandro P. 2012. Afrotropical flea beetle genera: a key to their identification, update catalogue and biogeographical analysis (Coleoptera, Chrysomelidae, Galerucinae, Alticini). Zookeys 253: 1-158. http://dx.doi.org/10.3897/zookeys.253.3414

Blackburn T. 1896. Further notes on Australian Coleoptera, with descriptions of new genera and species. Part XIX. Transactions of the Royal Society of South Australia 20: 35-109.

Bouchard P., Bousquet Y., Davies A.E., Alonso-Zarazaga M.A., Lawrence J.F., Lyal C.H.C., Newton A.F., Reid C.A.M., Schmitt M., Ślipiński S.A. \& Smith A.B.T. 2011. Family-group names in Coleoptera (Insecta). ZooKeys 88: 1-972. http://dx.doi.org/10.3897/zookeys.88.807 
Bremer B., Bremer K., Chase M.W., Fay M.F., Reveal J.L., Soltis D.E., Soltis P.S. \& Stevens P.F. 2009. An update of the Angiosperm Phylogeny Group classification for the orders and families of flowering plants: APG III. Botanical Journal of the Linnean Society 161 (2): 105-121. http://dx.doi.org/10.1111/ j.1095-8339.2009.00996.x

Bryant G.E. 1937. Notes on synonymy in the Phytophaga (Coleoptera). Annals and Magazine of Natural History series 1020 (115): 97-101. http://dx.doi.org/10.1080/00222933708655320

Bryant G.E. 1941. New species of Chrysomelidae (Coleoptera) from New Guinea, Solomon Is. and Fiji. Annals and Magazine of Natural History series 118 (44): 96-105. http://dx.doi.org/10.1080/03745481 .1941 .9727957

Chapuis F. 1875. Famille LXIX. Phytophages (suite). Tribu XIII. Galérucides. In: Lacordaire T. \& Chapuis F. (eds) Histoire Naturelle des Insectes. Genera des Coléoptères. Tome XI. Librairie Encyclopédique de Roret, Paris.

Clark H. 1864. Descriptions of new Australian Phytophaga. Journal of Entomology 2 (11): 247-263.

D'Alessandro P., Urbani F. \& Biondi M. 2014. Biodiversity and biogeography in Madagascar: revision of the endemic flea beetle genus Neodera Duvivier, 1891 with description of 19 new species (Coleoptera, Chrysomelidae, Galerucinae, Alticini). Systematic Entomology 39 (4): 710-748. http:// dx.doi.org/10.1111/syen.12082

Döberl M. 1986. Die Spermathek als Bestimmungshilfe bei den Alticinen. Entomologische Blätter 82: $3-14$.

Erichson W.F. 1842. Beitrag zur Insecten-fauna von Vandiemensland, mit besonderer Berücksichtigung der geographischen Verbreitung der Insecten. Archiv für Naturgeschichte 8 (1): 83-287. http://dx.doi. org/10.5962/bhl.part.21656

Espeland M. \& Murienne J. 2011. Diversity dynamics in New Caledonia: towards the end of the museum model? BMC Evolutionary Biology 11: 254. http://dx.doi.org/10.1186/1471-2148-11-254

Furth D.G. 1982. The Metafemoral Spring of Flea Beetles (Chrysomelidae: Alticinae). Spixiana 7: 11-27.

Furth D.G. \& Suzuki K. 1994. Character correlation studies of problematic genera of Alticinae in relation to Galerucinae (Coleoptera: Chrysomelidae). In: D.G. Furth (ed.) Proceedings of the Third International Symposium on the Chrysomelidae, Beijing, 1992: 116-135. Backhuys Publishers, Leiden.

Furth D.G. \& Suzuki K. 1998. Studies of Oriental and Australian Alticinae genera based on the comparative morphology of the metafemoral spring, genitalia, and hind wing venation. In: Biondi M., Daccordi M. \& Furth D.G. (eds) Proceedings of the Fourth International Symposium on the Chrysomelidae. Proceedings of XX I.C.E, Firenze, 1996: 91-124. Museo Regionale di Scienze Naturali, Torino.

Gasc A., Sueur J., Pavoine S., Pellens R. \& Grandcolas P. 2013. Biodiversity sampling using a global acoustic approach: contrasting sites with microendemics in New Caledonia. Plos ONE 8 (5): e65311. http://dx.doi.org/10.1371/journal.pone.0065311

Gillespie R.G. \& Roderick G.K. 2002. Arthropods on islands: Colonization, speciation, and conservation. Annual Review of Entomology 47: 595-632. http://dx.doi.org/10.1146/annurev.ento.47.091201.145244

Goloboff P., Carpenter J., Arias S. \& Miranda D. 2008a. Weighting against homoplasy improves phylogenetic analysis of morphological data sets. Cladistics 24: 758-773. http://dx.doi.org/10.1111/ j.1096-0031.2008.00209.x

Goloboff P., Farris J. \& Nixon K. 2008b. TNT: a free program for phylogenetic analysis. Cladistics 24: 774-786. http://dx.doi.org/10.1111/j.1096-0031.2008.00217.x 
Goloboff P. 2013. Extended implied weighting. Cladistics 30 (3): 260-272. http://dx.doi.org/10.1111/ cla. 12047

Gómez-Zurita J., Cardoso A., Jurado-Rivera J.A., Jolivet P., Cazères S. \& Mille C. 2010. Discovery of new species of New Caledonian Arsipoda Erichson, 1842 (Coleoptera: Chrysomelidae) and insights on their ecology and evolution using DNA markers. Journal of Natural History, 44 (41-42): 2557-2579. http://dx.doi.org/10.1080/00222933.2010.499575

Gómez-Zurita J. 2011. Rhyparida foaensis (Jolivet, Verma \& Mille, 2007), comb. n. (Coleoptera, Chrysomelidae) and implications for the colonization of New Caledonia. ZooKeys 157: 33-44. http:// dx.doi.org/10.3897/zookeys.157.1320

Gómez-Zurita J. \& Cardoso A. 2013. Systematics of the New Caledonian endemic genus Taophila Heller (Coleoptera: Chrysomelidae, Eumolpinae) combining morphological, molecular and ecological data, with description of two new species. Systematic Entomology 39 (1): 111-126. http://dx.doi.org/10.1111/ syen. 12038

Grandcolas P., Murienne J., Robillard T., Desutter-Grandcolas L., Jourdan H., Guilbert E. \& Deharveng L. 2008. New Caledonia: a very old Darwinian island? Philosophical Transactions of the Royal Society, Series B 363: 3309-3317. http://dx.doi.org/10.1098/rstb.2008.0122

Gruev B. 1982. Neue Angaben über einige Blattkäfer aus der Alten Welt. Faunistische Abhandlungen Staatliches Museum für Tierkunde in Dresden 9 (8): 109-114.

Heikertinger F. \& Csiki E. 1940. Chrysomelidae: Halticinae. In: Schenkling S. (ed.) Coleopterorum catalogus auspiciis et auxilio. Parts 166 and 169. Junk, The Hague.

Jacoby M. 1885. Descriptions of new genera and species of phytophagous Coleoptera from the IndoMalayan and Austro-Malayan subregions, contained in the Genoa Civic Museum. Second part. Annali del Museo Civico di Storia Naturale Genova 22: 20-76.

Jacoby M. 1894. Description of new genera and species of phytophagous Coleoptera obtained by W. Doherty in the Malayan Archipelago. Novitates Zoologicae 1 (2): 267-330. http://dx.doi.org/10.5962/ bhl.part.24565

Jacoby M. 1896. Descriptions of the new genera and species of Phytophagous Coleoptera obtained by Dr. Modigliani in Sumatra. Annali del Museo Civico di Storia Naturale di Genova, serie 216 (36): 377-501. http://dx.doi.org/10.5962/bhl.part.25039

Jolivet P. \& Verma K.K. 2008. On the origin of the Chrysomelid Fauna of New Caledonia. In: Jolivet P., Santiago-Blay J. \& Schmitt M. (eds) Research on Chrysomelidae. Vol. 1: 309-319. Brill, Leiden.

Jolivet P. \& Verma K.K. 2009. Biogeography and biology of the New Caledonian Chrysomelidae (Coleoptera). In: Jolivet P., Santiago-Blay J. \& Schmitt M. (eds) Research on Chrysomelidae. Vol. 2: 211-223. Brill, Leiden. http://dx.doi.org/10.1163/ej.9789004169470.1-299.83

Jolivet P. \& Verma K.K. 2010. Good morning Gondwana. Annales de la Société entomologique de France (N.S.) 46 (1-2): 53-61. http://dx.doi.org/10.1080/00379271.2010.10697638

Kier G., Kreft H., Lee T.M., Jetz W., Ibisch P.L., Nowicki C., Mutke J. \& Barthlott W. 2009. A global assessment of endemism and species richness across island and mainland regions. Proceedings of the National Academy of Sciences of the United States of America 106 (23): 9322-9327. http://dx.doi. org/10.1073/pnas.0810306106

Kimoto S., Ismay J.W. \& Samuelson G.A. 1984. Distribution of Chrysomelid Pests Associated with Certain Agricultural Plants in Papua New Guinea (Coleoptera). Esakia 21: 49-57. 
Lowry P.P. II, Munzinger J., Bouchet P., Géraux H., Bauer A.M., Langrand O. \& Mittermeier R.A. 2004. New Caledonia. In: Mittermeier R.A., Robles Gil P., Hoffmann M., Pilgrim J., Brooks T., Mittermeier C.G., Lamoreux J. L. \& da Fonseca G.A.B. (eds) Hotspots Revisited: Earth's Biologically Richest and Most Threatened Terrestrial Ecoregions: 193-197. Cemex, Mexico City.

Medvedev L.N. 1993. Alticinae of the Philippine Islands (Coleoptera Chrysomelidae). Part 1. Russian Entomological Journal 2 (3-4): 41-58.

Medvedev L.N. \& Romantsov P.V. 2013. New and poorly known Chrysomelidae (Coleoptera) from South-East Asia. Caucasian Entomological Bulletin 9 (1): 137-140.

Mirande J.M. 2009. Weighted parsimony phylogeny of the family Characidae (Teleostei: Characiformes). Cladistics 25 (6): 574-613. http://dx.doi.org/10.1111/j.1096-0031.2009.00262.x

Mittermeier R.A., Robles Gil P., Hoffmann M., Pilgrim J., Brooks T., Mittermeier C.G., Lamoreux J.L. \& da Fonseca G.A.B. (eds) 2004. Hotspots Revisited: Earth's Biologically Richest and Most Threatened Terrestrial Ecoregions. Cemex, Mexico City.

Mohamedsaid 2004. Catalogue Of The Malaysian Chrysomelidae (Insecta: Coleoptera). Series Faunistica 36, Pensoft Publishers, Sofia, Bulgaria.

Myers N., Mittermeier R.A., Mittermeier C.G., da Fonseca G.A.B. \& Kent J. 2000. Biodiversity hotspots for conservation priorities. Nature 403: 853-858. http://dx.doi.org/10.1038/35002501

Myers N. 2003. Biodiversity hotspots revisited. BioScience 53 (10): 916-917. http://dx.doi.org/ $\underline{10.1641 / 0006-3568(2003) 053[0916: B H R] 2.0 . C O ; 2}$

Nattier R., Grandcolas P., Elias M., Desutter-Grandcolas L., Jourdan H., Couloux A. \& Robillard T. 2012. Secondary Sympatry Caused by Range Expansion Informs on the Dynamics of Microendemism in a Biodiversity Hotspot. PLOS ONE 7 (11): e48047. http://dx.doi.org/10.1371/journal.pone.0048047

Nattier R., Grandcolas P., Pellens R., Jourdan H., Couloux A., Poulain S. \& Robillard T. 2013. Climate and Soil Type Together Explain the Distribution of Microendemic Species in a Biodiversity Hotspot. PLOS ONE 8 (12): e80811. http://dx.doi.org/10.1371/journal.pone.0080811

Papadopoulou A., Cardoso A. \& Gómez-Zurita J. 2013. Diversity and diversification of Eumolpinae (Coleoptera: Chrysomelidae) in New Caledonia. Zoological Journal of the Linnean Society 168 (3): 473495. http://dx.doi.org/10.1111/zoj.12039

Pellens R. \& Grandcolas P. 2010. Conservation and management of the biodiversity in a hotspot characterized by short range endemism and rarity: the challenge of New Caledonia. In: Rescigno V. \& Maletta S. (eds) Biodiversity Hotspot: 139-151. Nova Science Publishers, Hauppauge, New York.

Possingham H.P. \& Wilson KA. 2005. Biodiversity: Turning up the heat on hotspots. Nature 436: 919 920. http://dx.doi.org/10.1038/436919a

Samuelson 1967. Alticinae of the Solomon Islands (Coleoptera: Chrysomelidae). Pacific Insects 9 (1): 139-174.

Samuelson G.A. 1973. Alticinae of Oceania (Coleoptera: Chrysomelidae). Pacific Insects Monograph 30, Entomology Dept., Bernice P. Bishop Museum, Honolulu.

Samuelson G.A. 1989. Pollen feeding in Alticinae (Chrysomelidae). Entomography 6: 407-411.

Samuelson G.A. 1994. Pollen consumption and digestion by leaf beetles. In: Jolivet P.H., Cox M.L. \& Petitpierre E. (eds) Novel aspects of the biology of Chrysomelidae: 179-183. Kluwer Academic Publisher, Dordrecht. http://dx.doi.org/10.1007/978-94-011-1781-4_10 
Samuelson G.A. 2010. Review of Taophila, a genus endemic to New Caledonia (Coleoptera: Chrysomelidae: Eumolpinae). Zootaxa 2621: 45-62.

Scherer G. 1969. Die Alticinae des Indischen Subkontinentes (Coleoptera - Chrysomelidae). Pacific Insects Monograph 22, Entomology Dept., Bernice P. Bishop Museum, Honolulu.

Seeno T.N. \& Wilcox J.A. 1982. Leaf beetle genera (Coleoptera: Chrysomelidae). Entomography 1, Entomography Publications, Sacramento.

Slipinski S.A., Leschen R.A.B., \& Lawrence J. F. 2011. Order Coleoptera Linnaeus, 1758. In: Zhang Z.Q. (ed.) Animal biodiversity: An outline of higher-level classification and survey of taxonomic richness. Zootaxa 3148: 203-208.

Suzuki K. 1988. Comparative morphology of the internal reproductive system of the Chrysomelidae (Coleoptera). In: Jolivet P., Petitpierre E. \& Hsiao T.H. (eds) Biology of Chrysomelidae: 317-355. Series Entomologica 42. Kluwer Academic, Dordrecht. http://dx.doi.org/10.1007/978-94-009-3105-3 19

Weise J. 1917. Chrysomelidae. In: Nova Guinea. Vol. V. Uitkomsten der Nederlandsche Nieuw-GuineaExpeditie in 1903 Onder Leiding van Dr. Arthur Wichmann: 311-349. Brill, Leiden.

Zhang Z.-Q. 2011. Phylum Arthropoda von Siebold, 1848. In: Zhang Z.-Q. (ed.) Animal biodiversity: An outline of higher-level classification and survey of taxonomic richness. Zootaxa 3148: 99-103.

Manuscript received: 23 November 2015

Manuscript accepted: 27 February 2016

Published on: 15 September 2016

Topic editor: Koen Martens

Desk editor: Charlotte Thionois

Printed versions of all papers are also deposited in the libraries of the institutes that are members of the EJT consortium: Muséum national d'Histoire naturelle, Paris, France; Botanic Garden Meise, Belgium; Royal Museum for Central Africa, Tervuren, Belgium; Natural History Museum, London, United Kingdom; Royal Belgian Institute of Natural Sciences, Brussels, Belgium; Natural History Museum of Denmark, Copenhagen, Denmark; Naturalis Biodiversity Center, Leiden, the Netherlands. 
Appendix A. List of the morphological characters and state-characters used for the parsimony analysis (matrix in the supplementary material)

\section{Head}

1. Deep suprantennal furrow running to the upper ocular margin: present (0); absent (1)

2. Anterior part of vertex: convex (0); concave or flat (1)

3. Frontal tubercles: present (0); absent (1)

4. Antennomere 10: about as long as antennomere $1(0)$; longer than antennomere 1, at least in male (1)

5. Antennomeres 1-2: not more globose than antennomere $3(0)$; more globose than antennomere 3 (1)

6. Eyes: in frontal view laterally prominent (0); in frontal view laterally not prominent (1)

\section{Pronotum}

7. Pronotal lateral margin: convex (0); straight or concave (1)

8. Pronotal antebasal transversal sulcus: absent (0); present (shallow, partially obliterate or clearly impressed) (1)

9. Pronotal longitudinal striae: elongate and clearly reaching the basal margin (0); dimples not reaching the basal margin (1)

\section{Ventral parts}

10. Prosternum: smooth, or sparsely or marginally punctate only (0); strongly and evenly punctate (1)

11. Prosternal antecoxal space (a): as wide as intercoxal process (0); wider than intercoxal process (1)

12. Prosternal antecoxal space (b): clearly transversally carinate (0); flat or almost flat (1)

13. Median impression on last abdominal segment in male: absent (0); present (1)

\section{Legs}

14. Emargination on outer side of hind tibiae: absent (0); present (1)

15. Apical emargination with spines on inner edge of hind tibiae: absent (0); present (1)

16. First metatarsomere (a): conical (0); cylindrical (1)

17. First metatarsomere (b): shorter than the remaining four together (0); about as long as the remaining four together (1)

18. Metafemoral spring: slender (0); thickset (1)

\section{Spermatheca}

19. Spermathecal capsule: without clearly distinguishable basal and distal parts (0); with clearly distinguishable basal and distal parts (1)

20. Spermathecal basal part: elongate (at least twice its width) (0); short (shorter than twice its width) (1)

21. Spermathecal collum: basally not narrowed (0); basally narrowed (1)

22. Spermathecal appendix: absent (0); present (1)

23. Spermathecal ductus (a): uncoiled (0); coiled (1)

24. Spermathecal ductus (b): thickset (0); thin (1)

25. Spermathecal ductus (c): proximally very close to capsule (0); proximally detached from capsule (1)

\section{Median lobe of aedeagus}

26. Median lobe of aedeagus in ventral view: laterally parallel (0); laterally fusiform or tapered towards apex (1); tapered towards base (2)

27. Ventral sulcus of median lobe of aedeagus: present (at least shallow, thin) (0); absent (1)

28. Apical part of median lobe of aedeagus: not wider than base (0); distinctly wider than base (1)

29. Apical part of median lobe of aedeagus: acute (0); rounded/obtuse (1)

30. Apical tooth or tooth-like extension: present (0); absent (1) 
31. Pre-apical part of median lobe of aedeagus: narrowed (0); not narowed (1)

32. Dorsal ligula of median lobe of aedeagus: sub-parallel or tapered towards apex (0); fusiform (1)

33. Dorsal ligula length: base within the apical third of the median lobe of aedeagus (0); base more towards the base of the median lobe of aedeagus (1)

34. Dorsal ligula width: base narrower than $1 / 2$ of the median lobe of aedeagus width (0); base wider than $1 / 2$ of the median lobe of aedeagus width (1) 